\title{
Power indices, claims games and core selection
}

Citation for published version (APA):

Kong, Q. (2021). Power indices, claims games and core selection. [Doctoral Thesis, Maastricht University, Northwestern Polytechnical University]. Maastricht University. https://doi.org/10.26481/dis.20210208qk

Document status and date:

Published: 01/01/2021

DOI:

10.26481/dis.20210208qk

Document Version:

Publisher's PDF, also known as Version of record

\section{Please check the document version of this publication:}

- A submitted manuscript is the version of the article upon submission and before peer-review. There can be important differences between the submitted version and the official published version of record.

People interested in the research are advised to contact the author for the final version of the publication, or visit the DOI to the publisher's website.

- The final author version and the galley proof are versions of the publication after peer review.

- The final published version features the final layout of the paper including the volume, issue and page numbers.

Link to publication

\footnotetext{
General rights rights.

- You may freely distribute the URL identifying the publication in the public portal. please follow below link for the End User Agreement:

www.umlib.nl/taverne-license

Take down policy

If you believe that this document breaches copyright please contact us at:

repository@maastrichtuniversity.nl

providing details and we will investigate your claim.
}

Copyright and moral rights for the publications made accessible in the public portal are retained by the authors and/or other copyright owners and it is a condition of accessing publications that users recognise and abide by the legal requirements associated with these

- Users may download and print one copy of any publication from the public portal for the purpose of private study or research.

- You may not further distribute the material or use it for any profit-making activity or commercial gain

If the publication is distributed under the terms of Article $25 \mathrm{fa}$ of the Dutch Copyright Act, indicated by the "Taverne" license above, 


\section{POWER INDICES, CLAIM GAMES AND CORE SELECTION}


(C) Qianqian Kong, Maastricht 2021

Printing: ProefschriftMaken || www.proefschriftmaken.nl

ISBN: 9789464231137

All rights reserved. No part of this work may be reproduced, stored in a retrieval system or transmitted, in any form or by any means, electronic, mechanical, photocopying, recording, or otherwise, without prior permission of the author or the coptyright-owning journals for previous published chapters. 


\section{POWER INDICES, CLAIMS GAMES AND CORE SELECTION}

\section{DISSERTATION}

to obtain the degree of Doctor at the Maastricht University, on the authority of the Rector Magnificus, Prof. dr. Rianne M. Letschert

in accordance with the decision of the Board of Deans, to be defended in public on Monday 8 February 2021 at 13:00 hours

by

Qianqian Kong 
Supervisor:

Prof. dr. H.J.M. Peters

Co-Supervisor:

Prof. dr. H. Sun: Northwestern Polytechnical University, Xi'an, China

Assessment Committee:

Prof. dr. D. Vermeulen (Chair)

Prof. dr. J.-J. Herings

Prof. dr. R. van den Brink: Vrije Universiteit Amsterdam

Prof. dr. B. Klaus: University of Lausanne, Switzerland

Prof. dr. J. Zarzuelo: University of the Basque Country, Spain

Dr. P. Sun: Northwestern Polytechnical University, Xi'an, China 


\section{Acknowledgements}

I am flattered to be the first $\mathrm{PhD}$ candidate of the double degree program between Northwestern Polytechnical University of China and Maastricht University of the Netherlands. With the completion of my doctoral defense my study in Maastricht comes to a perfect end. Before saying Goodbye, I would like to show my great appreciation to everyone who has helped me ever.

With my most sincere respect, I first would like to thank my two supervisors: Hans Peters, my primary supervisor in Maastricht, and Hao Sun, my co-supervisor in Masstricht and primary supervisor in China. The discussions we had at our regular meetings are still fresh in my mind. Thank them both for sharing with me inspirations and scientific tips, and giving me instant feedback. They also affect me in their own ways. Also as my master supervisor Hao took me to begin the truth-seeking journey in Game Theory, either externally or internally. For a better development he always funded me to attend academic conferences to broaden my insights, and encouraged me to apply for the scholarship from China Scholarship Council for further study. Without his suggestion, how could I experience this gorgeous trip in Maastricht. I here show my great appreciation to Hao who makes my academic life colorful! My subsequent $\mathrm{PhD}$ career was spent in Maastricht. Studying abroad is really a challenge, however, I am acclimated to the life in Maastricht easily under Hans' kindness. Even in this epidemic situation, I never felt lonely through the weekly meeting with him. To be honest I have never regarded research as a hobby, but as a stepping stone to work in the future. It is just his passions for scientific research that gradually cultivate my enthusiasm. Without his guidance and influence, I cannot imagine how I could have completed my $\mathrm{PhD}$ thesis in Maastricht. I here show my heartfelt thanks to 
Hans who really broadened my research horizons.

Thanks to Professors Genjiu Xu and Dongshuang Hou from China, who also offered me a lot of help in my $\mathrm{PhD}$ journey. Thanks to them for inspiring me in every seminar. Thanks to them for hosting each conference so that we can have more academic exchanges with outstanding scholars. I would also like to thank the thesis committee members: Dries Vermeulen, René van den Brink, Bettina Klaus, Jose Zarzuelo, Jean-Jacques Herings and Panfei Sun for their time and patience to seriously read this thesis and offer me practical feedback instantly.

Thanks to my fellows and friends who have accompanied me through most of my doctoral career. As the saying goes, one depends upon parents at home and upon friends outside. It is really true. I will always be grateful for their friendships.

Last but not least, I offer my sincere appreciation to my family. Thanks to all of them for their unconditional love, making me stronger and full of anticipation for the future. My soulmate, Kunfeng, deserves my special gratitude. He could be any role whenever I need: my husband in relationships, a good assistant in life, a psychoanalyst in my dealing with setbacks, etc. Thanks a lot for all his giving, my Mr. Right!!

Qianqian Kong

Maastricht

8 February 2021 


\section{Contents}

Acknowledgements v v

1 Introduction 1

2 An issue based power index 5

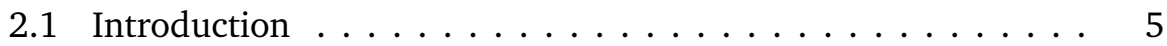

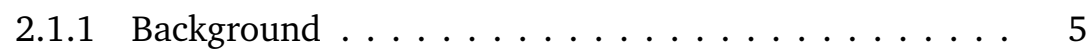

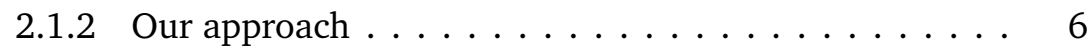

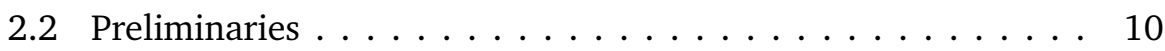

2.3 Issue Dependence and No Advantageous Splitting . . . . . . . 11

2.4 Invariance with respect to Symmetric Players . . . . . . . . 15

2.5 Equal Power Change . . . . . . . . . . . . . . . 17

2.6 Further discussion . . . . . . . . . . . . . . . . . 21

2.6.1 Relation with other power indices . . . . . . . . . 21

2.6 .2 Player preferences . . . . . . . . . . . . . . . 22

3 Power indices for matching problems 25

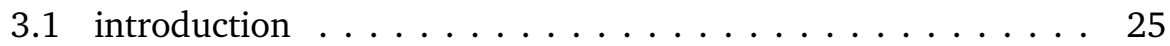

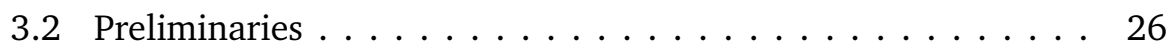

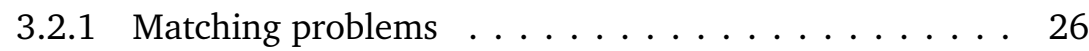

3.2.2 Effectivity functions based on being single . . . . . . 27

3.2.3 Effectivity functions based on blocking coalitions . . . . 28

3.2.4 Power indices and the Transfer Property . . . . . . . 28

3.3 Power in the singles market . . . . . . . . . . . . . . 29 
3.4 Power by blocking . . . . . . . . . . . . . . . 32

Appendix A Remaining proofs of Section $3.3 \ldots \ldots \ldots \ldots$

Appendix B Independence of the axioms in Theorem $3.2 \ldots \ldots$

Appendix C Proofs of Section $3.4 \ldots \ldots \ldots \ldots \ldots$. . . . . 41

Appendix D Independence of the axioms in Theorem 3.3 . . . . 45

4 Sequential claim games 47

4.1 Introduction $\ldots \ldots \ldots \ldots \ldots \ldots \ldots \ldots$. . . . . . . 47

4.2 Model and preliminaries . . . . . . . . . . . . . . . . . . 49

4.2 .1 Actions and claims profiles . . . . . . . . . . 50

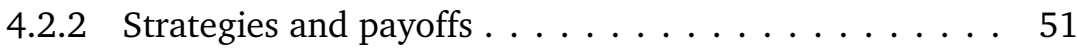

4.2 .3 Myopic strategies . . . . . . . . . . . . . . . . . 51

4.2 .4 Best replies . . . . . . . . . . . . . . . . . 52

4.2.5 Myopic strategies and Nash equilibrium . . . . . . . . 53

4.3 Left-right restricted claims . . . . . . . . . . . . . . . . 55

4.4 Subgame perfect equilibrium . . . . . . . . . . . 60

4.4.1 Subgame perfect equilibrium for $n \in\{2,3,4\} \ldots \ldots 62$

4.4.2 Myopic play with punishment . . . . . . . . . . . 63

4.5 Payoffs . . . . . . . . . . . . . . . . . . . . . . . 64

4.6 Concluding remarks $\ldots \ldots \ldots \ldots \ldots 6$

Appendix A Remaining proofs from Section $4.3 \ldots \ldots$. . . . 67

Appendix B Nash equilibria in $\widetilde{\mathscr{S}}$ for three players . . . . . . . . . . . . . . . . . . . . . . .

Appendix C Proof of Proposition $4.3 \ldots \ldots \ldots \ldots \ldots$

Appendix D Proof of Theorem 4.7 . . . . . . . . . . . . . 79

5 Core selections for transferable utility games 89

5.1 Introduction . . . . . . . . . . . . . . . . . 89

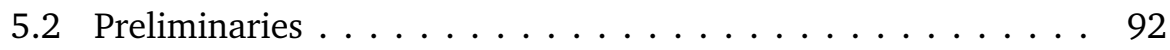

5.3 Associated games to optimize the core . . . . . . . . . . . 94

5.4 Matrix approach to select one outcome from the core . . . . . 101

5.5 Special cases of weight coefficients . . . . . . . . . . . 109

5.6 A numerical illustration . . . . . . . . . . . . . 111 
5.7 Conclusions . . . . . . . . . . . . . . . . 113

$\begin{array}{ll}\text { Bibliography } & 115\end{array}$

$\begin{array}{ll}\text { Summary } & 123\end{array}$

$\begin{array}{ll}\text { Impact of the thesis } & 125\end{array}$

$\begin{array}{ll}\text { About the Author } & 127\end{array}$ 



\section{Chapter 1}

\section{Introduction}

Game theory provides general mathematical techniques for analyzing situations in which two or more individuals make decisions that will influence one another's welfare. The foundations of game theory are laid in the seminal book 'Theory of Games and Economic Behavior' by von Neumann and Mongenstern [68]. Game theoretical approaches are often classified into two branches: cooperative and noncooperative game theory. Cooperative game theory emphasizes group rationality, efficiency and fairness, while noncooperative game theory concentrates on individual rationality and individual optimal decision-making.

This thesis contains three different themes in game theory. Specifically, it has five chapters: after this introductory chapter, Chapters 2 and 3 focus on power indices. Chapter 4 deals with Nash and subgame perfect Nash equilibria in sequential claim games. The last chapter focusses on core selection in cooperative games, recursively optimizing the core to a more stable subset.

In the wake of the seminal contribution of Shapley and Shubik in 1954 [61], a copious literature on so-called 'power indices' in general has been, and continues to be, produced. A power index is a tool to measure and compare the power of players in political and economic situations. Power indices for simple games measure the power of players in such a game independently of the issues at stake or the positions of players regarding these issues. One may well argue that this is how it should be, but one may also argue that this is 
a drawback: neither the position of a political party nor the issues at stake are taken into consideration. The second and third chapters of the thesis deal with this in different contexts.

Chapter 2 considers issue games. An issue game is a combination of a monotonic simple game and an issue profile. An issue profile is a profile of linear orders on the player set, one for each issue within the set of issues: such a linear order is interpreted as the order in which the players will support the issue under consideration. A power index assigns to each player in an issue game a nonnegative number, where these numbers sum up to one. We consider a class of power indices, characterized by weight vectors on the set of issues. A power index in this class assigns to each player the weighted sum of the issues for which that player is pivotal. A player is pivotal for an issue if that player is a pivotal player in the coalition consisting of all players preceding that player in the linear order associated with that issue. The main part of Chapter 2 is devoted to presenting three axiomatic characterizations of this class of power indices.

Based on work in progress, Chapter 3 develops two families of power indices for so-called marriage problems in Gale and Shapley [25] and in Roth and Sotomayor [55]. In this situation a power index is a measure for the possibilities on the marriage market. We approach this question by considering two types of effectivity functions, which reflect the possibilities of the men and women in a marriage problem. In the first approach, we take the situation where every one is single as a point of departure. Then the effectivity function associated with the profile of preferences assigns to every matching those couples in the matching that prefer each other over staying single. In the second approach, again given the profile of preferences, the effectivity function assigns to every matching those couples or individuals that can block the matching. In both approaches, a power index acts on the associated effectivity functions, rather than directly on the preference profile. The main results of Chapter 3 are devoted to proposing a number of axioms that characterize families of power indices.

In an estate division problem, also called bankruptcy problem, a number of players have entitlements on an estate. In general, the sum of these entitlements exceeds the size of the estate, so that the division of the estate becomes 
a problem. In Chapter 4 we take a noncooperative approach by assuming that players sequentially put claims on the estate in a given order. Each part of the estate is then divided proportionally with respect to the number of claims on it. For this so-called sequential claim game, we study strategy profiles that are a Nash equilibrium or, stronger, a subgame perfect equilibrium. We focus on myopic play: this means that a player first claims the hitherto least claimed parts, next the second least claimed parts, and so on and so forth, until the player's entitlement is exhausted. However, we show that myopic strategies do not necessarily form a Nash equilibrium, and that there can be Nash equilibria that are not myopic. We then focus on two questions: how myopic play can be turned into a Nash equilibrium and even a subgame perfect equilibrium. The main results are that myopic play is always a Nash equilibrium provided that claims on the same level are always ordered from left to right, and that left-right myopic play with punishment is always a subgame perfect equilibrium in the general case

The core of a cooperative game with transferable utility is a set that distributes the worth of the grand coalition such that each coalition obtains at least what it could gain on its own. In other words, if the players receive a payoff according to a core allocation, no coalition has any incentive to deviate from the grand coalition. In order to select from the core, we sharpen it to an increasingly justified set by invoking extra criteria. Ideally, we can select a unique point from the core in a game and consider it as the final allocation. This process of narrowing down the core by using reasonable criteria, is also called core optimization, see Chapter 5 . Specifically, for each game we establish a sequence of associated games, recursively. This associated game reflects an optimistic self-assessment of every coalition's worth: a coalition may believe that the appropriation of at least a part of the surplus, generated by cooperation with its complement, is within reach. We show that the cores of the associated games are increasingly stable. The last one of the nonempty cores in this sequence is therefore the final optimized set. 



\section{Chapter 2}

\section{An issue based power index}

\subsection{Introduction}

\subsubsection{Background}

Power indices for simple games measure the power of players in such a game, independently of the issues at stake or the positions of players regarding these issues. For instance, a power index applied to a weighted majority game associated with a political parliament, typically considers how often a political party is needed to form a majority, without taking the issue at stake (for instance, a new law) into account. One may well argue that this is how it should be (for instance, Braham and Holler [9]), but one may also argue that this is a drawback (e.g., Napel and Widgrén [45]).

For a relatively recent overview of power indices for simple games see Bertini et al. [6]. These power indices include the Shapley value [60], also called Shapley-Shubik index [61], the Banzhaf value [4,47,64] and the BanzhafColeman index [12], the Holler index [40], and many more. Most of these power indices, including the ones mentioned, are based on counting in some way or another the number of times a player is pivotal in the simple game.

\footnotetext{
Adapted from: 'Kong Q. and Peters H.: An issue based power index. Forthcoming in International Journal of Game Theory, 2020'.
} 
There are, however, also many approaches which do take the issues at stake, and the preferences of players regarding these issues, into account. Notably, so-called spatial power indices are defined on simple games, enriched by adding positions of the players in policy space. For instance, Owen [49] and later Owen and Shapley [50] add to the simple game a vector of positions of players in two-dimensional Euclidean space, and use this to obtain a variant of the Shapley-Shubik index, called the Owen-Shapley spatial power index, which takes these positions into consideration. See Peters and Zarzuelo [54] for an axiomatic characterization of this index. As will become clear below, the approach in the present chapter is based on a similar idea as the OwenShapley spatial power index. On the topic of spatial power indices, see also Enelow and Hinich [21, 22], Grofman et al. [29], Straffin [65], Felsenthal and Machover [23,24], and Laruelle and Valenciano [41]. More recent contributions are Alonso-Meijide et al. [1], Benatti and Marzetti [5], Martin et al. [42], and Blockmans and Guerry [7].

An alternative and less known definition of a power index is proposed by Hoede and Bakker [33], based on so-called inclination vectors: the positions of players with respect to certain issues may influence each other, and a power index may depend on the strengths of these influences. See also Rusinowska and de Swart [56].

A theory of power measurement within corporate and/or financial networks is proposed by Gambarelli and Owen [26]. See also Karos and Peters [37] and Mercik and Stach [43]. For power measurement on graphs, see e.g. van den Brink [67], or Peters et al. [53]. Karos and Peters [38] discuss power indices when the possibilities of players and coalitions are described by an effectivity function.

Still other approaches model the impact of preferences on power by means of a noncooperative voting game: see, for instance, Schmidtchen and Steunenberg [58].

\subsubsection{Our approach}

Our approach is best illustrated by a simple example. Consider a parliament with four parties, called $1,2,3$, and 4 , which have numbers of seats 49 , 
17, 17, and 17, respectively. To pass any law a strict majority (at least 51 seats) is required. ${ }^{1}$ The Shapley-Shubik and (normalized) Banzhaf values assign power distribution $\frac{1}{6}(3,1,1,1)$, and the Holler index assigns $\frac{1}{9}(3,2,2,2)$. These power distributions are completely independent of what is at stake. Now suppose that during the period that this particular composition of the parliament is in vigor, there are three main issues (say, new laws) under consideration, and suppose that the big party 1 is highly in favor of these issues. This implies that party 1 is practically powerless, since it always depends on some other party (which is less enthusiastic about these issues) in order to pass the corresponding law. Pursuing the example somewhat further, call the issues $a, b$, and $c$, and let the 'order of enthusiasm' for each of the issues be given by $1,2,3,4$ for $a, 1,3,4,2$ for $b$, and $1,4,3,2$ for $c$. If we assume that for each issue a supporting coalition is formed according to the given order, we see that the pivotal party for $a$ would be party 2 , for $b$ party 3 , and for $c$ party 4 . Taking the number of times a party is pivotal as a measure for its power, the resulting power distribution is $\frac{1}{3}(0,1,1,1)$. In a nutshell, this is the (main) issue based power index that we consider in this chapter. ${ }^{2}$

As mentioned above, this approach is closely inspired by the construction of the Owen-Shapley spatial power index [50]. In the spatial game model of Owen and Shapley, each issue is a point $u$ on the unit circle in $\mathbb{R}^{2}$ and each player $i$ has a position $p_{i}$ in $\mathbb{R}^{2}$. Then player $i$ is more enthusiastic about issue $u$ than player $j$ if $p_{i} \cdot u<p_{j} \cdot u$-thus, the inner product is interpreted as linear (dis)utility. A given simple game then determines, for each issue, which player is pivotal: this is the first player who makes the coalition, formed in the order of enthusiasm, winning. As a result, the unit circle is partitioned into subsets with different pivotal players, and the relative size of the subset ( $\operatorname{arc}(\mathrm{s}))$ for which a player is pivotal, is defined to be that player's power according to the Owen-Shapley spatial power index. Here, assuming that all positions are different, the set of issues for which the pivotal player is not unique has measure zero and can be neglected. This is no longer the case

\footnotetext{
${ }^{1}$ The associated simple game is a weighted majority game. In this case, it is also an apex game with player 1 as the apex player.

${ }^{2}$ Of course, it may well be possible that the biggest party 1 has had an important say in setting the agenda. Here, however, we assume that the agenda - set of issues - is given.
} 
if the number of issues is finite, as in the present chapter: this is one of the reasons that we take the orderings of the players per issue, rather than their positions (i.e., their preferences over the issues) as primitives in the model. For more discussion on this choice the reader is referred to the concluding Section 2.6.

Instead of spatial games, in this chapter we consider issue games. An issue game consists of a monotonic simple game for some player set $N$, and for each issue within the set of issues $M$, a linear order over the set of players $N$. This linear order expresses the order of enthusiasm or support of the players for the issue under consideration. Such a collection of linear orders is called an issue profile. In the example above, the simple game is the four-player weighted majority game, and the issue profile is the set of three linear orders over the four players, associated with the issues $a, b$, and $c$. A power index assigns to each player in an issue game a nonnegative number, where these numbers sum up to one. The power indices studied in this chapter are characterized by weight vectors (nonnegative vectors with coordinates summing up to one) for the set of issues, such that the power assigned to each player in an issue game is equal to the weighted sum of the issues for which this player is pivotal. (In the example above, all three issues have equal weight $\frac{1}{3}$.) The main part of this chapter is devoted to presenting several axiomatic characterizations of this class of power indices.

The first result in this chapter is Theorem 2.1, in which this class is characterized by two axioms: Issue Dependence and No Advantageous Splitting. Issue Dependence requires that in a situation where each player is pivotal for at most one issue, the power of a pivotal player depends (only) on the issue at stake. This axiom will imply that we can attach fixed weights to issues. No Advantageous Splitting says that no player, by splitting up into several players, can change the power distribution. For instance, in the above example, if party 2 would split up into two parties of sizes, say, 8 and 9 seats, its total power should stay the same, and also the powers of players 1,3 , and 4 should not change. The axiom is similar in spirit (and equal in name) to an axiom for bankruptcy problem rules in de Frutos [13], where it is considered as a kind of non-manipulability. It is also closely related to the 2-efficiency axiom in Nowak [47], used in a characterization of the Banzhaf index. The proof of 
this result is quite transparent: given an issue game, if a player is pivotal for more than one issue, we split this player up in new players such that each of the new players is pivotal for exactly one issue; and then apply Issue Dependence. In order for this to work, we assume that the set of players may vary, as a finite subset of the universal set of players identified with $\mathbb{N}$. The set of issues is regarded as fixed.

In the second characterization (Theorem 2.2) we consider the axiom of Invariance with respect to Symmetric Players: given any issue game and two players $i$ and $i^{\prime}$ who are symmetric in the associated simple game, if $i$ is pivotal for an issue and we change the linear order of that issue so that $i^{\prime}$ becomes pivotal, then the power assigned to every other player should not change. Additionally, we strengthen Issue Dependence to Strong Issue Dependence: if, in two issue games, the partitions of the set of issues, obtained by grouping together those issues which have the same pivotal player, coincide, then the power assigned to the players should only depend on the issues for which they are pivotal. These two conditions again characterize the same class of power indices. The proof of Theorem 2.2 is in some sense dual to the proof of Theorem 2.1: we start with an issue game in which the simple game is a unanimity game for the grand coalition (in particular, all players are symmetric), and by using Invariance with respect to Symmetric Players, change this to an issue game with any arbitrary partition of the set of issues, by grouping issues together; then, we apply Strong Issue Dependence.

In our third characterization (Theorem 2.5) we use the axiom Equal Power Change, which concerns the simple game rather than the issue profile. This axiom is a variation on the transfer property of Dubey [17] and makes that the power index is uniquely determined by its values on issue games in which the simple games are unanimity games. Adding this axiom, allows for a considerable weakening of Strong Issue Dependence, namely to Symmetric Player Issue Dependence. The two axioms, plus Invariance with respect to Symmetric Players, again characterize the same class of power indices.

The organization of this chapter is as follows. After preliminaries in Section 2.2 the three characterizations are presented in Sections 2.3, 2.4, and 2.5. Section 2.6 concludes with further discussion. 


\subsection{Preliminaries}

We identify the universe of potential players with $\mathbb{N}$. A monotonic simple game is a pair $(N, v)$, where $N \subseteq \mathbb{N}$ is a nonempty finite set of players and $v: 2^{N} \rightarrow$ $\{0,1\}$ satisfies $v(\emptyset)=0, v(N)=1$, and $v(S) \leq v(T)$ whenever $S \subseteq T \subseteq N$. Throughout we only consider simple games that are monotonic, and just refer to these as simple games. A coalition is a subset of $N$. A coalition $S$ is minimal winning if $v(S)=1$ and $v\left(S^{\prime}\right)=0$ for every $S^{\prime} \subsetneq S$. Player $i \in N$ is a null player in the simple game $(N, v)$ if $v(S \cup\{i\})-v(S)=0$ for all $S \subseteq N$. Two players $i$ and $i^{\prime}$ in a simple game $(N, v)$ are symmetric if $v(S \cup\{i\})=v\left(S \cup\left\{i^{\prime}\right\}\right)$ for every $S \subseteq N \backslash\left\{i, i^{\prime}\right\}$. A unanimity game is a simple game $\left(N, u_{T}\right)$ for some $\emptyset \subsetneq T \subseteq N$, defined by $u_{T}(S)=1$ if and only if $T \subseteq S$, for every $S \subseteq N$.

The set of issues is a nonempty finite set $M$; we usually write $M=\{1, \ldots$, $|M|\}$. Throughout, the set of issues is fixed and therefore suppressed from notation. An issue game is a triple $(N, v, Q)$ with $(N, v)$ a simple game and $Q$ a mapping from $M$ to the set of linear orders on $N .^{3}$ The mapping $Q$ is an issue profile; for $j \in M, Q^{j}$ denotes the linear order on $N$ assigned by $Q$ to issue $j$. By $\mathscr{G}$ we denote the set of all issue games. An issue based power index or simply power index is a map $\varphi$ on $\mathscr{G}$ such that for every $G=(N, v, Q) \in \mathscr{G}, \varphi_{i}(G) \geq 0$ for all $i \in N$ and $\sum_{i \in N} \varphi_{i}(G)=1$. Hence, a power index is individually rational and efficient by definition. ${ }^{4}$

In an issue game $G=(N, v, Q)$, player $i \in N$ is pivotal for issue $j \in M$ if $v(S \cup\{i\})-v(S)=1$, where $S=\left\{k \in N: k Q^{j} i\right\}$. In this case we also write $i=p_{G}(j)$, that is, $p_{G}(j)$ denotes the player who is pivotal for issue $j$ in the issue game $G$. We use the notation $M_{G}^{i}=\left\{j \in M: i=p_{G}(j)\right\}$ for the set of issues for which player $i$ is pivotal. Further, $P(G)$ denotes the set of pivotal players in $G$, i.e., $P(G)=\left\{i \in N: M_{G}^{i} \neq \emptyset\right\}$. Clearly, $|P(G)| \leq|M|$. Also, if player $i \in N$ is a null player in the simple game $(N, v)$, then $i \notin P(G)$.

\footnotetext{
${ }^{3} \mathrm{~A}$ linear order $R$ on a set of players $N$ is, as usual, an irreflexive, complete, transitive and asymmetric binary relation on $N$. Instead of $(i, j) \in R$ we write $i R j$.

${ }^{4}$ We include these conditions in the definition since they will be imposed throughout. We also repeat that the set of players may vary, but the set of issues is fixed.
} 
A weight vector is a vector $w \in \mathbb{R}^{M}$ satisfying $w_{j} \geq 0$ for all $j \in M$, and $\sum_{j \in M} w_{j}=1$. The power index $\Phi^{w}$ is defined by

$$
\Phi_{i}^{w}(G)=\sum_{j \in M_{G}^{i}} w_{j}
$$

for every $G=(N, v, Q) \in \mathscr{G}$. Hence, player i's power according to $\Phi^{w}$ in the issue game $G$ is simply the issue-weighted number of times that player $i$ is pivotal. For the special case that all issues always have equal weights, i.e., $w_{j}=\frac{1}{|M|}$ for every $j \in M$, we write $\Phi$.

\subsection{Issue Dependence and No Advantageous Splitting}

Let $\varphi$ be a power index. In this section we consider two axioms for $\varphi$, and show that these characterize the family of power indices $\Phi^{w}$.

The first axiom says that in games where each player is pivotal for at most one issue, the power of each pivotal player depends only on the issue for which that player is pivotal. Observe that in such a situation every pivotal player is pivotal for exactly one issue, and then it is reasonable to assume that the power of each pivotal player depends exclusively on the issue for which that player is pivotal.

Issue Dependence (ID) For all $G=(N, v, Q) \in \mathscr{G}$ and $\widehat{G}=(\widehat{N}, \widehat{v}, \widehat{Q}) \in \mathscr{G}$ such that $|P(G)|=|P(\widehat{G})|=|M|$, and all $j \in M, \varphi_{p_{G}(j)}(G)=\varphi_{p_{\widehat{G}}(j)}(\widehat{G})$.

The second axiom says that players should not be able to increase (or decrease) their power by splitting up into more than one separate players. Think of a (pivotal) political party which could split up into smaller, still pivotal, parties: this should not influence the distribution of power within a parliament. More precisely, suppose that the game $\widehat{G}$ arises from the game $G$ by (a) replacing player $i$, pivotal in $G$, by player set $I$, disjoint with the player set $N$ in $G$, (b) changing the simple game only in the sense that in $\widehat{G}$ every coalition of players containing at least one player from $I$ has the same worth as the original coalition with player $i$, and (c) replacing in the linear order associated with any issue $j$, player $i$ by the player set $I$ in any arbitrary order, 
without changing the rest of the order for issue $j$. The axiom then requires that the original power of $i$ in $G$ is equal to the total power of the players of $I$ in the new situation $\widehat{G}$, whereas all the other players just keep their original power. Formally:

No Advantageous Splitting (NAS) For every $G=(N, v, Q) \in \mathscr{G}$, every $i \in$ $P(G)$, every player set $I$ with $I \cap N=\emptyset$, and every $\widehat{G}=(I \cup N \backslash\{i\}, \widehat{v}, \widehat{Q})$ such that

- $\widehat{v}(S)=v(S)$ for all $S \subseteq N \backslash\{i\}$ and $\widehat{v}(S)=v(\{i\} \cup(S \backslash I))$ for every $S \subseteq N \cup I$ with $S \cap I \neq \emptyset$, and

- for every $j \in M, \widehat{Q}^{j}$ satisfies $k \widehat{Q}^{j} k^{\prime}$ if and only if $k Q^{j} k^{\prime}$, for all $k, k^{\prime} \in$ $N \backslash\{i\}$; and $k \widehat{Q}^{j} i^{\prime}$ if and only if $k Q^{j} i$, for all $k \in N \backslash\{i\}$ and $i^{\prime} \in I$,

we have

$$
\varphi_{i}(G)=\sum_{i^{\prime} \in I} \varphi_{i^{\prime}}(\widehat{G}) \text { and } \varphi_{\ell}(G)=\varphi_{\ell}(\widehat{G}) \text { for all } \ell \in N \backslash\{i\}
$$

As already mentioned in the Introduction, an axiom with the same name and spirit occurs in de Frutos [13] in the context of bankruptcy problems. There, combined with an analogous condition of 'no advantageous merging', it is interpreted as a non-manipulability condition. It is also closely related to the '2-efficiency' condition in Nowak [47], which is used in a characterization of the normalized Banzhaf value without additivity.

We now show that these two axioms characterize the family of power indices $\Phi^{w}$.

Theorem 2.1. Let $\varphi$ be a power index. Then $\varphi$ satisfies ID and NAS, if and only if there is a weight vector $w$ such that $\varphi=\Phi^{w}$.

Proof. We leave verification of the if-part of the theorem to the reader. For the only-if part, assume that $\varphi$ satisfies ID and NAS.

First, we fix an issue game $\widehat{G}=(\widehat{N}, \widehat{v}, \widehat{Q})$ such that $P(\widehat{G})=\widehat{N}$ and $|P(\widehat{G})|=$ $|M|$. We define the vector $w \in \mathbb{R}^{M}$ by $w_{j}=\varphi_{p_{\widehat{G}}(j)}(\widehat{G})$ for every $j \in M$. Then $w$ is a weight vector since, in particular, $|P(\widehat{G})|=|\widehat{N}|=|M|$. 
Now let $G=(N, v, Q) \in \mathscr{G}$ be an arbitrary issue game. Without loss of generality let $P(G)=\{1, \ldots, s\}$, where $s \leq|M|$. Note that $M_{G}^{1}, \ldots, M_{G}^{s}$ is a partition of $M$. Since $w$ is a weight vector, it is sufficient to prove that $\varphi_{\ell}(G)=\sum_{j \in M_{G}^{\ell}} w_{j}$ for every $\ell \in P(G)$.

We start with constructing an issue game $G_{1}$ in which player 1 is replaced by a collection of new players $I_{1}$, as many as the number of issues for which player 1 is pivotal, i.e., $\left|I_{1}\right|=\left|M_{G}^{1}\right|$. Without loss of generality, write $M_{G}^{1}=$ $\{1, \ldots, \ell\}$ and $I_{1}=\left\{1_{1}, \ldots, 1_{\ell}\right\}$, such that $I_{1} \cap N=\emptyset$. Define the simple game $\left(I_{1} \cup N \backslash\{1\}, v_{1}\right)$ by $v_{1}(S)=v(S)$ whenever $S \subseteq N \backslash\{1\}$, and $v_{1}(S)=$ $v\left(\{1\} \cup S \backslash I_{1}\right)$ otherwise. Define the issue profile $Q_{1}$ as follows. For $j \in M \backslash M_{G}^{1}$ let $Q_{1}^{j}$ be such that $k Q_{1}^{j} k^{\prime}$ if and only if $k Q^{j} k^{\prime}$, for all $k, k^{\prime} \in N \backslash\{1\}$; and $k Q_{1}^{j} 1^{\prime}$ if and only if $k Q^{j} 1$, for all $k \in N \backslash\{1\}$ and $1^{\prime} \in I_{1}$. For $j=1, \ldots, \ell$ let $Q_{1}^{j}$ be such that $k Q_{1}^{j} k^{\prime}$ if and only if $k Q^{j} k^{\prime}$, for all $k, k^{\prime} \in N \backslash\{1\} ; k Q_{1}^{j} 1^{\prime}$ if and only if $k Q^{j} 1$, for all $k \in N \backslash\{1\}$ and $1^{\prime} \in I_{1}$; and $1_{j} Q_{1}^{j} 1_{j^{\prime}}$ for all $j^{\prime} \in\{1, \ldots, \ell\} \backslash\{j\}$.

In words, in the new profile $Q_{1}$, everywhere player 1 is replaced by the player set $I_{1}$ such that for the issues where player 1 was pivotal, each time a different player from $I_{1}$ is pivotal. In particular, in the new issue game $G_{1}=\left(I_{1} \cup N \backslash\{1\}, v_{1}, Q_{1}\right)$ every player in $I_{1}$ is pivotal exactly once. By NAS we have

$$
\varphi_{1}(G)=\sum_{i \in I_{1}} \varphi_{i}\left(G_{1}\right) \text {, and } \varphi_{i}(G)=\varphi_{i}\left(G_{1}\right) \text { for all } i \in N \backslash\{1\}
$$

Next, we apply a similar construction to $G_{1}$ by replacing player 2 by player set $I_{2}$ with $\left|I_{2}\right|=\left|M_{G}^{2}\right|$ and with $I_{2} \cap\left(N \cup I_{1}\right)=\emptyset$, resulting in a new issue game $G_{2}$ for which by NAS we have

$$
\varphi_{2}(G)=\varphi_{2}\left(G_{1}\right)=\sum_{i \in I_{2}} \varphi_{i}\left(G_{2}\right) \text { and } \varphi_{i}\left(G_{1}\right)=\varphi_{i}\left(G_{2}\right) \text { for all } i \in I_{1} \cup N \backslash\{1,2\} \text {. }
$$

Repeating this construction for players $3, \ldots, s$, we end up with an issue game $G_{s}$ with player set $I_{1} \cup \ldots \cup I_{s} \cup(N \backslash P(G))$ for which $\left|P\left(G_{s}\right)\right|=\left|I_{1} \cup \ldots \cup I_{s}\right|=|M|$, 
and where by repeated application of NAS we have

$$
\varphi_{\ell}(G)=\varphi_{\ell}\left(G_{\ell-1}\right)=\sum_{i \in I_{\ell}} \varphi_{i}\left(G_{\ell}\right)=\sum_{i \in I_{\ell}} \varphi_{i}\left(G_{s}\right) \text { for every } \ell=1, \ldots, s .
$$

By ID, comparing $G_{s}$ and $\widehat{G}$, we obtain

$$
\varphi_{i}\left(G_{s}\right)=w_{j} \text { for } j \in M \text { such that } p_{G_{s}}(j)=i \text {, for every } i \in I_{1} \cup \ldots \cup I_{s} \text {. }
$$

By (2.1) and (2.2) we have

$$
\varphi_{\ell}(G)=\sum_{i \in I_{\ell}} \varphi_{i}\left(G_{s}\right)=\sum_{j \in M_{G}^{\ell}} w_{j} \text { for every } \ell=1, \ldots, s,
$$

which is what we had to prove.

Observe that in the above proof we need the assumption of an infinite universe of potential players: if not, then the proof would not work if all potential players are already involved in the issue game, since then we cannot add new players.

The axioms in Theorem 2.1 are independent, as the following examples show. Verification of the claims in these examples is left to the reader.

\section{Example 2.1.}

(i) For every $G=(N, v, Q) \in \mathscr{G}$ and every $i \in N$ define

$$
\varphi_{i}^{1}(G)= \begin{cases}0 & \text { if } i \notin P(G) \\ \frac{1}{|P(G)|} & \text { if } i \in P(G) .\end{cases}
$$

Then $\varphi^{1}$ is a power index satisfying ID, but not NAS.

(ii) We define $\varphi^{2}$ as follows. Let $M=\{1,2\}, w=(1,0)$, and $\bar{w}=(0,1)$. We define $\varphi^{2}$ by

$$
\varphi^{2}(N, v, Q)= \begin{cases}\Phi^{w}(N, v, Q) & \text { if }(N, v) \text { has no null players } \\ \Phi^{\bar{w}}(N, v, Q) & \text { otherwise. }\end{cases}
$$

Then $\varphi^{2}$ is a power index satisfying NAS, but not ID. 
In case there is no reason to distinguish between issues in terms of importance, the following condition is plausible.

Equal Treatment of Issues (ETI) For all $G=(N, v, Q) \in \mathscr{G}$ such that $|P(G)|=$ $|M|, \varphi_{i}(G)=\varphi_{k}(G)$ for all $i, k \in P(G)$.

It is easy to verify that ETI implies ID. The following result is a straightforward consequence of Theorem 2.1.

Corollary 2.1. Let $\varphi$ be a power index. Then $\varphi$ satisfies ETI and NAS, if and only if $\varphi=\Phi$.

Clearly, the conditions in Corollary 2.1 are again independent. In particular, for independence of ETI, any $\Phi^{w} \neq \Phi$ can be considered.

\subsection{Invariance with respect to Symmetric Players}

In this section we consider the following condition for a power index $\varphi$.

Invariance with respect to Symmetric Players (ISP) For all $G=(N, v, Q)$, $\widehat{G}=(N, v, \widehat{Q}) \in \mathscr{G}$ such that there are $j \in M$ and symmetric players $i, i^{\prime}$ in $(N, v)$ with $\widehat{Q}^{\ell}=Q^{\ell}$ for all $\ell \in M \backslash\{j\}, p_{G}(j)=i$ and $p_{\widehat{G}}(j)=i^{\prime}$, we have $\varphi_{k}(G)=\varphi_{k}(\widehat{G})$ for every $k \neq i, i^{\prime}$.

This condition says that if we replace, for one issue, the pivotal player by another player, where these two players are symmetric in the simple game, then this does not change the power of any of the players not involved in this switch. Of course, this also implies that, with notations as in the definition,

$$
\varphi_{i}(G)+\varphi_{i^{\prime}}(G)=\varphi_{i}(\widehat{G})+\varphi_{i^{\prime}}(\widehat{G})
$$

due to efficiency of a power index. We will show that NAS in Theorem 2.1 can be replaced by ISP if, additionally, we strengthen Issue Dependence (ID) to the following property.

Strong Issue Dependence (SID) For all $G=(N, v, Q), \widehat{G}=(\widehat{N}, \widehat{v}, \widehat{Q}) \in \mathscr{G}$, if the partitions $\left(M_{G}^{i}\right)_{i \in P(G)}$ and $\left(M_{\widehat{G}}^{i}\right)_{i \in P(\widehat{G})}$ are equal, then $\varphi_{p_{G}(j)}(G)=\varphi_{p_{\widehat{G}}(j)}(\widehat{G})$ for every $j \in M$. 
Any issue game induces a partition of its set of issues: the issues in each element of this partition share the same pivotal player. SID says that if in two issue games these partitions coincide, then the power of the player who is pivotal for the issues in an element of the partition in one issue game, should be equal to the power of the player pivotal for the issues in the same partition element, in the other issue game. Clearly, for the case where the two partitions in this definition are equal to the partition of the set of issues into singletons, this condition reduces to ID. Hence, SID is indeed stronger than ID. We need this strengthening since, without the NAS property, ID has no implications for issue games where the number of pivotal players is lower than the number of issues.

The announced theorem is as follows.

Theorem 2.2. Let $\varphi$ be a power index. Then $\varphi$ satisfies SID and ISP, if and only if there is a weight vector $w$ such that $\varphi=\Phi^{w}$.

Proof. It is straightforward to verify that each power index $\Phi^{w}$ satisfies SID and ISP.

For the only-if direction, suppose $\varphi$ satisfies these two conditions. As in the proof of Theorem 2.1, we fix an issue game $\widehat{G}=\left(\widehat{N}, u_{\widehat{N}}, \widehat{Q}\right)$ such that $P(\widehat{G})=\widehat{N}$ and $|P(\widehat{G})|=|M|$. (We now take as simple game the unanimity game $\left(\widehat{N}, u_{\widehat{N}}\right)$, since in this simple game all players are symmetric.) We define the vector $w \in \mathbb{R}^{M}$ by $w_{j}=\varphi_{p_{\widehat{G}}(j)}(\widehat{G})$ for every $j \in M$. Then $w$ is a weight vector since, in particular, $|P(\widehat{G})|=|\widehat{N}|=|M|$.

Now let $G=(N, v, Q) \in \mathscr{G}$ be an arbitrary issue game. Without loss of generality let $P(G)=\{1, \ldots, s\}$, where $s \leq|M|$. Then $M_{G}^{1}, \ldots, M_{G}^{s}$ is a partition of $M$. Since $w$ is a weight vector, it is sufficient to prove that $\varphi_{\ell}(G)=$ $\sum_{j \in M_{G}^{\ell}} w_{j}$ for every $\ell \in P(G)$.

Consider, first, the set $M_{G}^{1}$, take some $j_{1} \in M_{G}^{1}$, and let $k_{1} \in P(\widehat{G})$ with $k_{1}=$ $p_{\widehat{G}}\left(j_{1}\right)$. Define $\widehat{G}_{1}=\left(\widehat{N}, u_{\widehat{N}}, \widehat{Q}_{1}\right) \in \mathscr{G}$ by letting $\widehat{Q}_{1}^{j}=\widehat{Q}^{j}$ for all $j \in M \backslash M_{G}^{1}$, and with $M_{\widehat{G}_{1}}^{k_{1}}=M_{G}^{1}$. (Hence, in $\widehat{G}_{1}$ player $k_{1}$ is pivotal for all issues in $M_{G}^{1}$, i.e., all issues for which player 1 is pivotal in $G$.) By repeated application of ISP, noting that in $\left(\widehat{N}, u_{\widehat{N}}\right)$ all players are symmetric, it follows that $\varphi_{i}\left(\widehat{G}_{1}\right)=\varphi_{i}(\widehat{G})$ for all $i \in P\left(\widehat{G}_{1}\right) \backslash\left\{k_{1}\right\}$. Hence, $\varphi_{k_{1}}\left(\widehat{G}_{1}\right)=\sum_{j \in M_{G}^{1}} w_{j}$, and for every $i \in P\left(\widehat{G}_{1}\right) \backslash\left\{k_{1}\right\}$ we have $\varphi_{i}\left(\widehat{G}_{1}\right)=w_{j}$ for $i=p_{\widehat{G}_{1}}(j)$. 
Next, consider the set $M_{G}^{2}$, take some $j_{2} \in M_{G}^{2}$, and let $k_{2} \in P(\widehat{G})$ such that $k_{2}=p_{\widehat{G}}\left(j_{2}\right)$. Define $\widehat{G}_{2}=\left(\widehat{N}, u_{\widehat{N}}, \widehat{Q}_{2}\right) \in \mathscr{G}$ by letting $\widehat{Q}_{2}^{j}=\widehat{Q}_{1}^{j}$ for all $j \in M \backslash M_{G}^{2}$, and with $M_{\widehat{G}_{2}}^{k_{2}}=M_{G}^{2}$. (Hence, in $\widehat{G}_{2}$ player $k_{2}$ is pivotal for all issues in $M_{G}^{2}$.) Again, by repeated application of ISP it follows that $\varphi_{i}\left(\widehat{G}_{2}\right)=$ $\varphi_{i}\left(\widehat{G}_{1}\right)$ for all $i \in P\left(\widehat{G}_{2}\right) \backslash\left\{k_{2}\right\}$. Hence, $\varphi_{k_{2}}\left(\widehat{G}_{2}\right)=\sum_{j \in M_{G}^{2}} w_{j}, \varphi_{k_{1}}\left(\widehat{G}_{2}\right)=$ $\varphi_{k_{1}}\left(\widehat{G}_{1}\right)=\sum_{j \in M_{G}^{1}} w_{j}$, and for every $i \in P\left(\widehat{G}_{2}\right) \backslash\left\{k_{1}, k_{2}\right\}$ we have $\varphi_{i}\left(\widehat{G}_{2}\right)=w_{j}$ for $i=p_{\widehat{G}_{2}}(j)$.

By repeating this construction we obtain, after $s$ steps in total, an issue game $\widehat{G}_{s}=\left(\widehat{N}, u_{\widehat{N}}, \widehat{Q}_{s}\right)$ with $P\left(\widehat{G}_{s}\right)=\left\{k_{1}, \ldots, k_{s}\right\}$ and such that $M_{\widehat{G}_{s}}^{k_{\ell}}=M_{G}^{\ell}$, and $\varphi_{k_{\ell}}\left(\widehat{G}_{s}\right)=\sum_{j \in M_{G}^{\ell}} w_{j}$, for every $\ell=1, \ldots, s$. By SID applied to $\widehat{G}_{s}$ and $G$, we obtain $\varphi_{\ell}(G)=\varphi_{k_{\ell}}\left(\widehat{G}_{s}\right)=\sum_{j \in M_{G}^{\ell}} w_{j}$ for every $\ell=1, \ldots, s$. This completes the proof.

Note that the proof of Theorem 2.2 parallels that of Theorem 2.1, but in a converse way. In the proof of Theorem 2.1 we use the NAS property to split a player who is pivotal for a subset of issues, into a number of new players such that each new player is pivotal for exactly one issue. In the proof of Theorem 2.2 we use the ISP property to merge several different players, pivotal for different issues, into one player pivotal for the set of those issues.

We can also use the same power indices as in Example 2.1 to show independence of the axioms.

\section{Example 2.2.}

(i) Power index $\varphi^{1}$ in Example 2.1(i) satisfies SID but not ISP.

(ii) Power index $\varphi^{2}$ in Example 2.1(ii) satisfies ISP but not SID.

\subsection{Equal Power Change}

For our last characterization, we consider a condition which is closely related to the familiar condition of additivity for solutions of cooperative games in Shapley [60], and more specifically to the transfer property for solutions of simple games $[17,18]$. Compared to the other axioms, this condition (which is also used in Peters and Zarzuelo [54]) is concerned with the simple game in an issue game, rather than the issue profile. 
Equal Power Change (EPC) For all issue games $(N, v, Q),\left(N, v^{\prime}, Q\right),(N, w$, $Q)$, and $\left(N, w^{\prime}, Q\right)$, if $v(S)-v^{\prime}(S)=w(S)-w^{\prime}(S) \geq 0$ for all $S \subseteq N$, then

$$
\varphi_{i}(N, v, Q)-\varphi_{i}\left(N, v^{\prime}, Q\right)=\varphi_{i}(N, w, Q)-\varphi_{i}\left(N, w^{\prime}, Q\right) \text { for every } i \in N .
$$

In words, given a fixed issue profile, if $v$ arises from $v^{\prime}$ and $w$ arises from $w^{\prime}$ by adding the same winning coalitions, then for every player the change in power in both transitions should be equal.

We first show that every power index $\Phi^{w}$ has this property.

Lemma 2.3. For every weight vector $w, \Phi^{w}$ satisfies EPC.

Proof. Let $N, Q, v, v^{\prime}, w, w^{\prime}$ as in the definition of EPC. Let $j \in M, i \in N$, and let $Q^{j}=\left\{i_{1}, \ldots, i_{s}, \ldots, i_{t}, \ldots, i_{n}\right\}$, where $i_{s}$ is a pivotal player for issue $j$ according to $(N, v)$, and $i_{t}$ is a pivotal player for issue $j$ according to $\left(N, v^{\prime}\right)$. Since $v \geq v^{\prime}$, we have $s \leq t$. We consider the following cases.

1. If $i=i_{s}$ and $s<t$, we have $v\left(i_{1}, \ldots, i_{s}\right)=1,{ }^{5} v\left(i_{1}, \ldots, i_{s-1}\right)=v^{\prime}\left(i_{1}, \ldots, i_{s}\right)=$ $v^{\prime}\left(i_{1}, \ldots, i_{s-1}\right)=0$. Since $v-v^{\prime}=w-w^{\prime} \geq 0$, we have $w\left(i_{1}, \ldots, i_{s}\right)=1$, $w\left(i_{1}, \ldots, i_{s-1}\right)=w^{\prime}\left(i_{1}, \ldots, i_{s}\right)=w^{\prime}\left(i_{1}, \ldots, i_{s-1}\right)=0$. This means player $i$ is pivotal for $j$ according to $(N, w)$ but not pivotal for $j$ according to $\left(N, w^{\prime}\right)$.

2. If $i=i_{t}$ and $s<t$, we have $v\left(i_{1}, \ldots, i_{t}\right)=v\left(i_{1}, \ldots, i_{t-1}\right)=v^{\prime}\left(i_{1}, \ldots, i_{t}\right)=1$ and $v^{\prime}\left(i_{1}, \ldots, i_{t-1}\right)=0$. Since $v-v^{\prime}=w-w^{\prime} \geq 0$, it holds that $w\left(i_{1}, \ldots, i_{t}\right)=$ $w\left(i_{1}, \ldots, i_{t-1}\right)=w^{\prime}\left(i_{1}, \ldots, i_{t}\right)=1$ and $w^{\prime}\left(i_{1}, \ldots, i_{t-1}\right)=0$. This means player $i$ is pivotal for $j$ according to $\left(N, w^{\prime}\right)$ but not pivotal for $j$ according to $(N, w)$.

3. If $i \neq i_{s}$ and $i \neq i_{t}$ and $s<t$, we have the following three cases. We write $i=i_{k}$.

(i) If $k<s$, then, as in Case $1, w\left(i_{1}, \ldots, i_{s}\right)=1$ and $w\left(i_{1}, \ldots, i_{s-1}\right)=0$. Hence, $w\left(i_{1}, \ldots, i_{k}\right)=w^{\prime}\left(i_{1}, \ldots, i_{k}\right)=0$ since $w\left(i_{1}, \ldots, i_{s-1}\right) \geq w\left(i_{1}, \ldots\right.$, $\left.i_{k}\right)$ and $w \geq w^{\prime}$. Thus, $i$ is not pivotal for $j$ according to both $(N, w)$ and $\left(N, w^{\prime}\right)$.

(ii) If $s<k<t$, then $v\left(i_{1}, \ldots, i_{k}\right)=v\left(i_{1}, \ldots, i_{k-1}\right)=1$ and $v^{\prime}\left(i_{1}, \ldots, i_{k}\right)=$ $v^{\prime}\left(i_{1}, \ldots, i_{k-1}\right)=0$. Hence, $w\left(i_{1}, \ldots, i_{k}\right)=w\left(i_{1}, \ldots, i_{k-1}\right)=1$ and

\footnotetext{
${ }^{5}$ We write $v\left(i_{1}, \ldots, i_{s}\right)$ instead of $v\left(\left\{i_{1}, \ldots, i_{s}\right\}\right)$, etc.
} 
$w^{\prime}\left(i_{1}, \ldots, i_{k}\right)=w^{\prime}\left(i_{1}, \ldots, i_{k-1}\right)=0$. Thus, $i$ is not pivotal for $j$ according to both $(N, w)$ and $\left(N, w^{\prime}\right)$.

(iii) If $k>t$, then $v\left(i_{1}, \ldots, i_{k}\right)=v\left(i_{1}, \ldots, i_{k-1}\right)=1$ and $v^{\prime}\left(i_{1}, \ldots, i_{k}\right)=$ $v^{\prime}\left(i_{1}, \ldots, i_{k-1}\right)=1$. Therefore, $w\left(i_{1}, \ldots, i_{k}\right)=w^{\prime}\left(i_{1}, \ldots, i_{k}\right) \geq w\left(i_{1}, \ldots\right.$, $\left.i_{k-1}\right)=w^{\prime}\left(i_{1}, \ldots, i_{k-1}\right)$ since $v-v^{\prime}=w-w^{\prime} \geq 0$. Thus, either player $i$ is pivotal for $j$ according to both $(N, w)$ and $\left(N, w^{\prime}\right)$, or player $i$ is not pivotal for $j$ according to both $(N, w)$ and $\left(N, w^{\prime}\right)$.

4. If $i=i_{s}=i_{t}$, then $v\left(i_{1}, \ldots, i_{s}\right)=v^{\prime}\left(i_{1}, \ldots, i_{s}\right)=1$ and $v\left(i_{1}, \ldots, i_{s-1}\right)=$ $v^{\prime}\left(i_{1}, \ldots, i_{s-1}\right)=0$. Since $v-v^{\prime}=w-w^{\prime} \geq 0$, we have $w\left(i_{1}, \ldots, i_{s}\right)=$ $w^{\prime}\left(i_{1}, \ldots, i_{s}\right) \geq w\left(i_{1}, \ldots, i_{s-1}\right)=w^{\prime}\left(i_{1}, \ldots, i_{s-1}\right)$. Thus, either player $i$ is pivotal for $j$ both in $(N, w)$ and $\left(N, w^{\prime}\right)$, or player $i$ is not pivotal for $j$ according to both $(N, w)$ and $\left(N, w^{\prime}\right)$.

5. If $i \neq i_{s}=i_{t}$, we have the following two cases.

(i) $k<s$. This case is similar to Case 3(i).

(ii) $k>t$. This case is similar to Case 3(iii).

By Cases $1-5$, it follows that $\Phi^{w}(N, v, Q)-\Phi^{w}\left(N, v^{\prime}, Q\right)=\Phi^{w}(N, w, Q)-\Phi^{w}\left(N, w^{\prime}, Q\right)$ for every weight vector $w$.

The next lemma implies that, if $\varphi$ satisfies EPC, then it is completely determined by its values on issue games based on unanimity games. The lemma follows from Lemma 2.3 in Einy [19], see also Einy and Haimanko [20].

Lemma 2.4. Let $\varphi$ be a power index satisfying EPC and let $(N, v, Q)$ be an issue game. Let $T_{1}, \ldots, T_{k}$ be the minimal winning coalitions in $(N, v)$. Then

$$
\varphi(N, v, Q)=\sum_{\emptyset \neq I \subseteq\{1, \ldots, k\}}(-1)^{|I|+1} \varphi\left(N, u_{\cup_{m \in I} T_{m}}, Q\right) .
$$

Adding EPC to the conditions in Theorem 2.2 will allow us to weaken the SID property, by restricting it to players who are symmetric in the associated simple games, as follows.

Symmetric Player Issue Dependence (SPID) For all $G=(N, v, Q), \widehat{G}=$ $(\widehat{N}, \widehat{v}, \widehat{Q}) \in \mathscr{G}$, such that 
- all players in $P(G)$ are symmetric in $(N, v)$ and all players in $P(\widehat{G})$ are symmetric in $(\widehat{N}, \widehat{v})$, and

- the partitions $\left(M_{G}^{i}\right)_{i \in P(G)}$ and $\left(M_{\widehat{G}}^{i}\right)_{i \in P(\widehat{G})}$ are equal,

we have $\varphi_{p_{G}(j)}(G)=\varphi_{p_{\widehat{G}}(j)}(\widehat{G})$ for every $j \in M$.

Theorem 2.5. Let $\varphi$ be a power index. Then $\varphi$ satisfies SPID, ISP, and EPC, if and only if there is a weight vector $w$ such that $\varphi=\Phi^{w}$.

Proof. The if-direction follows from Theorem 2.2 and Lemma 2.3. For the proof of the only-if direction we copy the proof of Theorem 2.2, with now $G$ in that proof of the form $G=\left(N, u_{T}, Q\right)$. The proof is then complete by applying Lemma 2.4 .

The independence of the conditions in Theorem 2.5 is demonstrated by the power indices in the following example.

\section{Example 2.3.}

(i) For each issue game $G=(N, v, Q)$ where $(N, v)$ is a unanimity game, define

$$
\varphi_{i}^{3}(G)= \begin{cases}0 & \text { if } i \notin P(G) \\ \frac{1}{|P(G)|} & \text { if } i \in P(G)\end{cases}
$$

for every $i \in N$. Hence, $\varphi^{3}$ coincides with $\varphi^{1}$ for unanimity games. For an arbitrary issue game $G$, define $\varphi^{3}(G)$ by (2.4). Then $\varphi^{3}$ is a power index satisfying SPID and EPC, but not ISP.

(ii) We define $\varphi^{4}$ as follows. Let $M=\{1,2\}, w=(1,0)$, and $\bar{w}=(0,1)$. Then, for every $G=(N, v, Q)$ with $(N, v)$ a unanimity game,

$$
\varphi^{4}(N, v, Q)= \begin{cases}\Phi^{w}(N, v, Q) & \text { if }(N, v) \text { has no null players } \\ \Phi^{\bar{w}}(N, v, Q) & \text { otherwise. }\end{cases}
$$

Hence, $\varphi^{4}$ coincides with $\varphi^{2}$ for unanimity games. For an arbitrary issue game $G$, define $\varphi^{4}(G)$ by (2.4). Then $\varphi^{4}$ is a power index satisfying ISP and EPC, but not SPID. 
(iii) Define

$$
\varphi^{5}(N, v, Q)=\frac{1}{2^{k}-1} \sum_{\emptyset \neq I \subseteq\{1, \ldots, k\}} \Phi\left(N, u_{\cup_{m \in I} T_{m}}, Q\right),
$$

for every issue game $(N, v, Q)$, where $T_{1}, \ldots, T_{k}$ are the minimal winning coalitions in $(N, v)$ (cf. Lemma 2.4). Then $\varphi^{5}$ is a power index satisfying ISP and SPID, but not EPC. (This example is analogous to an example in Peters and Zarzuelo [54]).

\subsection{Further discussion}

Naturally, there are further questions which could be explored.

The power indices in this chapter are defined and characterized for a fixed set of issues; indeed, all axioms that we consider are formulated for this fixed set of issues. In fact, the results extend straightforwardly if different sets of issues are allowed, but in that case they imply no relation between the associated weight vectors. However, it would not be unnatural to assume that if an issue $j$ is more important than an issue $j^{\prime}$ within a set of issues $M$, then that is still the case within some other set of issues $M^{\prime}$ containing both $j$ and $j^{\prime}$.

Also, simple games in an issue game are exclusively used to determine the pivotalness of a player for an issue; but one might, for instance, also want to take into account the number of times that a player, even if not pivotal, is in a winning coalition resulting from the issue profile - think of player 1 in the example in Section 2.1.2.

We conclude with establishing some relations with other power indices, and with a discussion on the relation with preferences of players over alternatives.

\subsubsection{Relation with other power indices}

By fixing specific issue profiles we obtain several well-known power indices. We consider three examples. Fix a player set $N$ with $|N|=n$. 
(i) Let $M=\{1, \ldots, n !\}$ and let $Q$ be an issue profile such that $Q^{j} \neq Q^{k}$ for all $j, k \in M$ with $j \neq k$. Hence, every permutation of the player set $N$ is assigned to some issue by $Q$. Then $\Phi(N, v, Q)$ is the Shapley value for every simple game $(N, v)$.

(ii) For a simple game $(N, v)$, and for every $i \in N$, denote $M_{i}^{v}=\{S \subseteq$ $N: v(S \cup\{i\})-v(S)=1\}$. Let $M$ be a set of issues with $|M|=\sum_{i \in N}\left|M_{i}^{v}\right|$ such that the elements of $M$ correspond to the pairs $(i, S)$ with $S \in M_{i}^{v}$. Let $Q$ be an issue profile such that for each $j=(i, S) \in M$ we have $k Q^{j} i Q^{j} k^{\prime}$ for all $k \in S$ and $k^{\prime} \in N \backslash(S \cup\{i\})$. Then $\Phi(N, v, Q)$ is the normalized Banzhaf value of $(N, v)$.

(iii) Let $(N, v)$ be a simple game, and let $\mathscr{W}$ be the set of minimal winning coalitions of $(N, v)$. For every $i \in N$ denote $W_{i}^{v}=\{S \in \mathscr{W}: i \in S\}$. Let $M$ be a set of issues with $|M|=\sum_{i \in N}\left|W_{i}^{v}\right|$ such that the elements of $M$ correspond to the pairs $(i, S)$ with $S \in W_{i}^{v}$. Let $Q$ be an issue profile such that for each $j=(i, S) \in M$ we have $k Q^{j} i Q^{j} k^{\prime}$ for all $k \in S$ and $k^{\prime} \in N \backslash(S \cup\{i\})$. Then $\Phi(N, v, Q)$ is the Holler index of $(N, v)$.

\subsubsection{Player preferences}

As noted in the Introduction, an alternative approach, closer to the approach in Owen and Shapley [50], would be to take the profile of preferences of players over issues as a primitive in the model. The following example illustrates how this could work.

Example 2.4. Let $N=\{1, \ldots, 4\}$. Suppose that there are three alternatives $a, b$, and $c$, and that the players have preferences on these alternatives as in the following table:

\begin{tabular}{llll}
\hline 1 & 2 & 3 & 4 \\
\hline$a$ & $a$ & $b$ & $b$ \\
$b$ & $b$ & $a$ & $c$ \\
$c$ & $c$ & $c$ & $a$ \\
\hline
\end{tabular}

Hence, player 1 prefers $a$ over $b$ and $b$ over $c$, etc. We may translate these preferences to an issue profile as follows. For alternative $a$, players 1 and 
2 have $a$ on top, player 3 has $a$ at second position, and player 4 at the last position. This gives rise to two issues $a_{1}$ and $a_{2}$ with orderings respectively $1,2,3,4$ and $2,1,3,4$. Similarly, $b$ gives rise to four issues and $c$ results in six issues. The resulting issue profile is given in the following table:

\begin{tabular}{cccccccccccc}
\hline$a_{1}$ & $a_{2}$ & $b_{1}$ & $b_{2}$ & $b_{3}$ & $b_{4}$ & $c_{1}$ & $c_{2}$ & $c_{3}$ & $c_{4}$ & $c_{5}$ & $c_{6}$ \\
\hline 1 & 2 & 3 & 3 & 4 & 4 & 4 & 4 & 4 & 4 & 4 & 4 \\
2 & 1 & 4 & 4 & 3 & 3 & 1 & 1 & 2 & 2 & 3 & 3 \\
3 & 3 & 1 & 2 & 1 & 2 & 2 & 3 & 1 & 3 & 1 & 2 \\
4 & 4 & 2 & 1 & 2 & 1 & 3 & 2 & 3 & 1 & 2 & 1 \\
\hline
\end{tabular}

Suppose in the simple game the minimal winning coalitions are those with three players. Then $\Phi$ would assign to the resulting game $G$ the power distribution $\frac{1}{12}(4,4,4,0)=\frac{1}{18}(6,6,6,0)$. With weight vector

$$
w=\frac{1}{36}(6,6,3,3,3,3,2,2,2,2,2,2),
$$

which results from assigning equal importance to the original alternatives $a$, $b$, and $c$, we obtain $\Phi^{w}(G)=\frac{1}{18}(5,5,8,0)$.

As is the case in this example, if the number of alternatives is small relative to the number of players, then often two or more players rank the same alternative at the same position, resulting in many issues. This does not occur in the Owen-Shapley model where the number of alternatives is infinite, namely the unit circle, and thus the set of issues, obtained by the above procedure, coincides with the number of alternatives up to a set of measure zero.

While this potential abundance of issues, resulting from preferences of players over alternatives, is an important practical reason to take linear orders over players as a primitive in the model, it is certainly not the only reason. Deriving issue profiles as in Example 2.4 presupposes that these preferences are comparable, but this by itself is a strong assumption. This problem is avoided by taking issue profiles as a primitive. Moreover, in practice it seems often easier to determine an issue profile than to assess players' preferences over alternatives. For instance, concerning an issue on which a political parliament has to decide, it is usually not difficult to establish an order in which 
the parties are enthusiastic about and thus support the issue; on the other hand, it may be quite senseless to talk about preferences of parties over (possibly quite) different issues. 


\section{Chapter 3}

\section{Power indices for matching problems}

\section{1 introduction}

In this chapter, which is based on work in progress, we propose two families of power indices for so-called marriage problems in Gale and Shapley [25] and in Roth and Sotomayor [55]. In a marriage problem, there is a number of men and an equal number of women. Each man has a preference over the women, including the option of staying single, and the same holds for each woman. These preferences determine their chances on the marriage market, and in this situation a power index is meant to capture this. We approach this question by considering so-called effectivity functions, which reflect the possibilities of the men and women in a marriage problem. We take two different approaches. In the first a pproach we start from the situation where everybody is still single. Then, to any matching, i.e., map saying which pairs of men and women are formed, the others staying single, the effectivity function associated with the profile of preferences assigns those couples in the matching that prefer each other over staying single. In the second approach, again given the profile of preferences, the effectivity function assigns to every

Adapted from: 'Kong Q. and Peters H.: Power indices for matching problems. In progress, 2020'. This chapter has benefited from discussions with Dominik Karos. 
matching those couples or individuals that can block the matching. As usual, a couple can block if the man and woman in the couple prefer each other over their current partner or being single, and a man or woman can block if he or she prefers to be single over being coupled to his or her partner. In both approaches, a power index acts on the associated effectivity functions, rather than directly on the preference profile. In each case, we propose a number of axioms that characterize families of power indices. The common and central axiom is the Transfer Property (see also Chapter 2). This says that if we have two effectivity functions, then the sum of the powers assigned to these equals the sum of the powers assigned to their union and to their intersection, where these operations are defined matching-wise. After prelimaries in Section 3.2, we present our two approaches in Sections 3.3 and 3.4. Almost all proofs are relegated to appendices.

\subsection{Preliminaries}

Notations For a set $X, P(X)=\{Y: Y \subseteq X\}$. By $|X|$ we denote the number of elements of $X$.

\subsubsection{Matching problems}

We consider two-sided one-to-one matching problems, also called marriage problems. A marriage problem is a triple $(M, W, P)$, where:

- $M$ and $W$ are the disjoint sets of men and women, respectively, with $|M|=|W|=k$ for some $k \in \mathbb{N}$ with $k \geq 2$,

- $P=\left(P_{\ell}\right)_{\ell \in M \cup W}$, such that for each $m \in M, P_{m}$ is a linear order over $W \cup\{s\}$ and for each $w \in W, P_{w}$ is a linear order over $M \cup\{s\}$, where $s \notin M \cup W$ stands for being single.

We also write $N=M \cup W$ and $|N|=n$, hence $n=2 k$. Whenever confusion is unlikely to arise, we denote a marriage problem $(M, W, P)$ simply by $P$. By $\mathscr{P}$ we denote the set of all marriage problems. 
A matching is a map $\mu: N \rightarrow N \cup\{s\}$ such that (i) $\mu(m) \in W \cup\{s\}$ for all $m \in M$, (ii) $\mu(w) \in M \cup\{s\}$ for all $w \in W$, and (iii) $\mu(m)=w \Leftrightarrow \mu(w)=m$ for all $m \in M$ and $w \in W$. Let $\mathscr{M}$ denote the set of all matchings.

The purpose of this chapter is to develop 'power indices' for marriage problems, to be interpreted as reflecting the possibilities or opportunities of the men and women in such problems. Basically, a power index should reflect the possibilities of changing a matching, given one's own ranking of the members of the opposite sex, one's position in the rankings of the members of the opposite sex, and the chances of the members of the opposite sex in light of the rankings of one's competitors of the same sex. Typically, in any matching, either individuals can enforce a change by leaving their current partner and becoming single; or pairs can enforce a change, by marrying while divorcing their current partner or leaving their state as a single individual.

We will develop such power indices, not for marriage problems directly, but rather for so-called 'effectivity functions', based on marriage problems and capturing the opportunities of individuals as described above. We take two different approaches, to be introduced in the next two subsections. First, it will be convenient to also have the following definition.

Definition 3.1. A general effectivity function is a map $E: \mathscr{M} \rightarrow P((M \cup\{s\}) \times$ $(W \cup\{s\}) \backslash\{(s, s)\})$. By $\mathscr{E}$ we denote the set of all such functions.

In words, a general effectivity function assigns to each matching a (possibly empty) set of individuals and pairs. The interpretation is that these pairs and individuals are able to influence, in one way or another, the matching under consideration.

\subsubsection{Effectivity functions based on being single}

In this approach, for any marriage problem, we take the situation where every one is single as a point of departure. Then we say that a pair $(m, w) \in M \times W$ is effective at a matching $\mu \in \mathscr{M}$ if $m$ and $w$ are matched at $\mu, m$ prefers $w$ over being single, and $w$ prefers $m$ over being single. Formally, for every $P \in \mathscr{P}$ we define the mapping $E_{s}^{P}: \mathscr{M} \rightarrow P(M \times W)$ by

$$
E_{s}^{P}(\mu)=\left\{(m, w) \in M \times W: m P_{w} s, w P_{m} s, \mu(m)=w\right\}
$$


for every $\mu \in \mathscr{M}$. We denote the set of all these mappings by $\mathscr{E}_{s}$, i.e., $\mathscr{E}_{s}=$ $\left\{E_{s}^{P}: P \in \mathscr{P}, E_{s}^{P}\right.$ satisfies (3.1) $\}$. Clearly, $\mathscr{E}_{s} \subseteq \mathscr{E}$.

\subsubsection{Effectivity functions based on blocking coalitions}

In the second approach, given a marriage problem and a matching, we consider the single individuals and pairs that can 'block' $\mu$ : a single individual can block $\mu$ if he or she prefers to be single over his or her partner at $\mu$; and a pair of a man and a woman can block $\mu$ if they prefer each other over their partners (including $s$ ) at $\mu$. Formally, for every $P \in \mathscr{P}$ we define the mapping $E_{b}^{P}: \mathscr{M} \rightarrow P\left(\left\{\left(\ell, \ell^{\prime}\right): \ell \in M \cup\{s\}, \ell^{\prime} \in W \cup\{s\},\left(\ell, \ell^{\prime}\right) \neq(s, s)\right\}\right)$ by

$$
\begin{aligned}
E_{b}^{P}(\mu)= & \left\{(m, w) \in M \times W: w P_{m} \mu(m), m P_{w} \mu(w)\right\} \\
& \cup\left\{(m, s) \in M \times\{s\}: s P_{m} \mu(m)\right\} \\
& \cup\left\{(s, w) \in\{s\} \times W: s P_{w} \mu(w)\right\}
\end{aligned}
$$

for every $\mu \in \mathscr{M}$. We denote the set of all these mappings by $\mathscr{E}_{b}$, i.e., $\mathscr{E}_{b}=$ $\left\{E_{b}^{P}: P \in \mathscr{P}, E_{b}^{P}\right.$ satisfies (3.2) $\}$. Clearly, $\mathscr{E}_{b} \subseteq \mathscr{E}$.

\subsubsection{Power indices and the Transfer Property}

Let $\mathscr{E}^{\prime}$ be a subset of $\mathscr{E}$.

Definition 3.2. A power index on $\mathscr{E}^{\prime}$ is a map $\varphi: \mathscr{E}^{\prime} \rightarrow \mathbb{R}^{N}$ with $\sum_{\ell \in N} \varphi_{\ell}(E)=$ 0 for every $E \in \mathscr{E}^{\prime}$.

The implicit condition that total power is zero, is a way of scaling power. In particular, we allow power to be negative. As a somewhat loose interpretation, positive power reflects that opportunities outweigh threats on the marriage market, while negative power reflects the opposite. Cf. Karos and Peters [37], who apply a similar scaling.

An axiom for power indices that will be used throughout, is the equivalent of the Transfer Property for power indices on simple games, introduced by Dubey [17]. In order to formulate this property, for all $E^{1}, E^{2} \in \mathscr{E}$, we define 
$E^{1} \cup E^{2} \in \mathscr{E}$ and $E^{1} \cap E^{2} \in \mathscr{E}$ by

$$
\begin{aligned}
& \left(E^{1} \cup E^{2}\right)(\mu)=E^{1}(\mu) \cup E^{2}(\mu) \\
& \left(E^{1} \cap E^{2}\right)(\mu)=E^{1}(\mu) \cap E^{2}(\mu)
\end{aligned}
$$

for all $\mu \in \mathscr{M}$.

Let $\varphi$ be a power index on $\mathscr{E}^{\prime} \subseteq \mathscr{E}$ and assume that $\mathscr{E}^{\prime}$ is closed under union and intersection.

Transfer Property $\varphi\left(E^{1} \cup E^{2}\right)+\varphi\left(E^{1} \cap E^{2}\right)=\varphi\left(E^{1}\right)+\varphi\left(E^{2}\right)$ for all $E^{1}, E^{2} \in \mathscr{E}^{\prime}$.

\subsection{Power in the singles market}

In this section we develop a class of power indices $\varphi: \mathscr{E}_{s} \rightarrow \mathbb{R}^{N}$, based on the situation where every individual is single - see Section 3.2.2. Hence, with each marriage problem $P \in \mathscr{P}$ we associate a vector $\varphi\left(E_{s}^{P}\right)$, expressing each individual's chances in the marriage market when nobody is married yet. Throughout this section we write $E^{P}$ instead of $E_{s}^{P}$ for every $P \in \mathscr{P}$.

We introduce some further notation. For $w \in W$ and $P \in \mathscr{P}$, we denote by $U(P, w)=\left\{m \in M: m P_{w} s\right\}$ and $U(P, m)=\left\{w \in W: w P_{m} s\right\}$ the sets of men and women, respectively, with whom $w$ and $m$ would like to marry. Further, for $P \in \mathscr{P}, T(P)=\left\{(m, w) \in M \times W: w P_{m} s, m P_{w} s\right\}$ denotes the set of potential pairs.

We first show that the set $\mathscr{E}_{S}$ is closed under the union and intersection operators defined above.

Lemma 3.1. Let $P, Q \in \mathscr{P}$. Then $E^{P} \cup E^{Q} \in \mathscr{E}_{s}$ and $E^{P} \cap E^{Q} \in \mathscr{E}_{s}$.

Proof. Let $R \in \mathscr{P}$ such that $U(R, m)=\{w \in W:(m, w) \in T(P) \cup T(Q)\}$ for all $m \in M$ and $U(R, w)=\{m \in M:(m, w) \in T(P) \cup T(Q)\}$ for all $w \in W$. Then for each $\mu \in \mathscr{M}$ and $(m, w) \in M \times W$ we have $(m, w) \in E^{R}(\mu)$ if and only if $(m, w) \in E^{P}(\mu) \cup E^{Q}(\mu)$. Thus, $E^{R}=E^{P} \cup E^{Q}$.

Let $R^{\prime} \in \mathscr{P}$ such that $U\left(R^{\prime}, m\right)=\{w \in W:(m, w) \in T(P) \cap T(Q)\}$ for all $m \in M$ and $U\left(R^{\prime}, w\right)=\{m \in M:(m, w) \in T(P) \cap T(Q)\}$ for all $w \in W$. Then, similarly, $E^{R^{\prime}}=E^{P} \cap E^{Q}$. 
The following example illustrates Lemma 3.1 and its proof.

Example 3.1. Consider $P, Q \in \mathscr{P}$ in Tables 3.1 and 3.2. Table 3.3 exhibits $R$ with $E^{R}=E^{P} \cup E^{Q}$, and Table 3.4 exhibits $R^{\prime}$ with $E^{R^{\prime}}=E^{P} \cap E^{Q}$, as in the proof of Lemma 3.1.

TABLE 3.1: Marriage problem $P$

\begin{tabular}{cccccc}
\hline$m_{1}$ & $m_{2}$ & $m_{3}$ & $w_{1}$ & $w_{2}$ & $w_{3}$ \\
\hline$w_{1}$ & $w_{1}$ & $w_{1}$ & $m_{1}$ & $m_{1}$ & $m_{1}$ \\
$w_{2}$ & $w_{3}$ & $w_{2}$ & $m_{2}$ & $m_{2}$ & $m_{3}$ \\
$s$ & $s$ & $w_{3}$ & $s$ & $s$ & $s$ \\
$w_{3}$ & $w_{2}$ & $s$ & $m_{3}$ & $m_{3}$ & $m_{2}$ \\
\hline
\end{tabular}

TABLE 3.3: Marriage problem $R$

\begin{tabular}{cccccc}
\hline$m_{1}$ & $m_{2}$ & $m_{3}$ & $w_{1}$ & $w_{2}$ & $w_{3}$ \\
\hline$w_{1}$ & $w_{1}$ & $w_{3}$ & $m_{1}$ & $m_{1}$ & $m_{3}$ \\
$w_{2}$ & $w_{2}$ & $s$ & $m_{2}$ & $m_{2}$ & $s$ \\
$s$ & $s$ & $w_{1}$ & $s$ & $s$ & $m_{1}$ \\
$w_{3}$ & $w_{3}$ & $w_{2}$ & $m_{3}$ & $m_{3}$ & $m_{2}$ \\
\hline
\end{tabular}

TABLE 3.2: Marriage problem $Q$

\begin{tabular}{cccccc}
\hline$m_{1}$ & $m_{2}$ & $m_{3}$ & $w_{1}$ & $w_{2}$ & $w_{3}$ \\
\hline$w_{1}$ & $w_{1}$ & $w_{1}$ & $m_{1}$ & $m_{2}$ & $m_{1}$ \\
$w_{2}$ & $w_{2}$ & $w_{3}$ & $m_{2}$ & $m_{3}$ & $s$ \\
$s$ & $s$ & $s$ & $s$ & $s$ & $m_{2}$ \\
$w_{3}$ & $w_{3}$ & $w_{2}$ & $m_{3}$ & $m_{1}$ & $m_{3}$ \\
\hline
\end{tabular}

TABLE 3.4: Marriage problem $R^{\prime}$

\begin{tabular}{cccccc}
\hline$m_{1}$ & $m_{2}$ & $m_{3}$ & $w_{1}$ & $w_{2}$ & $w_{3}$ \\
\hline$w_{1}$ & $w_{1}$ & $s$ & $m_{1}$ & $s$ & $s$ \\
$s$ & $s$ & $w_{1}$ & $m_{2}$ & $m_{1}$ & $m_{1}$ \\
$w_{2}$ & $w_{2}$ & $w_{2}$ & $s$ & $m_{2}$ & $m_{2}$ \\
$w_{3}$ & $w_{3}$ & $w_{3}$ & $m_{3}$ & $m_{3}$ & $m_{3}$ \\
\hline & & & & & $\triangleleft$
\end{tabular}

Besides the Transfer Property introduced in Section 3.2.4, we impose two other conditions on a power index $\varphi$ defined on $\mathscr{E}_{s}$. To define the first one, symmetry, let $P \in \mathscr{P}$. We call two individuals $\ell, \ell^{\prime} \in N$ symmetric in $E^{P}$ if one of the following (mutually exclusive) conditions is satisfied:

(i) for all $\mu \in \mathscr{M}$ and all $(m, w) \in E^{P}(\mu)$, we have $\ell, \ell^{\prime} \notin\{m, w\}$, or

(ii) for all $(m, w) \in T(P)$, we have $\ell=m$ if and only if $\ell^{\prime}=w$.

Condition (i) says that all individuals who are never a member of any pair that prefers to be married over staying single, are regarded as symmetric - in 
fact, they could all be regarded as dummies in the singles market. Condition (ii) covers, in some sense, the other extreme: if $\ell$ and $\ell^{\prime}$ can only marry each other, then they should be regarded as symmetric.

Symmetry $\varphi_{\ell}\left(E^{P}\right)=\varphi_{\ell^{\prime}}\left(E^{P}\right)$ for all $P \in \mathscr{P}$ and all $\ell, \ell^{\prime} \in N$ who are symmetric in $E^{P}$.

The second additional property requires, ceteris paribus, that if an individual $\ell_{2}$ first wants to marry $\ell_{1}$, but later changes his/her mind and prefers to be single over marrying $\ell_{1}$, then $\ell_{1}$ 's power decreases.

Monotonicity $\varphi_{\ell_{1}}\left(E^{P^{\prime}}\right) \leq \varphi_{\ell_{1}}\left(E^{P}\right)$ for all $P, P^{\prime} \in \mathscr{P}$ and $\ell_{1}, \ell_{2} \in N$ such that $\ell_{1} \in U\left(P, \ell_{2}\right), U\left(P^{\prime}, \ell\right)=U(P, \ell)$ for all $\ell \neq \ell_{2}$, and $U\left(P^{\prime}, \ell_{2}\right)=U\left(P, \ell_{2}\right) \backslash\left\{\ell_{1}\right\}$.

The main result in this section will show that power indices on $\mathscr{E}_{S}$ satisfying the Transfer Property, Symmetry, and Monotonicity are determined by weights attached to pairs. A weight system (on $M \times W$ ) is a collection of nonnegative real numbers $\omega=\left(\omega^{m, w}\right)_{m \in M, w \in W}$. For a weight system $\omega$ we define the mapping $\Phi^{\omega}: \mathscr{E}_{s} \rightarrow \mathbb{R}^{N}$ as follows. For all $P \in \mathscr{P}$,

$$
\begin{aligned}
& \Phi_{m}^{\omega}\left(E^{P}\right)=\sum_{w:(m, w) \in T(P)} \omega^{m, w}-\sum_{m^{\prime} \neq m, w:\left(m^{\prime}, w\right) \in T(P)} \frac{2 \omega^{m^{\prime}, w}}{2 k-2} \text { for every } m \in M, \\
& \Phi_{w}^{\omega}\left(E^{P}\right)=\sum_{m:(m, w) \in T(P)} \omega^{m, w}-\sum_{w^{\prime} \neq w, m:\left(m, w^{\prime}\right) \in T(P)} \frac{2 \omega^{m, w^{\prime}}}{2 k-2} \text { for every } w \in W .
\end{aligned}
$$

Thus, according to $\Phi^{\omega}$, in a marriage problem $P$, every man receives the weights from all potential pairs involving him, minus his share of the weights that accrue to the potential pairs not involving him. An analogous explanation, of course, holds for every woman.

The main result of this section is as follows. See Appendix A for its proof.

Theorem 3.2. Let $\varphi$ be a power index on $\mathscr{E}_{s}$. Then $\varphi$ satisfies the Transfer Property, Symmetry, and Monotonicity, if and only if $\varphi=\Phi^{\omega}$ for a weight system $\omega$.

We conclude this section with a few examples. The first example shows that the constant power index is a special case. 
Example 3.2. Let $\omega^{m, w}=0$ for all $(m, w) \in M \times W$. For this choice of weights, we obtain the constant power index, i.e., $\Phi_{\ell}^{\omega}\left(E^{P}\right)=0$ for every $P \in \mathscr{P}$ and $\ell \in N$.

The second example shows a case when all powers are equal, and hence zero.

Example 3.3. For $P \in \mathscr{P}$ and $m \in M$ define $\gamma_{m}=|\{w \in W:(m, w) \in T(P)\}|$. Similarly, for $w \in W, \gamma_{w}=|\{m \in M:(m, w) \in T(P)\}|$.

Let $\omega$ be a weight system with $\omega^{m, w}=\omega^{m^{\prime}, w^{\prime}}$ for all $m, m^{\prime} \in M$ and $w, w^{\prime} \in W$. If, for some marriage problem $P, \gamma_{\ell_{1}}=\gamma_{\ell_{2}}$ for all $\ell_{1}, \ell_{2} \in N$, we have $\Phi_{\ell}^{\omega}\left(E^{P}\right)=0$ for every $\ell \in N$. For instance, consider the following matching problem. We have $T(P)=\left\{\left(m_{1}, w_{1}\right),\left(m_{1}, w_{2}\right),\left(m_{2}, w_{1}\right),\left(m_{2}, w_{2}\right)\right\}$

TABLE 3.5 Matching problem $P$.

\begin{tabular}{cccc}
\hline$m_{1}$ & $m_{2}$ & $w_{1}$ & $w_{2}$ \\
\hline$w_{1}$ & $w_{1}$ & $m_{1}$ & $m_{1}$ \\
$w_{2}$ & $w_{2}$ & $m_{2}$ & $m_{2}$ \\
$s$ & $s$ & $s$ & $s$ \\
\hline
\end{tabular}

and hence $\gamma_{\ell}=2$ for every $\ell \in N$. Then $\Phi_{\ell}^{\omega}\left(E^{P}\right)=0$ for every $\ell \in N$.

\subsection{Power by blocking}

Here we study power in the marriage problem via effectivity functions in $\mathscr{E}_{b}$, as defined in Section 3.2.3, in particular by (3.2). Henceforth, in this section, we write $E^{P}$ instead of $E_{b}^{P}$, for $P \in \mathscr{P}$ and $E_{b}^{P} \in \mathscr{E}_{b}$. Just to repeat, such an effectivity function provides, for every matching $\mu$, the pairs and singles that can block $\mu$, given their preferences.

One difficulty is that the set $\mathscr{E}_{b}$ is not closed under union and intersection: there does not have to be an $R \in \mathscr{P}$ such that for $P, Q \in \mathscr{P}$, we have $E^{R}=E^{P} \cup E^{Q}$, or $E^{R}=E^{P} \cap E^{Q}$. This is a potential problem when applying the Transfer Property for a power index. Moreover, in our arguments we will need certain effectivity functions which are, again, not of the form $E^{R}$ for 
some $R \in \mathscr{P}$. We will solve these problems by defining a larger set of effectivity functions, containing the set $\mathscr{E}_{b}$, and contained in the set $\mathscr{E}$ of general effectivity functions as in Definition 3.1.

For every pair $(m, w) \in M \times W$ and matching $\mu \in \mathscr{M}$ define, for every $\mu^{\prime} \in \mathscr{M}$,

$$
E^{m, w, \mu}\left(\mu^{\prime}\right)= \begin{cases}\{(m, w)\} & \text { if } \mu^{\prime}=\mu \text { and } \mu(m) \neq w \\ \emptyset & \text { otherwise. }\end{cases}
$$

For every $m \in M$ and matching $\mu \in \mathscr{M}$ define, for every $\mu^{\prime} \in \mathscr{M}$,

$$
E^{m, \mu}\left(\mu^{\prime}\right)= \begin{cases}\{(m, s)\} & \text { if } \mu^{\prime}=\mu \text { and } \mu(m) \neq s \\ \emptyset & \text { otherwise. }\end{cases}
$$

For every $w \in W$ and matching $\mu \in \mathscr{M}$ define, for every $\mu^{\prime} \in \mathscr{M}$,

$$
E^{w, \mu}\left(\mu^{\prime}\right)= \begin{cases}\{(s, w)\} & \text { if } \mu^{\prime}=\mu \text { and } \mu(w) \neq s \\ \emptyset & \text { otherwise. }\end{cases}
$$

Finally, we denote by $E^{\emptyset}$ the empty effectivity function, i.e., $E^{\emptyset}(\mu)=\emptyset$ for all $\mu \in \mathscr{M}$. For instance, $E^{m, w, \mu}\left(\mu^{\prime}\right)=\emptyset$ for all $\mu^{\prime} \in \mathscr{M}$ whenever $\mu(m)=w$.

We now define the set $\mathscr{E}_{*}$ as the set of all unions and intersections of elements of the set

$$
\left\{E^{P}, E^{m, w, \mu}, E^{\ell, \mu}: P \in \mathscr{P},(m, w) \in M \times W, \ell \in N, \mu \in \mathscr{M}\right\} .
$$

Clearly, $\mathscr{E}_{b} \subseteq \mathscr{E}_{*} \subseteq \mathscr{E}$. In this section, any power index $\varphi$ is assumed to be defined on $\mathscr{E}_{*}$. Besides the Transfer Property, we will again consider an axiom of symmetry.

Let $E \in \mathscr{E}_{*}$ and $\ell_{1}, \ell_{2} \in N$. Then $\ell_{1}$ and $\ell_{2}$ are symmetric in $E$ if one of the following (mutually exclusive) conditions is satisfied:

(i) for every $\mu \in \mathscr{M}$ and every $\left(\ell, \ell^{\prime}\right) \in(M \cup\{s\}) \times(W \cup\{s\})$ such that $\left(\ell, \ell^{\prime}\right) \in E(\mu)$, we have $\left\{\ell_{1}, \ell_{2}\right\} \cap\left\{\ell, \ell^{\prime}\right\}=\emptyset$, or 
(ii) for all $(m, w) \in M \times W$ and every $\mu \in \mathscr{M}$ such that $(m, w) \in E(\mu)$, we have $\ell_{1}=m$ if and only if $\ell_{2}=w$.

Observe that these cases are similar to the analogous cases in Section 3.3.

Symmetry $\varphi_{\ell}(E)=\varphi_{\ell^{\prime}}(E)$ for all $\ell, \ell^{\prime} \in N$ who are symmetric in $E$.

The next property says that the power of an individual who can never block, alone or in a pair, cannot be larger than the power of any other individual. Call $\ell \in N$ a dummy in $E \in \mathscr{E}_{*}$ if $\ell \notin\left\{\ell_{1}, \ell_{2}\right\}$ for every $\mu \in \mathscr{M}$ and every $\left(\ell_{1}, \ell_{2}\right) \in E(\mu)$.

Dummy Property $\varphi_{\ell}(E) \leq \varphi_{\ell^{\prime}}(E)$ for all $E \in \mathscr{E}_{*}$, every $\ell \in N$ who is a dummy in $E$, and every $\ell^{\prime} \in N \backslash\{\ell\}$.

The final property says that, if two effectivity functions are equal up to a permutation of matchings, then for every individual his or her power in the two effectivity functions should be equal.

Matching Neutrality $\varphi_{\ell}(E)=\varphi_{\ell}\left(E^{\prime}\right)$ for all $\ell \in N$ and $E, E^{\prime} \in \mathscr{E}_{*}$ for which there is a permutation $\pi: \mathscr{M} \rightarrow \mathscr{M}$ such that $E(\mu)=E^{\prime}(\pi(\mu))$ for all $\mu \in \mathscr{M}$.

A weight system on $(M \cup\{s\} \times W \cup\{s\}) \backslash\{(s, s)\}$ is a collection of nonnegative real numbers $\tau=\left(\left(\tau^{m, w}\right)_{m \in M, w \in W},\left(\tau^{\ell}\right)_{\ell \in N}\right)$. For a weight system $\tau$ we define the mapping $\Psi^{\tau}: \mathscr{E}_{*} \rightarrow \mathbb{R}^{N}$ by

$$
\begin{aligned}
\Psi_{m}^{\tau}(E)= & \sum_{\substack{w, \mu: \\
(m, w) \in E(\mu)}} \tau^{m, w}+\sum_{\mu:(m, s) \in E(\mu)} \tau^{m}-\sum_{\substack{m^{\prime} \neq m, w, \mu \\
\left(m^{\prime}, w\right) \in E(\mu)}} \frac{2 \tau^{m^{\prime}, w}}{n-2} \\
& -\sum_{\substack{m^{\prime} \neq m, \mu: \\
\left(m^{\prime}, s\right) \in E(\mu)}} \frac{\tau^{m^{\prime}}}{n-1}-\sum_{\substack{w, \mu: \\
(s, w) \in E(\mu)}} \frac{\tau^{w}}{n-1} \\
\Psi_{w}^{\tau}(E)= & \sum_{\substack{m, \mu: \\
(m, w) \in E(\mu)}} \tau^{m, w}+\sum_{\mu:(s, w) \in E(\mu)} \tau^{w}-\sum_{\substack{m, w^{\prime} \neq w, \mu: \\
\left(m, w^{\prime}\right) \in E(\mu)}} \frac{2 \tau^{m, w^{\prime}}}{n-2} \\
& -\sum_{\substack{w^{\prime} \neq w, \mu: \\
\left(s, w^{\prime}\right) \in E(\mu)}} \frac{\tau^{w^{\prime}}}{n-1}-\sum_{\substack{m, \mu: \\
(m, s) E(\mu)}} \frac{\tau^{m}}{n-1}
\end{aligned}
$$


for every $m \in M$, every $w \in W$ and every $E \in \mathscr{E}_{*}$. Thus, every $m \in M$ receives a weight from every matching and every woman with whom he can block that matching, plus a weight from every matching in which $m$ can block by divorcing, minus a share of the weights that accrue to every matching in which some other pair can block, minus a share of the weights from every matching in which some other man or woman can block. An analogous explanation holds for the payoff of every $w \in W$.

The main result of this section is as follows. See Appendix $C$ for its proof.

Theorem 3.3. Let $\varphi$ be a power index on $\mathscr{E}_{*}$. Then $\varphi$ satisfies the Transfer Property, Symmetry, the Dummy Property, and Matching Neutrality if and only if there is a weight system $\tau$ such that $\varphi=\Psi^{\tau}$.

We conclude with a few examples, similar to those in Section 3.3.

Example 3.4. Let $\tau^{m, w}=\tau^{m}=\tau^{w}=0$ for all $m \in M$ and $w \in W$. For this choice of weights, we obtain the constant power index, i.e., $\Psi_{\ell}^{\tau}(E)=0$ for every $E \in \mathscr{E}_{*}$ and $\ell \in N$.

Example 3.5. Let $\tau$ be a weight system such that $\tau^{m, w}=\tau^{m^{\prime}, w^{\prime}}$ for all $m, m^{\prime} \in$ $M, w, w^{\prime} \in W$, and $\tau^{\ell}=\tau^{\ell^{\prime}}$ for all $\ell, \ell^{\prime} \in N$. Suppose that, for some $E \in \mathscr{E}_{*}$, we have $\left|\left\{w^{\prime} \in W, \mu \in \mathscr{M}:\left(m, w^{\prime}\right) \in E(\mu)\right\}\right|=\mid\left\{w^{\prime} \in W, \mu \in \mathscr{M}:\left(\widetilde{m}, w^{\prime}\right) \in\right.$ $E(\mu)\}|=|\left\{m^{\prime} \in M, \mu \in \mathscr{M}:\left(m^{\prime}, w\right) \in E(\mu)\right\}|=|\left\{m^{\prime} \in M, \mu \in \mathscr{M}:\left(m^{\prime}, \widetilde{w}\right) \in\right.$ $E(\mu)\} \mid$ and that $|\{\mu \in \mathscr{M}:(m, s) \in E(\mu)\}|=|\{\mu \in \mathscr{M}:(\tilde{m}, s) \in E(\mu)\}|=$ $|\{\mu \in \mathscr{M}:(s, w) \in E(\mu)\}|=|\{\mu \in \mathscr{M}:(s, \widetilde{w}) \in E(\mu)\}|$ for all $m, \widetilde{m} \in M$ and $w, \widetilde{w} \in W$. Then we have $\Psi_{\ell}^{\tau}(E)=0$ for every $\ell \in N$. For instance, consider the following matching problem $P$. The corresponding effectivity function satisfies the conditions above, and $\Psi_{\ell}^{\tau}(E)=0$ for every $\ell \in N$.

\begin{tabular}{cccc}
\hline$m_{1}$ & $m_{2}$ & $w_{1}$ & $w_{2}$ \\
\hline$s$ & $s$ & $s$ & $s$ \\
$w_{1}$ & $w_{2}$ & $m_{1}$ & $m_{2}$ \\
$w_{2}$ & $w_{1}$ & $m_{2}$ & $m_{1}$ \\
\hline
\end{tabular}


The following example shows that the monotonicity condition in its form as in Section 3.3 does not have to hold for a power index of the form $\Psi^{\tau}$.

Example 3.6. Consider the matching problem $P$ in Table 3.5 and the matching problem $Q$ in Table 3.6.

TABLE 3.6 Matching problem $Q$.

\begin{tabular}{cccc}
\hline$m_{1}$ & $m_{2}$ & $w_{1}$ & $w_{2}$ \\
\hline$w_{2}$ & $w_{2}$ & $m_{2}$ & $m_{1}$ \\
$w_{1}$ & $w_{1}$ & $s$ & $m_{2}$ \\
$s$ & $s$ & $m_{1}$ & $s$ \\
\hline
\end{tabular}

Clearly, $U\left(Q, m_{1}\right)=U\left(P, m_{1}\right), U\left(Q, m_{2}\right)=U\left(P, m_{2}\right), U\left(Q, w_{2}\right)=U\left(P, w_{2}\right)$ and $U\left(Q, w_{1}\right)=U\left(P, w_{1}\right) \backslash\left\{m_{1}\right\}$. However, if $2 \tau^{m_{1}, w_{2}}>5 \tau^{m_{1}, w_{1}}+\frac{2}{3} \tau^{w_{1}}+$ $\tau^{m_{2}, w_{2}}$, then $\Psi_{m_{1}}^{\tau}\left(E_{b}^{P}\right)<\Psi_{m_{1}}^{\tau}\left(E_{b}^{Q}\right)$.

\section{Appendix A Remaining proofs of Section 3.3}

The proof of Theorem 3.2 is based on a number of lemmas. In the next three lemmas, $\omega$ is a nonnegative weight system.

Lemma 3.4. $\Phi^{\omega}$ satisfies the Transfer Property.

Proof. Let $P^{1}, P^{2} \in \mathscr{P}$. Then by the proof of Lemma 3.1 we have $E^{P_{1}} \cup E^{P_{2}}=$ $E^{R}$ and $E^{P_{1}} \cap E^{P_{2}}=E^{R^{\prime}}$, where $R \in \mathscr{P}$ such that $U(R, m)=\{w \in W:(m, w) \in$ $T(P) \cup T(Q)\}$ for all $m \in M$ and $U(R, w)=\{m \in M:(m, w) \in T(P) \cup T(Q)\}$ for all $w \in W$; and $R^{\prime} \in \mathscr{P}$ such that $U\left(R^{\prime}, m\right)=\{w \in W:(m, w) \in T(P) \cap T(Q)\}$ for all $m \in M$ and $U\left(R^{\prime}, w\right)=\{m \in M:(m, w) \in T(P) \cap T(Q)\}$ for all $w \in W$.

Now, for every $m \in M$, we have

$$
\Phi_{m}^{\omega}\left(E^{P^{1}} \cup E^{P^{2}}\right)=\sum_{w:(m, w) \in T\left(P^{1}\right) \cup T\left(P^{2}\right)} \omega^{m, w}-\sum_{m^{\prime} \neq m, w:\left(m^{\prime}, w\right) \in T\left(P^{1}\right) \cup T\left(P^{2}\right)} \frac{2 \omega^{m^{\prime}, w}}{2 k-2}
$$


and

$$
\Phi_{m}^{\omega}\left(E^{P^{1}} \cap E^{P^{2}}\right)=\sum_{w:(m, w) \in T\left(P^{1}\right) \cap T\left(P^{2}\right)} \omega^{m, w}-\sum_{m^{\prime} \neq m, w:\left(m^{\prime}, w\right) \in T\left(P^{1}\right) \cap T\left(P^{2}\right)} \frac{2 \omega^{m^{\prime}, w}}{2 k-2}
$$

from which it easily follows that $\Phi_{m}^{\omega}\left(E^{P^{1}} \cup E^{P^{2}}\right)+\Phi_{m}^{\omega}\left(E^{P^{1}} \cap E^{P^{2}}\right)=\Phi_{m}^{\omega}\left(E^{P^{1}}\right)+$ $\Phi_{m}^{\omega}\left(E^{P^{2}}\right)$. The argument for $w \in W$ is analogous.

Lemma 3.5. $\Phi^{\omega}$ satisfies Symmetry.

Proof. Let $P \in \mathscr{P}$. First, suppose that $\ell, \ell^{\prime} \in M \cup W$ are symmetric players in $E^{P}$ according to (i) in the definition of symmetric players. Then for every $(m, w) \in T(P)$ we have $\left\{\ell, \ell^{\prime}\right\} \cap\{m, w\}=\emptyset$. If $\ell \in M$, this implies that

$$
\Phi_{\ell}^{\omega}\left(E^{P}\right)=-\sum_{m \neq \ell, w:(m, w) \in T(P)} \frac{2 \omega^{m, w}}{2 k-2},
$$

and if $\ell \in W$, then

$$
\Phi_{\ell}^{\omega}\left(E^{P}\right)=-\sum_{w \neq \ell, m:(m, w) \in T(P)} \frac{2 \omega^{m, w}}{2 k-2} .
$$

Similarly, If $\ell^{\prime} \in M$, then

$$
\Phi_{\ell^{\prime}}^{\omega}\left(E^{P}\right)=-\sum_{m \neq \ell^{\prime}, w:(m, w) \in T(P)} \frac{2 \omega^{m, w}}{2 k-2}
$$

and if $\ell^{\prime} \in W$, then

$$
\Phi_{\ell^{\prime}}^{\omega}\left(E^{P}\right)=-\sum_{w \neq \ell^{\prime}, m:(m, w) \in T(P)} \frac{2 \omega^{m, w}}{2 k-2} .
$$

Since, in these four expressions, the sums are over all $(m, w) \in T(P)$, all four expressions are equal.

Second, suppose that $\ell, \ell^{\prime} \in M \cup W$ are symmetric players in $E^{P}$ according to (ii) in the definition of symmetric players, say $\ell \in M$ and $\ell^{\prime} \in W$. Again, in the formulas for $\Phi_{\ell}^{\omega}\left(E^{P}\right)$ and $\Phi_{\ell^{\prime}}^{\omega}\left(E^{P}\right)$ all terms are equal, so that $\Phi_{\ell}^{\omega}\left(E^{P}\right)=$ $\Phi_{\ell^{\prime}}^{\omega}\left(E^{P}\right)$. 
Lemma 3.6. $\Phi^{\omega}$ satisfies Monotonicity.

Proof. Let $P, P^{\prime} \in \mathscr{P}$ and $\ell_{1}, \ell_{2} \in N$ such that $\ell_{1} \in U\left(P, \ell_{2}\right), U\left(P^{\prime}, \ell\right)=U(P, \ell)$ for all $\ell \neq \ell_{2}$, and $U\left(P^{\prime}, \ell_{2}\right)=U\left(P, \ell_{2}\right) \backslash\left\{\ell_{1}\right\}$. Suppose that $\ell_{1} \in M$ and $\ell_{2} \in W$. Clearly, $\left(\ell_{1}, \ell_{2}\right) \notin T\left(P^{\prime}\right)$. If $\left(\ell_{1}, \ell_{2}\right) \notin T(P)$ then $\Phi^{\omega}\left(E^{P}\right)=\Phi^{\omega}\left(E^{P^{\prime}}\right)$. If $\left(\ell_{1}, \ell_{2}\right) \in T(P)$, then

$$
\begin{aligned}
\Phi_{\ell_{1}}^{\omega}\left(E^{P}\right) & =\omega^{\ell_{1}, \ell_{2}}+\sum_{w \neq \ell_{2}:\left(\ell_{1}, w\right) \in T(P)} \omega^{\ell_{1}, w}-\sum_{m \neq \ell_{1}, w:(m, w) \in T(P)} \frac{2 \omega^{m, w}}{2 k-2} \\
& \geq \sum_{w \neq \ell_{2}:\left(\ell_{1}, w\right) \in T(P)} \omega^{\ell_{1}, w}-\sum_{m \neq \ell_{1}, w:(m, w) \in T(P)} \frac{2 \omega^{m, w}}{2 k-2} \\
& =\sum_{w:\left(\ell_{1}, w\right) \in T\left(P^{\prime}\right)} \omega^{\ell_{1}, w}-\sum_{m \neq \ell_{1}, w:(m, w) \in T\left(P^{\prime}\right)} \frac{2 \omega^{m, w}}{2 k-2} \\
& =\Phi_{\ell_{1}}^{\omega}\left(E^{P^{\prime}}\right),
\end{aligned}
$$

which completes the proof.

We now turn to proving the converse of Theorem 3.2.

For $P \in \mathscr{P}, m \in M$, and $w \in W$ let $P^{m, w}$ be the marriage problem defined as follows. First, let $m^{\prime} \in M$. If $w P_{m^{\prime}} s$, then $w P_{m^{\prime}}^{m, w} s, s P_{m^{\prime}}^{m, w} w^{\prime}$ for all $w^{\prime} \in$ $W \backslash\{w\}$, and $w^{\prime} P_{m^{\prime}}^{m, w} w^{\prime \prime} \Leftrightarrow w^{\prime} P_{m^{\prime}} w^{\prime \prime}$ for all $w^{\prime}, w^{\prime \prime} \in W \backslash\{w\}$. If $s P_{m^{\prime}} w$, then $s P_{m^{\prime}}^{m, w} w^{\prime}$ for all $w^{\prime} \in W$, and $w^{\prime} P_{m^{\prime}}^{m, w} w^{\prime \prime} \Leftrightarrow w^{\prime} P_{m^{\prime}} w^{\prime \prime}$ for all $w^{\prime}, w^{\prime \prime} \in W$. An analogous definition holds for $P_{w^{\prime}}^{m, w}$, for every $w^{\prime} \in W$. In words, $P^{m, w}$ is the marriage problem in which all men have woman $w$ and staying single as their top two choices, agreeing with their preferences in $P$, while for the rest their preferences are unchanged; and all women have man $m$ or staying single as their top two choices, agreeing with their preferences in $P$, while for the rest their preferences are unchanged.

Clearly, $E^{P^{m, w}}(\mu)=\{(m, w)\}$ if $(m, w) \in E^{P}(\mu)$, and $E^{P^{m, w}}(\mu)=\emptyset$ otherwise. We denote by $E^{\emptyset} \in \mathscr{E}_{s}$ the empty-valued effectivity function, i.e., $E^{\emptyset}(\mu)=\emptyset$ for every $\mu \in \mathscr{M}$. Note that for all $P \in \mathscr{P}$, we have $E^{P}=E^{\emptyset}$ if and only if $T(P)=\emptyset$.

We next show that if a power index $\varphi$ on $\mathscr{E}_{s}$ satisfies the Transfer Property, then for every $P \in \mathscr{P}, \varphi\left(E^{P}\right)$ is completely determined by the values $\varphi\left(E^{P^{m, w}}\right)$ for all $(m, w) \in M \times W$, and by $\varphi\left(E^{\emptyset}\right)$. 
Lemma 3.7. Let $P \in \mathscr{P}$. Let $\varphi$ and $\psi$ be power indices on $\mathscr{E}$ satisfying the Transfer Property, let $\varphi\left(E^{P^{m, w}}\right)=\psi\left(E^{P^{m, w}}\right)$ for all $(m, w) \in M \times W$, and let $\varphi\left(E^{\emptyset}\right)=\psi\left(E^{\emptyset}\right)$. Then $\varphi\left(E^{P}\right)=\psi\left(E^{P}\right)$.

Proof. Observe that $E^{P}=\cup_{m \in M, w \in W} E^{P^{m, w}}$. Using enumerations $M=\left\{m_{1}, \ldots, m_{k}\right\}$ and $W=\left\{w_{1}, \ldots, w_{k}\right\}$, repeated application of the Transfer Property yields

$$
\begin{aligned}
\varphi\left(E^{P}\right) & =\varphi\left(\cup_{(m, w) \in M \times W \backslash\left(m_{1}, w_{2}\right)} E^{P^{m, w}}\right)+\varphi\left(E^{P^{m_{1}, w_{1}}}\right)-\varphi\left(E^{\emptyset}\right) \\
& \vdots \\
& =\sum_{(m, w) \in M \times W} \varphi\left(E^{P^{m, w}}\right)-\left(k^{2}-1\right) \varphi\left(E^{\emptyset}\right) \\
& =\sum_{(m, w) \in M \times W} \psi\left(E^{P^{m, w}}\right)-\left(k^{2}-1\right) \psi\left(E^{\emptyset}\right) \\
& \vdots \\
& =\psi\left(\cup_{(m, w) \in M \times W \backslash\left(m_{1}, w_{2}\right)} E^{P^{m, w}}\right)+\psi\left(E^{P^{m_{1}, w_{1}}}\right)-\psi\left(E^{\emptyset}\right) \\
& =\psi\left(E^{P}\right)
\end{aligned}
$$

which proves the lemma.

We can now provide the complete proof of Theorem 3.2.

Proof of Theorem 3.2. We first prove $\Phi^{\omega}$ is a power index. Let $P \in \mathscr{P}$. Then

$$
\sum_{m \in M} \Phi_{m}^{\omega}\left(E^{P}\right)+\sum_{w \in W} \Phi_{w}^{\omega}\left(E^{P}\right)=\sum_{(m, w) \in T(P)}\left(2 \omega^{m, w}-2(k-1) \frac{2 \omega^{m, w}}{2 k-2}\right)=0 .
$$

The if-part then follows from Lemmas 3.4-3.6. For the only-if part, let $\varphi$ be a power index on $\mathscr{E}_{S}$ satisfying the Transfer Property, Symmetry, and Monotonicity.

For every $(m, w) \in M \times W$, let $P \in \mathscr{P}$ such that $(m, w) \in T(P)$ and define the number $\omega^{m, w} \in \mathbb{R}$ by $\omega^{m, w}=\varphi_{m}\left(E^{P^{m, w}}\right)$. Note that this definition is independent of the exact choice of $P$, as long as $(m, w) \in T(P)$. By symmetry of $\varphi$, we obtain $\omega^{m, w}=\varphi_{w}\left(E^{P^{m, w}}\right)$. Again by symmetry of $\varphi$, we then have $\varphi_{\ell}\left(E^{P^{m, w}}\right)=-\frac{2 \omega^{m, w}}{2 k-2}$ for every $\ell \in N \backslash\{m, w\}$. Also by symmetry of $\varphi$, we have $\varphi_{m}\left(E^{\emptyset}\right)=0$, and therefore, by Monotonicity of $\varphi, \omega^{m, w} \geq 0$. Clearly, $\Phi^{\omega}\left(E^{P^{m, w}}\right)=\varphi\left(E^{P^{m, w}}\right)$ for every $(m, w) \in M \times W$, so that $\Phi^{\omega}=\varphi$ by Lemma 
3.7. Since, in particular, $\Phi_{m}^{\omega}\left(E^{P^{m, w}}\right)=\omega^{m, w}=\varphi_{m}\left(E^{P^{m, w}}\right)$ for every $(m, w) \in$ $M \times W$, the weights are unique.

\section{Appendix B Independence of the axioms in Theorem} 3.2

The axioms in Theorem 3.2 are independent, as the following examples show. Verifications of (2) and (3) are left to the readers.

Example 3.7. (1) For every $P \in \mathscr{P}, m \in M$, and $w \in W$ let $\alpha_{m}(P)=\mid\left\{w^{\prime} \in\right.$ $\left.W:\left(m, w^{\prime}\right) \in T(P)\right\} \mid$ and $\alpha_{w}(P)=\left|\left\{m^{\prime} \in M:\left(m^{\prime}, w\right) \in T(P)\right\}\right|$. Let

$$
\beta_{\ell}(P)= \begin{cases}\frac{\alpha_{\ell}(P)}{\sum_{m \in M} \alpha_{m}(P)+\sum_{w \in W} \alpha_{w}(P)}-\frac{1}{n} & \text { if } \sum_{m \in M} \alpha_{m}(P)+\sum_{w \in W} \alpha_{w}(P) \neq 0, \\ 0 & \text { otherwise. }\end{cases}
$$

Fix an arbitrary weight system $\omega$, and define the power index $\varphi^{1}$ by

$$
\varphi^{1}\left(E^{P}\right)= \begin{cases}\beta(P) & \text { if } \sum_{m \in M} \alpha_{m}(P)+\sum_{w \in W} \alpha_{w}(P) \geq 2 \alpha_{\ell}(P) \text { for all } \ell \in N \\ \Phi^{\omega}\left(E^{P}\right) & \text { otherwise. }\end{cases}
$$

We prove that $\varphi^{1}$ is a power index satisfying Symmetry and Monotonicity, but not the Transfer Property.

Suppose that $\ell, \ell^{\prime} \in N$ are symmetric players in $E^{P}$. Then $\alpha_{\ell}(P)=\alpha_{\ell^{\prime}}(P)$, so that $\varphi_{\ell}^{1}\left(E^{P}\right)=\varphi_{\ell^{\prime}}^{1}\left(E^{P}\right)$.

Let $P, P^{\prime} \in \mathscr{P}$ and $\ell_{1}, \ell_{2} \in N$ such that $\ell_{1} \in U\left(P, \ell_{2}\right), U\left(P^{\prime}, \ell\right)=U(P, \ell)$ for all $\ell \neq \ell_{2}$, and $U\left(P^{\prime}, \ell_{2}\right)=U\left(P, \ell_{2}\right) \backslash\left\{\ell_{1}\right\}$. Suppose that $\ell_{1} \in M$ and $\ell_{2} \in W$. Clearly, $\left(\ell_{1}, \ell_{2}\right) \notin T\left(P^{\prime}\right)$. If $\left(\ell_{1}, \ell_{2}\right) \notin T(P)$, it yields that $\varphi_{\ell_{1}}^{1}\left(E^{P}\right)=\varphi_{\ell_{1}}^{1}\left(E^{P^{\prime}}\right)$. If $\left(\ell_{1}, \ell_{2}\right) \in T(P)$, then $\sum_{m \in M} \alpha_{m}\left(P^{\prime}\right)+\sum_{w \in W} \alpha_{w}\left(P^{\prime}\right)=\sum_{m \in M} \alpha_{m}(P)+$ $\sum_{w \in W} \alpha_{w}(P)-2$ and $\alpha_{\ell_{1}}\left(P^{\prime}\right)=\alpha_{\ell_{1}}(P)-1$. Thus, if $\left(\ell_{1}, \ell_{2}\right) \in T(P)$ and $\sum_{m \in M} \alpha_{m}(P)+\sum_{w \in W} \alpha_{w}(P) \geq 2 \alpha_{\ell}(P)$ for all $\ell \in N$, we have

$$
\begin{aligned}
\varphi_{\ell_{1}}^{1}\left(E^{P}\right) & =\frac{\alpha_{\ell_{1}}(P)}{\sum_{m \in M} \alpha_{m}(P)+\sum_{w \in W} \alpha_{w}(P)}-\frac{1}{n} \\
& \geq \frac{\alpha_{\ell_{1}}(P)-1}{\sum_{m \in M} \alpha_{m}(P)+\sum_{w \in W} \alpha_{w}(P)-2}-\frac{1}{n}
\end{aligned}
$$




$$
\begin{aligned}
& =\frac{\alpha_{\ell_{1}}\left(P^{\prime}\right)}{\sum_{m \in M} \alpha_{m}\left(P^{\prime}\right)+\sum_{w \in W} \alpha_{w}\left(P^{\prime}\right)}-\frac{1}{n} \\
& =\varphi_{\ell_{1}}^{1}\left(E^{P^{\prime}}\right) .
\end{aligned}
$$

Otherwise, by Lemma 3.6 we have $\varphi_{\ell_{1}}^{1}\left(E^{P}\right) \geq \varphi_{\ell_{1}}^{1}\left(E^{P^{\prime}}\right)$. As for the Transfer Property, consider Example 3.1. We have $\varphi_{m_{1}}^{1}\left(E^{P}\right)+\varphi_{m_{1}}^{1}\left(E^{Q}\right) \neq \varphi_{m_{1}}^{1}\left(E^{R}\right)+$ $\varphi_{m_{1}}^{1}\left(E^{R^{\prime}}\right)$.

(2) Let $\beta=\left(\beta_{i}\right)_{i \in N}$ be a vector of distinct real numbers with $\sum_{i \in N} \beta_{i}=0$. For every $P \in \mathscr{P}$ define $\varphi^{2}\left(E^{P}\right)=\beta$. Then $\varphi^{2}$ is a power index satisfying the Transfer Property and Monotonicity, but not Symmetry.

(3) Let $P \in \mathscr{P}$. Let $\varphi^{3}\left(E^{P}\right)=\Phi^{\omega}\left(E^{P}\right)$ where $\omega^{m, w}<0$ for all $m \in M, w \in$ $W$ and $\Phi^{\omega}$ is defined similarly as for nonnegative weights. Then $\varphi^{3}$ is a power index satisfying the Transfer Property and Symmetry, but not Monotonicity. $\triangleleft$

\section{Appendix C Proofs of Section 3.4}

The proof of Theorem 3.3 is based on a number of lemmas. In the next three lemmas, $\tau$ is a weight system.

Lemma 3.8. $\Psi^{\tau}$ satisfies the Transfer Property and Symmetry.

The proof of Lemma 3.8 is analogous to those of Lemmas 3.4 and 3.5, and therefore omitted.

Lemma 3.9. $\Psi^{\tau}$ satisfies the Dummy Property.

Proof. Let $E \in \mathscr{E}_{*}$, let $\ell \in N$ be a dummy in $E$, and let $\ell^{\prime} \in N \backslash\{\ell\}$. If $\ell^{\prime}$ is also a dummy in $E$, then by Symmetry $\Psi_{\ell}^{\tau}(E)=\Psi_{\ell^{\prime}}^{\tau}(E)$. Now suppose that $\ell^{\prime}$ is not a dummy in $E$, and suppose that $\ell, \ell^{\prime} \in M$. Then

$$
\begin{aligned}
\Psi_{\ell}^{\tau}(E) & =-\sum_{\substack{m^{\prime} \neq \ell, w, \mu: \\
\left(m^{\prime}, w\right) \in E(\mu)}} \frac{2 \tau^{m^{\prime}, w}}{n-2}-\sum_{\substack{m^{\prime} \neq \ell, \mu: \\
\left(m^{\prime}, s\right) \in E(\mu)}} \frac{\tau^{m^{\prime}}}{n-1}-\sum_{\substack{w, \mu: \\
(s, w) \in E(\mu)}} \frac{\tau^{w}}{n-1} \\
& \leq \sum_{\substack{w, \mu: \\
\left(\ell^{\prime}, w\right) \in E(\mu)}} \tau^{\ell^{\prime}, w}+\sum_{\mu:\left(\ell^{\prime}, s\right) \in E(\mu)} \tau^{\ell^{\prime}}-\sum_{\substack{m^{\prime} \neq \ell^{\prime}, w, \mu: \\
\left(m^{\prime}, w\right) \in E(\mu)}} \frac{2 \tau^{m^{\prime}, w}}{n-2}
\end{aligned}
$$




$$
\begin{aligned}
& -\sum_{\substack{m^{\prime} \neq \ell^{\prime}, \mu: \\
\left(m^{\prime}, s\right) \in E(\mu)}} \frac{\tau^{m^{\prime}}}{n-1}-\sum_{\substack{w, \mu: \\
(s, w) \in E(\mu)}} \frac{\tau^{w}}{n-1} \\
= & \Psi_{\ell^{\prime}}^{\tau}(E) .
\end{aligned}
$$

The cases $\ell, \ell^{\prime} \in W ; \ell \in M, \ell^{\prime} \in W$; and $\ell \in W, \ell^{\prime} \in M$ are similar.

Lemma 3.10. $\Psi^{\tau}$ satisfies Matching Neutrality.

Proof. Let $E, E^{\prime} \in \mathscr{E}_{*}$ such that there exists a permutation $\pi: \mathscr{M} \rightarrow \mathscr{M}$ with $E(\mu)=E^{\prime}(\pi \mu)$ for all $\mu \in \mathscr{M}$. For every $m \in M$ and $w \in W$, we have

$$
\begin{gathered}
|\{\mu:(m, w) \in E(\mu)\}|=\left|\left\{\mu:(m, w) \in E^{\prime}(\mu)\right\}\right| \\
|\{\mu:(m, s) \in E(\mu)\}|=\left|\left\{\mu:(m, s) \in E^{\prime}(\mu)\right\}\right| \\
|\{\mu:(s, w) \in E(\mu)\}|=\left|\left\{\mu:(s, w) \in E^{\prime}(\mu)\right\}\right|,
\end{gathered}
$$

from which it follows that in the expressions for $\Psi_{\ell}^{\tau}(E)$ and $\Psi_{\ell}^{\tau}\left(E^{\prime}\right)$ all terms are equal, so that $\Psi_{\ell}^{\tau}(E)=\Psi_{\ell}^{\tau}\left(E^{\prime}\right)$ for all $\ell \in N$.

We next prove that if a power index $\varphi$ on $\mathscr{E}_{*}$ satisfies the Transfer Property, then for every $E \in \mathscr{E}_{*}, \varphi(E)$ is completely determined by the values $\varphi\left(E^{m, w, \mu}\right)$ for every pair $(m, w) \in M \times W$ and every $\mu \in \mathscr{M}$, by $\varphi\left(E^{m, \mu}\right)$ for every $m \in M$ and every $\mu \in \mathscr{M}$, and by $\varphi\left(E^{w, \mu}\right)$ for every $w \in W$ and every $\mu \in \mathscr{M}$, and by $\varphi\left(E^{\emptyset}\right)$.

Lemma 3.11. Let $E \in \mathscr{E}_{*}$. Let $\varphi$ and $\psi$ be power indices on $\mathscr{E}_{*}$ satisfying the Transfer Property, and such that $\varphi\left(E^{m, w, \mu}\right)=\psi\left(E^{m, w, \mu}\right)$ for every pair $(m, w) \in$ $M \times W$ and every $\mu \in \mathscr{M}, \varphi\left(E^{m, \mu}\right)=\psi\left(E^{m, \mu}\right)$ for every $m \in M$ and every $\mu \in \mathscr{M}$, and $\varphi\left(E^{w, \mu}\right)=\psi\left(E^{w, \mu}\right)$ for every $w \in W$ and every $\mu \in \mathscr{M}$, and $\varphi\left(E^{\emptyset}\right)=\psi\left(E^{\emptyset}\right)$. Then $\varphi(E)=\psi(E)$.

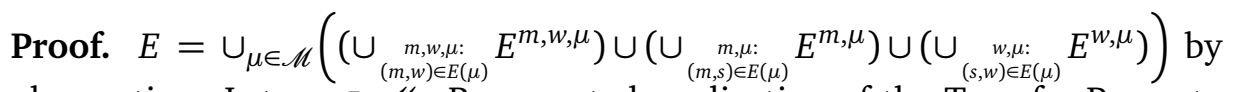
observation. Let $\mu_{1} \in \mathscr{M}$. By repeated application of the Transfer Property we have

$$
\varphi(E)=\varphi\left(\underset{\mu \in \mathscr{M} \backslash \mu_{1}}{\cup}\left(\left(\underset{\substack{m, w, \mu: \\(m, w) \in E(\mu)}}{\cup} E^{m, w, \mu}\right) \cup\left(\underset{\substack{m, \mu: \\(m, s) \in E(\mu)}}{\cup} E^{m, \mu}\right) \cup\left(\underset{\substack{w, \mu: \\(s, w) \in E(\mu)}}{\cup} E^{w, \mu}\right)\right)\right)
$$




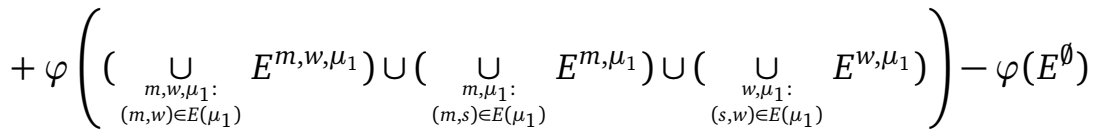

$$
\begin{aligned}
& \vdots
\end{aligned}
$$

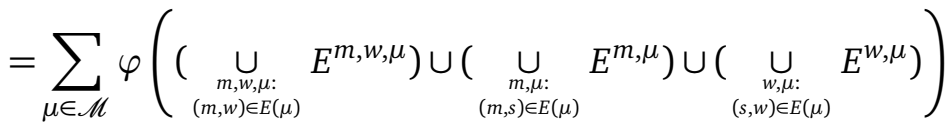

$$
\begin{aligned}
& -(|\mathscr{M}|-1) \varphi\left(E^{\emptyset}\right) \\
& \begin{aligned}
= & \sum_{\mu \in \mathscr{M}}\left(\sum_{\substack{m, w, \mu: \\
(m, w) \in E(\mu)}} \varphi\left(E^{m, w, \mu}\right)+\sum_{\substack{m, \mu: \\
(m, s) \in E(\mu)}} \varphi\left(E^{m, \mu}\right)+\sum_{\substack{w, \mu: \\
(s, w) \in E(\mu)}} \varphi\left(E^{w, \mu}\right)\right) \\
& -\left(\sum_{\mu \in \mathscr{M}}|E(\mu)|-1\right) \varphi\left(E^{\emptyset}\right) \\
= & \sum_{\mu \in \mathscr{M}}\left(\sum_{\substack{m, w, \mu: \\
(m, w) \in E(\mu)}} \psi\left(E^{m, w, \mu}\right)+\sum_{\substack{m, \mu: \\
(m, s) \in E(\mu)}} \psi\left(E^{m, \mu}\right)+\sum_{\substack{w, \mu: \\
(s, w) \in E(\mu)}} \psi\left(E^{w, \mu}\right)\right) \\
& -\left(\sum_{\mu \in \mathscr{M}}|E(\mu)|-1\right) \psi\left(E^{\emptyset}\right)
\end{aligned} \\
& \vdots
\end{aligned}
$$

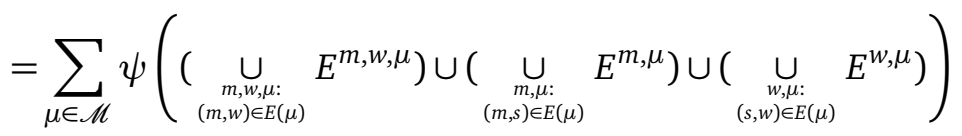

$$
\begin{aligned}
& -(|\mathscr{M}|-1) \psi\left(E^{\emptyset}\right) \\
& \vdots
\end{aligned}
$$

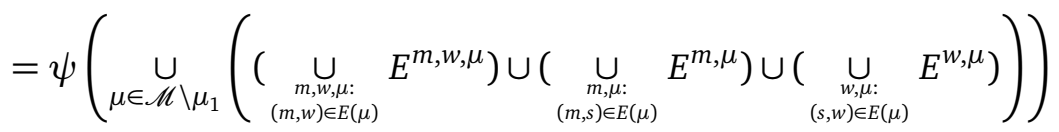

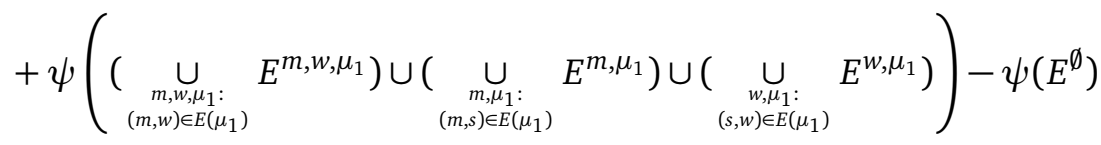

$$
\begin{aligned}
& =\psi(E) \text {, }
\end{aligned}
$$


which completes the proof of the lemma.

Now we can complete the proof of Theorem 3.3.

Proof of Theorem 3.3. Let $\tau$ be a weight system. We first prove that $\Psi^{\tau}$ is a power index. Let $E \in \mathscr{E}_{*}$. Then

$$
\begin{aligned}
\sum_{m \in M} \Psi_{m}^{\tau}(E)+\sum_{w \in W} \Psi_{w}^{\tau}(E)= & \sum_{m, w, \mu:(m, w) \in E(\mu)}\left(2 \tau^{m, w}-2(k-1) \frac{2 \tau^{m, w}}{2 k-2}\right) \\
& +\sum_{m, \mu:(m, s) \in E(\mu)}\left(\tau^{m}-(k-1) \frac{\tau^{m}}{2 k-1}-k \frac{\tau^{m}}{2 k-1}\right) \\
& +\sum_{w, \mu:(s, w) \in E(\mu)}\left(\tau^{w}-(k-1) \frac{\tau^{w}}{2 k-1}-k \frac{\tau^{w}}{2 k-1}\right) \\
= & 0 .
\end{aligned}
$$

The if-part follows from Lemmas 3.8-3.10. For the only-if-part, let $\varphi$ be a power index on $\mathscr{E}_{*}$ satisfying the Transfer Property, Symmetry, the Dummy Property, and Matching Neutrality.

For every pair $(m, w) \in M \times W$ and every $\mu \in \mathscr{M}$ such that $\mu(m)=w$, $E^{m, w, \mu}\left(\mu^{\prime}\right)=\emptyset$ for all $\mu^{\prime} \in \mathscr{M}$. By Symmetry, $\varphi_{\ell}\left(E^{m, w, \mu}\right)=\Psi_{\ell}^{\tau}\left(E^{m, w, \mu}\right)=0$ for all $\ell \in N$. For every $m \in M$ and every $\mu \in \mathscr{M}$ such that $\mu(m)=s$, $E^{m, \mu}\left(\mu^{\prime}\right)=\emptyset$ for all $\mu^{\prime} \in \mathscr{M}$. By Symmetry, $\varphi_{\ell}\left(E^{m, \mu}\right)=\Psi_{\ell}^{\tau}\left(E^{m, \mu}\right)=0$ for all $\ell \in N$. For every $w \in W$ and every $\mu \in \mathscr{M}$ such that $\mu(w)=s, E^{w, \mu}\left(\mu^{\prime}\right)=\emptyset$ for all $\mu^{\prime} \in \mathscr{M}$. By Symmetry, $\varphi_{\ell}\left(E^{w, \mu}\right)=\Psi_{\ell}^{\tau}\left(E^{w, \mu}\right)=0$ for all $\ell \in N$.

For every pair $(m, w) \in M \times W$ and matching $\mu \in \mathscr{M}$ such that $\mu(m) \neq w$, define $\tau^{m, w}=\varphi_{m}\left(E^{m, w, \mu}\right)$. For every $m \in M$ and matching $\mu \in \mathscr{M}$ such that $\mu(m) \neq s$, define $\tau^{m}=\varphi_{m}\left(E^{m, \mu}\right)$. For every $w \in W$ and matching $\mu \in \mathscr{M}$ such that $\mu(w) \neq s$, define $\tau^{w}=\varphi_{w}\left(E^{w, \mu}\right)$. By Matching Neutrality, these definitions are independent of the exact choice of $\mu$. By Symmetry of $\varphi$, we have $\varphi_{w}\left(E^{m, w, \mu}\right)=\tau^{m, w}$ and $\varphi_{\ell}\left(E^{m, w, \mu}\right)=-\frac{2 \tau^{m, w}}{n-2}$ for every dummy $\ell \in N \backslash\{m, w\}$, and $\varphi_{\ell}\left(E^{m, \mu}\right)=-\frac{\tau^{m}}{n-1}$ for every dummy $\ell \in N \backslash\{m\}$, and $\varphi_{\ell}\left(E^{w, \mu}\right)=-\frac{\tau^{w}}{n-1}$ for every dummy $\ell \in N \backslash\{w\}$, and $\varphi_{\ell}\left(E^{\emptyset}\right)=0$ for all $\ell \in N$. Therefore, by the Dummy Property of $\varphi, \tau^{m, w}, \tau^{m}, \tau^{w} \geq 0$. Moreover, $\Psi^{\tau}\left(E^{m, w, \mu}\right)=\varphi\left(E^{m, w, \mu}\right)$ and $\Psi^{\tau}\left(E^{m, \mu}\right)=\varphi\left(E^{m, \mu}\right)$ and $\Psi^{\tau}\left(E^{w, \mu}\right)=\varphi\left(E^{w, \mu}\right)$ 
and $\Psi^{\tau}\left(E^{\emptyset}\right)=\varphi\left(E^{\emptyset}\right)$, so that $\Psi^{\tau}(E)=\varphi(E)$ by Lemma 3.11. Since, in particular, $\Psi_{m}^{\tau}\left(E^{m, w, \mu}\right)=\tau^{m, w}=\varphi_{m}\left(E^{m, w, \mu}\right)$ and $\Psi_{m}^{\tau}\left(E^{m, \mu}\right)=\tau^{m}=\varphi_{m}\left(E^{m, \mu}\right)$ and $\Psi_{w}^{\tau}\left(E^{w, \mu}\right)=\tau^{w}=\varphi_{w}\left(E^{w, \mu}\right)$ for every $(m, w) \in M \times W$, every $m \in M$, and every $w \in W$, the weights are unique.

\section{Appendix D Independence of the axioms in Theorem 3.3}

The axioms in Theorem 3.3 are independent, as the following examples show. Verifications are left to the reader.

Example 3.8. (1) For every $E \in \mathscr{E}_{*}$ and every $m \in M$ and every $w \in W$, let $\alpha_{m}(E)=\left|\left\{w^{\prime} \in W, \mu \in \mathscr{M}:\left(m, w^{\prime}\right) \in E(\mu)\right\}\right|+|\{\mu \in \mathscr{M}:(m, s) \in E(\mu)\}|$ and let $\alpha_{w}(E)=\left|\left\{m^{\prime} \in M, \mu \in \mathscr{M}:\left(m^{\prime}, w\right) \in E(\mu)\right\}\right|+|\{\mu \in \mathscr{M}:(s, w) \in E(\mu)\}|$. Define

$$
\varphi_{\ell}^{4}(E)= \begin{cases}\frac{\alpha_{\ell}(E)}{\sum_{m \in M} \alpha_{m}(E)+\sum_{w \in W} \alpha_{w}(E)}-\frac{1}{n} & \text { if } \sum_{m \in M} \alpha_{m}(E)+\sum_{w \in W} \alpha_{w}(E) \neq 0, \\ 0 & \text { otherwise, }\end{cases}
$$

for every $\ell \in N$. Then $\varphi^{4}$ is a power index satisfying Symmetry, the Dummy Property and Matching Neutrality, but not the Transfer Property.

(2) For every $E \in \mathscr{E}_{*}$, let $\varphi^{5}(E)=\Psi^{\tau}(E)$ where $\tau^{m, w}, \tau^{m, s}, \tau^{s, w}<0$ for all $m \in M, w \in W$, and $\Psi^{\tau}$ is defined similarly as for nonnegative weights. Then $\varphi^{5}$ is a power index satisfying the Transfer Property, Symmetry, and Matching Neutrality, but not the Dummy Property.

(3) Let $\beta=\left(\beta_{i}\right)_{i \in N}$ be a vector of positive and distinct real numbers. Let $\tau$ be a weight system. Define

$$
\begin{aligned}
\varphi_{m}^{6}(E)= & \sum_{\substack{w, \mu: \\
(m, w) \in E(\mu)}} \beta_{m} \tau^{m, w}+\sum_{\mu:(m, s) \in E(\mu)} \beta_{m} \tau^{m}-\sum_{\substack{m^{\prime} \neq m, w, \mu: \\
\left(m^{\prime}, w\right) \in E(\mu)}} \frac{\beta_{m}\left(\beta_{m^{\prime}}+\beta_{w}\right) \tau^{m^{\prime}, w}}{\sum_{\ell \in N \backslash\left\{m^{\prime}, w\right\}} \beta_{\ell}} \\
& -\sum_{\substack{m^{\prime} \neq m, \mu: \\
\left(m^{\prime}, s\right) \in E(\mu)}} \frac{\beta_{m} \beta_{m^{\prime}} \tau^{m^{\prime}}}{\sum_{\ell \in N \backslash\left\{m^{\prime}\right\}} \beta_{\ell}}-\sum_{\substack{w, \mu: \\
(s, w) \in E(\mu)}} \frac{\beta_{m} \beta_{w} \tau^{w}}{\sum_{\ell \in N \backslash\{w\}} \beta_{\ell}}
\end{aligned}
$$




$$
\begin{aligned}
\varphi_{w}^{6}(E)= & \sum_{\substack{m, \mu: \\
(m, w) \in E(\mu)}} \beta_{w} \tau^{m, w}+\sum_{\mu:(s, w) \in E(\mu)} \beta_{w} \tau^{w}-\sum_{\substack{m, w^{\prime} \neq w, \mu: \\
\left(m, w^{\prime} \in \in E(\mu)\right.}} \frac{\beta_{w}\left(\beta_{m}+\beta_{w}^{\prime}\right) \tau^{m, w^{\prime}}}{\sum_{\ell \in N \backslash\left\{m, w^{\prime}\right\}} \beta_{\ell}} \\
& -\sum_{\substack{w, \neq w, \mu: \\
\left(s, w^{\prime}\right) \in E(\mu)}} \frac{\beta_{w} \beta_{w^{\prime}} \tau^{w^{\prime}}}{\sum_{\ell \in N \backslash\left\{w^{\prime}\right\}} \beta_{\ell}}-\sum_{\substack{m, \mu: \\
(m, s) \in E(\mu)}} \frac{\beta_{w} \beta_{m} \tau^{m}}{\sum_{\ell \in N \backslash\{m\}} \beta_{\ell}}
\end{aligned}
$$

for every $E \in \mathscr{E}_{*}$ and every $m \in M$ and every $w \in W$. Then $\varphi^{6}$ is a power index satisfying the Transfer Property, the Dummy Property, Matching Neutrality, but not Symmetry.

(4) Let $\sigma=\left(\sigma^{\mu}\right)_{\mu \in \mathscr{M}}$ be a vector of positive and distinct real numbers, and let $\tau$ be a weight system. For every $E \in \mathscr{E}_{*}, m \in M$, and $w \in W$ define

$$
\begin{aligned}
\varphi_{m}^{7}(E)= & \sum_{\substack{w, \mu: \\
(m, w) \in E(\mu)}} \sigma^{\mu} \tau^{m, w}+\sum_{\mu:(m, s) \in E(\mu)} \sigma^{\mu} \tau^{m}-\sum_{\substack{m^{\prime} \neq m, w, \mu: \\
\left(m^{\prime}, w\right) \in E(\mu)}} \frac{2 \sigma^{\mu} \tau^{m^{\prime}, w}}{n-2} \\
& -\sum_{\substack{m^{\prime} \neq m, \mu: \\
\left(m^{\prime}, s\right) \in E(\mu)}} \frac{\sigma^{\mu} \tau^{m^{\prime}}}{n-1}-\sum_{\substack{w, \mu: \\
(s, w) \in E(\mu)}} \frac{\sigma^{\mu} \tau^{w}}{n-1}, \\
\varphi_{w}^{7}(E)= & \sum_{\substack{m, \mu: \\
(m, w) \in E(\mu)}} \sigma^{\mu} \tau^{m, w}+\sum_{\mu:(s, w) \in E(\mu)} \sigma^{\mu} \tau^{w}-\sum_{\substack{m, w^{\prime} \neq w, \mu: \\
\left(m, w^{\prime}\right) \in E(\mu)}} \frac{2 \sigma^{\mu} \tau^{m, w^{\prime}}}{n-2} \\
& -\sum_{\substack{w^{\prime} \neq w, \mu: \\
\left(s, w^{\prime}\right) \in E(\mu)}} \frac{\sigma^{\mu} \tau^{w^{\prime}}}{n-1}-\sum_{\substack{m, \mu: \\
(m, s) E E(\mu)}} \frac{\sigma^{\mu} \tau^{m}}{n-1} .
\end{aligned}
$$

Then $\varphi^{7}$ is a power index satisfying the Transfer Property, Symmetry, and the Dummy Property, but not Matching Neutrality. 


\section{Chapter 4}

\section{Sequential claim games}

\subsection{Introduction}

Consider an estate and a number of players who have entitlements (rights) on this estate. In general, the sum of these entitlements is larger than the size of the estate, so that the division of the estate becomes a problem. In the literature, this problem is also known as the bankruptcy problem. It was first formally studied in O'Neill [48] and Aumann and Maschler [3]. Most of the approaches in the literature, including these seminal papers, are non-strategic or axiomatic, or both: division rules and their properties are studied, as well as cooperative games based on the bankruptcy or estate division problem. For a relatively recent survey of this literature see Thomson [66].

O'Neill [48] also proposes a strategic, noncooperative approach to the bankruptcy problem. In this approach, players simultaneously and independently put claims on parts of the estate, and each part is divided proportionally among those players who put a claim on it. This approach was thoroughly studied in Atlamaz et al. [2] for proportional sharing of claimed parts, and in Peters et al. [52] for other sharing rules from the literature on the bankruptcy problem. It was extended to include multiple estates by Pálvölgyi et al. [51].

In this chapter we also take a noncooperative approach, but now consider

Adapted from: 'Kong Q. and Peters H.: Sequential claim games. Working paper, 2020'. 
a dynamic game in which the players make their claims consecutively, in a given order. More precisely, the estate is identified with the interval $[0,1]$, each player $j$ has an entitlement $c_{j}$ with $0<c_{j}<1$, and claims a number of (disjoint) closed subintervals of $[0,1]$ with total length $c_{j}$. Player 1 starts, next it is player 2's turn, etc, until the last player $n$. This gives rise to a division of the interval $[0,1]$ into subintervals, and on each subinterval a number of claims - a so-called claims profile. Each subinterval is then divided proportionally among the claimants. Of course, the order in which the players put claims on the interval is arbitrary, and the analysis for other orders is analogous. One could also start with a chance move determining the order this would again not change the analysis.

For this so-called sequential claim game, we study strategy profiles that are a Nash equilibrium or, stronger, a subgame perfect equilibrium. We focus on myopic play: this means that a player first claims the hitherto least claimed parts, next the second least claimed parts, and so on and so forth, until the player's entitlement is exhausted. As is easy to see, such a strategy is optimal for the last player $n$, but not per se for the other players. Indeed, we show that myopic strategies do not necessarily form a Nash equilibrium, and that there can be Nash equilibria that are not myopic.

One way to make sure that myopic play is a Nash equilibrium, is to restrict the possible strategies of the players, as follows. Whenever a player puts a claim on a part of the interval on which the total claims of the preceding players are equal, then that claim is executed from left to right, without 'holes'. In other words, an action of player $j$ consists of distributing $j$ 's entitlement $c_{j}$ over subintervals with different total claims of $j$ 's predecessors; $j$ does not have a say about where the resulting claims are put exactly, instead, they are put from left to right on each subinterval. Under this restriction on the set of admissible strategies, myopic play is indeed always a Nash equilibrium: this is the first main result of this chapter, Theorem 4.6.

Myopic play, however, is not always a subgame perfect equilibrium, neither with nor without the left-right restriction on strategies. It is easy to see that for two players, in the game without (but also with) the left-right restriction on strategies, a strategy profile is a subgame perfect equilibrium if and only if the players play myopically (Proposition 4.1). This is still true for 
three players in the restricted game (Proposition 4.2), but not in the unrestricted game. For four players and in the restricted game, Proposition 4.3 gives a complete characterization of all vectors of entitlements for which myopic play is a subgame perfect equilibrium. This is quite technical, and for this reason we refrain from analyzing games with more than four players in this respect.

Instead, we then focus on the unrestricted game and consider the question how myopic play with claims put from left to right, can be turned into a subgame perfect equilibrium. We introduce the possibility of punishment: whenever, in some subgame starting with a player $j$, a preceding player has deviated, then $j$ and all following players punish the last deviating player $r$ by claiming as much as possible the intervals also claimed by $r$, as long as this is consistent with myopic play. The second main result in this chapter is that the associated strategies form a subgame perfect equilibrium (Theorem 4.7).

Just as simultaneous claim games, as in O'Neill [48] and Atlamaz et al. [2], are related to Blotto games [8], sequential claim games can be seen as sequential Blotto games. These have been and are studied in the literature, but usually as a series of simultaneous Blotto games or more generally, contests. See, e.g., Klumpp and Konrad [39] and the references therein.

Section 4.2 is devoted to definitions and a few preliminary results, Section 4.3 to myopic play as a Nash equilibrium, and Section 4.4 mainly to myopic play with punishment as a subgame perfect equilibrium. Section 4.5 contains a few remarks on equilibrium payoffs in comparison with the static approach in Atlamaz et al. [2], and Section 4.6 concludes. Some of the results and proofs are deferred to appendices.

\subsection{Model and preliminaries}

There are $n \geq 2$ players, and an estate of size one is to be distributed among these players. The size of the estate is inessential: all our results can easily be adapted for estates of any size. Players care only about their shares of the estate. Each player $j=1, \ldots, n$ has an entitlement $c_{i} \in \mathbb{R}$ with $0<c_{j}<$ 
1. Throughout, unless stated otherwise, our definitions and results are with respect to the vector of entitlements $c=\left(c_{1}, \ldots, c_{n}\right)$.

The estate is represented by the interval from zero to one, and distributed by means of a sequential claim game: there is a fixed order according to which the players claim parts of the interval, where player $j$ can claim $c_{j}$ in total. This leads to a partition of the interval, and each element of this partition is divided equally among those players who put a claim on it. This partition plus the claims will be called a 'claims profile'.

In this section we formally define claims profiles, strategies, payoffs, best replies, and Nash equilibrium. We also introduce the concept of a myopic strategy, which plays a central role in this chapter.

\subsubsection{Actions and claims profiles}

A claim is a closed subinterval of the interval $[0,1]$. An action of player $j$ is a finite number of claims with disjoint interiors and total length equal to $c_{j}$. Throughout this chapter, unless stated otherwise, we assume that claims are made consecutively by the players, in the order $1, \ldots, n$. We write $[j]=$ $\{1, \ldots, j\}$ for every $j \in\{1, \ldots, n\}$.

For any player $j$, a claims profile for $[j]$ is a triple $(y, \beta, m)$ where (i) $m \in \mathbb{N}$, (ii) $y=\left(y_{0}, \ldots, y_{m}\right) \in \mathbb{R}^{m+1}$ with $0=y_{0}<y_{1}<\ldots<y_{m-1}<y_{m}=1$, and (iii) $\beta=\left(\beta_{i}\right)_{i \in[j]}$, where $\beta_{i}:\{1, \ldots, m\} \rightarrow\{0,1\}$ such that

$$
\sum_{t=1}^{m} \beta_{i}(t)\left(y_{t}-y_{t-1}\right)=c_{i}
$$

for every $i \in[j]$. We use the notation $\beta_{[j]}(t)$ to denote the total claim on the interval $t$ between $y_{t-1}$ and $y_{t}$, i.e., $\beta_{[j]}(t)=\sum_{i=1}^{j} \beta_{i}(t)$. Thus, a claims profile for $[j]$ tells us which parts of the interval $[0,1]$ have been claimed by whom of the players $1, \ldots, j$. A claims profile for $[j]$ can be obtained by considering the coarsest common refinement of the claims of the players in $[j]$.

For a claims profile $(y, \beta, m)$ for $[j]$ and a player $h<j$, the triple $\left(y,\left(\beta_{i}\right)_{i \in[h]}\right.$, $m)$ is a claims profile for $[h]$. 


\subsubsection{Strategies and payoffs}

Consider any player $j$. A strategy $\sigma_{j}$ of player $j$ is a collection of actions of player $j$, exactly one at each claims profile for $[j-1] .{ }^{1}$ The set of all strategies of player $j$ is denoted by $\mathscr{S}_{j}$.

A strategy profile is an $n$-tuple of strategies $\sigma=\left(\sigma_{1}, \ldots, \sigma_{n}\right) \in \mathscr{S}=$ $\mathscr{S}_{1} \times \ldots \times \mathscr{I}_{n}$. A strategy profile $\sigma$ results in a sequence of claims profiles for $[1], \ldots,[n]$.

A strategy profile $\sigma$, resulting in a claims profile $(y, \beta, m)$ for $[n]$, leads to the payoff $u_{j}(\sigma)$, defined by

$$
u_{j}(\sigma)=\sum_{t \in\{1, \ldots m\}: \beta_{j}(t)=1} \frac{\beta_{j}(t)}{\beta_{[n]}(t)}\left(y_{t}-y_{t-1}\right)
$$

for every player $j \in[n]$. In words, every part of the final partition of the interval $[0,1]$ is divided equally among those players who have claimed it.

We also sometimes write $u_{j}(y, \beta, m)$ for player $j$ 's payoff from a strategy profile $\sigma$ resulting in the claims profile $(y, \beta, m)$ for $[n]$, hence

$$
u_{j}(y, \beta, m)=\sum_{t \in\{1, \ldots m\}: \beta_{j}(t)=1} \frac{\beta_{j}(t)}{\beta_{[n]}(t)}\left(y_{t}-y_{t-1}\right) .
$$

\subsubsection{Myopic strategies}

In view of our definition of the player payoffs, an obvious action for a player $j$ would be to start with claiming the (thus far) least claimed parts of the interval $[0,1]$, then the second least claimed parts, and so on, until the total entitlement $c_{j}$ is exhausted. Such an action, of course, does not take into account the possible later actions of the players $k$ with $k>j$.

Formally, at some claims profile for $[j-1]$, an action of player $j$ is myopic if it results in a claims profile $(\bar{y}, \bar{\beta}, \bar{m})$ for $[j]$ satisfying the following condition: for all $t, t^{\prime} \in\{1, \ldots, \bar{m}\}$ with $\bar{\beta}_{[j-1]}(t)<\bar{\beta}_{[j-1]}\left(t^{\prime}\right)$ and $\bar{\beta}_{j}\left(t^{\prime}\right)=1$, we have $\bar{\beta}_{j}(t)=1$. A strategy of player $j$ is myopic if all its actions are myopic. That a

\footnotetext{
${ }^{1}$ The claims profile for $[0]$ is simply $\left(y, \beta_{0}, 1\right)$ with $\beta_{0}(1)=0$, thus, the interval $[0,1]$ without any claims.
} 
player's strategy is myopic means that this player always plays optimally as if he were the last player to move in the game; this is quite intuitive and follows formally from Lemma 2.3 below.

Myopic actions and strategies play a central role in this chapter. They provide obvious candidates for Nash and subgame perfect equilibria, to be introduced later, but, as we will see, in most cases this requires additional conditions.

\subsubsection{Best replies}

Given a strategy profile $\sigma \in \mathscr{S}$, strategy $\sigma_{j} \in \mathscr{S}_{j}$ of player $j$ is a best reply against the strategy profile $\sigma_{-j}=\left(\sigma_{1}, \ldots, \sigma_{j-1}, \sigma_{j+1}, \ldots, \sigma_{n}\right)$ of the other players if for every strategy $\sigma_{j}^{\prime} \in \mathscr{S}_{j}$ of player $j$, we have

$$
u_{j}(\sigma) \geq u_{j}\left(\sigma_{-j}, \sigma_{j}^{\prime}\right)
$$

As already announced, for the last player myopic actions are optimal. This is formalized in the following lemma.

Lemma 4.1. Let $\sigma$ be a strategy profile. Then $\sigma_{n}$ is a best reply against $\sigma_{-n}$ if, and only if, the action of player $n$ at the claims profile for $[n-1]$ resulting from $\sigma$, is myopic.

Proof. Let $(y, \beta, m)$ be the claims profile for $[n]$ resulting from $\sigma$, and suppose that the action of player $n$ is not myopic at the claims profile for $[n-1]$ resulting from $\sigma$. Since player $n$ 's action is not myopic, there are $t, t^{\prime} \in$ $\{1, \ldots, m\}$ such that $\beta_{[n-1]}(t)<\beta_{[n-1]}\left(t^{\prime}\right), \beta_{n}\left(t^{\prime}\right)=1$, and $\beta_{n}(t)=0$. Let $\varepsilon=\min \left\{y_{t}-y_{t-1}, y_{t^{\prime}}-y_{t^{\prime}-1}\right\}$. A change of player $n^{\prime}$ s action by including the claim $\left[y_{t-1}, y_{t-1}+\varepsilon\right]$ and excluding the claim $\left[y_{t^{\prime}-1}, y_{t^{\prime}-1}+\varepsilon\right]$, results in a payoff loss of $\varepsilon /\left(\beta_{[n-1]}\left(t^{\prime}\right)+1\right)$ and a payoff gain of $\varepsilon /\left(\beta_{[n-1]}(t)+1\right)$. Since $\beta_{[n-1]}(t)<\beta_{[n-1]}\left(t^{\prime}\right)$, this implies that player $n$ has a net payoff gain. This completes the proof of the only-if direction of the lemma.

For the if-direction, consider any action of player $n$ at the claims profile for $[n-1]$ that is not myopic. By applying a finite number of changes as above, each time increasing player $n$ 's payoff, we obtain a myopic action in 
the end. Moreover, every myopic action of player $n$ results in the same payoff for that player. This completes the if-direction of the lemma.

\subsubsection{Myopic strategies and Nash equilibrium}

A strategy profile $\sigma \in \mathscr{S}$ in which every player plays a best reply, is a Nash equilibrium.

We first show, by an example, that a myopic strategy profile does not have to be a Nash equilibrium.

The left-right myopic action (or strategy) of player 1 is simply to claim $\left[0, c_{1}\right]$. The left-right myopic action of player $j>1$ at some claims profile for $[j-1]$ is the myopic action such that in the resulting claims profile $(y, \beta, m)$ for $[j]$ we have: for all $t, t^{\prime} \in\{1, \ldots, m\}$ such that $t^{\prime}>t$ and $\beta_{[j-1]}\left(t^{\prime}\right)=$ $\beta_{[j-1]}(t)$, if $\beta_{j}\left(t^{\prime}\right)=1$ then $\beta_{j}(t)=1$. In words, player $j$ plays myopically such that, on every level of the claims profile for $[j-1]$, player $j$ 's claims are put from left to right without 'holes'.

The strategy of player $j$ in which all actions are left-right myopic, is called player j's left-right myopic strategy, and is denoted by $\mu_{j}$. The left-right myopic strategy profile is denoted by $\mu$, i.e., $\mu=\left(\mu_{1}, \ldots, \mu_{n}\right)$.

The following two examples show that myopic strategy profiles, including left-right myopic strategy profiles, may fail to be Nash equilibria.

Example 4.1. (Cf. Figure 4.1.) Let $n=3, c_{1}=c_{3}=\frac{4}{8}$, and $c_{2}=\frac{3}{8}$. Let $\sigma=\left(\mu_{1}, \mu_{2}, \sigma_{3}\right)$, where $\sigma_{3}$ is defined as follows: at the claims profile for [2] where player 1 claims $\left[0, \frac{4}{8}\right]$ and player 2 claims $\left[\frac{4}{8}, \frac{7}{8}\right]$, player 3 claims $\left[\frac{4}{8}, 1\right]$; in all other cases $\sigma_{3}$ prescribes the left-right myopic action. Then $\sigma$ results in the claims profile for [3] in which player 1 claims $\left[0, \frac{4}{8}\right]$, players 2 claims $\left[\frac{4}{8}, \frac{7}{8}\right]$, and player 3 claims $\left[\frac{4}{8}, 1\right]$, resulting in payoff $\frac{3}{16}$ for player 2. If player 2 , at the claims profile for [1] at which player 1 claims $\left[0, \frac{4}{8}\right]$, instead claims $\left[0, \frac{2}{8}\right] \cup\left[\frac{4}{8}, \frac{5}{8}\right]$, then this results in the claims profile for [3] in which player 1 claims $\left[0, \frac{4}{8}\right]$, players 2 claims $\left[0, \frac{2}{8}\right] \cup\left[\frac{4}{8}, \frac{5}{8}\right]$, and player 3 claims $\left[\frac{2}{8}, \frac{3}{8}\right] \cup\left[\frac{5}{8}, 1\right]$, resulting in payoff $\frac{4}{16}$ for player 2 . Hence $\sigma$ is not a Nash equilibrium although all actions are myopic. 


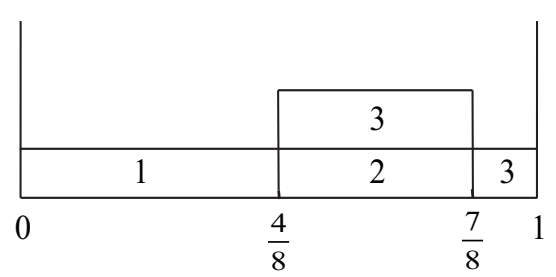

(a)

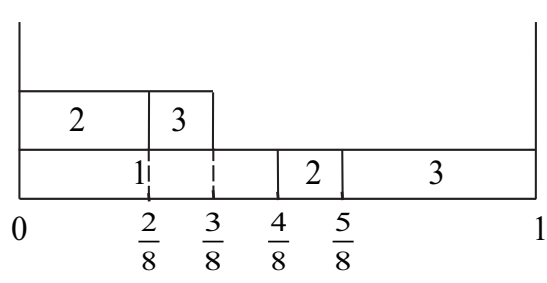

(b)

FIGURE 4.1: Example 4.1. Part (a) corresponds to $\sigma$ and part

(b) corresponds to the case when player 2 deviates to claim

$$
\left[0, \frac{2}{8}\right] \cup\left[\frac{4}{8}, \frac{5}{8}\right]
$$

The next example shows that also the left-right myopic strategy profile does not have to be a Nash equilibrium.

Example 4.2. (Cf. Figure 4.2.) As in the preceding example, let $n=3, c_{1}=$ $c_{3}=\frac{4}{8}$, and $c_{2}=\frac{3}{8}$. Now consider the strategy profile $\mu=\left(\mu_{1}, \mu_{2}, \mu_{3}\right)$. By Lemma 4.1, $\mu_{3}$ is a best reply against $\left(\mu_{1}, \mu_{2}\right)$. Also, it is not hard to see that $\mu_{2}$ is a best reply against $\left(\mu_{1}, \mu_{3}\right)$ : player 2 obtains a payoff of $\frac{3}{8}$, which is equal to player 2's entitlement and therefore maximal. By playing $\mu_{1}$ against $\left(\mu_{2}, \mu_{3}\right)$, player 1 obtains a payoff of $\frac{5}{16}$, whereas claiming $\left[\frac{4}{8}, 1\right]$ yields $\frac{8}{16}$. Hence $\mu$ is not a Nash equilibrium.

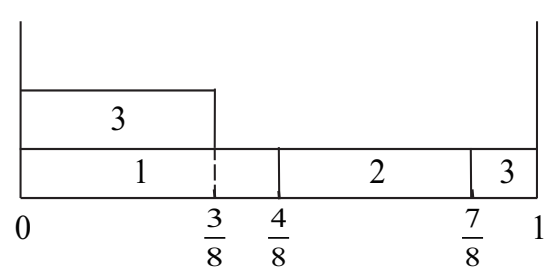

(a)

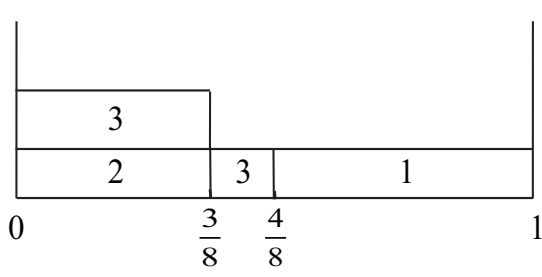

(b)

FIgURE 4.2: Example 4.2. Part (a) corresponds to $\mu$ and part (b) corresponds to the case when player 1 deviates to claim $\left[\frac{4}{8}, 1\right]$

Intuitively, that (for instance) a myopic strategy profile, and in particular the left-right myopic strategy profile, fails to be a Nash equilibrium is caused by the fact that players are completely free where to deposit their claims. Therefore, in the next section, we will put a restriction on this. 
We conclude the present section by showing that, if a player acts myopically, then the difference between the most claimed part and the least claimed part of the interval, if positive, can only decrease. For any player $j \in[n]$ and claims profile $(y, \beta, m)$ for $[j]$, we introduce the notation

$$
\delta(y, \beta, m)=\max \left\{\beta_{[j]}(t)-\beta_{[j]}\left(t^{\prime}\right): t, t^{\prime} \in\{1, \ldots, m\}\right\}
$$

for this difference.

Lemma 4.2. Let $\sigma$ be a strategy profile, let $j \in[n-1]$, let $(y, \beta, m)$ be the claims profile for $[j]$ and let $\left(y^{\prime}, \beta^{\prime}, m^{\prime}\right)$ be the claims profile for $[j+1]$, resulting from $\sigma$. Suppose $j+1$ 's action at $(y, \beta, m)$ according to $\sigma_{j+1}$, is myopic. Then (i) if $\delta(y, \beta, m)=0$, then $\delta\left(y^{\prime}, \beta^{\prime}, m^{\prime}\right)=1$, and (ii) if $\delta(y, \beta, m) \geq 1$, then $\delta\left(y^{\prime}, \beta^{\prime}, m^{\prime}\right) \leq \delta(y, \beta, m)$.

Proof. (i) If $\delta(y, \beta, m)=0$, then for any action of player $j+1$, since $0<$ $c_{j+1}<1$, we have $\delta\left(y^{\prime}, \beta^{\prime}, m^{\prime}\right)=1$. (ii) Now let $\delta(y, \beta, m) \geq 1$. Without loss of generality we may assume that $(y, \beta, m)$ is the claims profile for $[j]$ induced by $\left(y^{\prime}, \beta^{\prime}, m^{\prime}\right)$, i.e., $(y, \beta, m)=\left(y^{\prime}, \beta_{i \in[j]}^{\prime}, m^{\prime}\right)$. Consider any $s, t \in$ $\left\{1, \ldots, m^{\prime}\right\}$ with $\beta_{[j]}^{\prime}(s)>\beta_{[j]}^{\prime}(t)$. Since player $j+1$ 's action is myopic, this implies that if $\beta_{[j+1]}^{\prime}(s)=\beta_{[j]}^{\prime}(s)+1$, then also $\beta_{[j+1]}^{\prime}(t)=\beta_{[j]}^{\prime}(t)+1$. Hence, $\delta\left(y^{\prime}, \beta^{\prime}, m^{\prime}\right) \leq \delta(y, \beta, m)$.

\subsection{Left-right restricted claims}

In this section we put a restriction on where players can deposit their claims. Specifically, we assume that each player's claims on parts of the interval on which the total claims of the predecessors are equal, have to be positioned from left to right without 'holes'. In other words, a player has the freedom to choose claims in terms of the levels of the claims profile for that player's predecessors, but not where to put these claims exactly.

Formally, this means that the unique strategy of player 1 is to claim $\left[0, c_{1}\right]$ - in fact, this is $\mu_{1}$. For player $j>1$ and any claims profile $(y, \beta, m)$ for $[j-1]$ this means that an action of player $j$ results in a claims profile $(\bar{y}, \bar{\beta}, \bar{m})$ for [j] such that for all $t, t^{\prime} \in\{1, \ldots, \bar{m}\}$ with $t^{\prime}>t$ and $\bar{\beta}_{[j-1]}\left(t^{\prime}\right)=\bar{\beta}_{[j-1]}(t)$, if 
$\beta_{j}\left(t^{\prime}\right)=1$ then $\beta_{j}(t)=1$. This says that, if player $j$ claims two parts of the interval $[0,1]$ on which the total claims of his predecessors are equal, then player $j$ should start with claiming the left most part.

For each $j$ we denote the resulting collection of strategies by $\widetilde{\mathscr{S}_{j}}$, and we write $\widetilde{\mathscr{S}}=\widetilde{\mathscr{S}_{1}} \times \ldots \times \widetilde{\mathscr{S}}_{n}$. Best reply and Nash equilibrium are defined as before, but now restricted to $\widetilde{\mathscr{S}}$.

Clearly, for every $j \in[n]$, acting myopically is equivalent to acting leftright myopically in $\widetilde{\mathscr{S}_{j}}$; and the left-right myopic strategy-profile $\mu$ is in $\widetilde{\mathscr{S}}$.

Our main result in this section (Theorem 4.6) will be that $\mu$ is a Nash equilibrium in $\widetilde{\mathscr{S}}$. To prove this result we need three lemmas, the proofs of which can be found in Appendix A.

A claims profile $(y, \beta, m)$ for $[j]$ is decreasing if $\beta_{[j]}(t) \geq \beta_{[j]}\left(t^{\prime}\right)$ for all $t, t^{\prime} \in\{1, \ldots, m\}$ with $t<t^{\prime}$. Intuitively, a strategy profile in $\widetilde{\mathscr{S}}$ results in a decreasing claims profile, and this is indeed what the first lemma says.

Lemma 4.3. Let $\in \widetilde{\mathscr{S}}$ be a strategy profile, let $j \in[n]$, and let $(y, \beta, m)$ be the resulting claims profile for $[j]$. Then $(y, \beta, m)$ is decreasing.

The second lemma says that, if the difference between the most and least claimed parts in the claims profile for [ $j]$ is equal to 1 , player $j+1$ does not act myopically but all players after $j+1$ play their myopic strategies, and the difference between the most and least claimed parts in the claims profile for $[n]$ is at least 2 , then player $j+1$ obtains a strictly higher payoff by acting myopically.

Lemma 4.4. Let $j \in[n-2]$ and let $\sigma \in \widetilde{\mathscr{S}}$ be a strategy profile such that $\sigma_{k}=\mu_{k}$ for all $k \in[n]$ with $k>j+1$. Let $(y, \beta, m)$ and $(\bar{y}, \bar{\beta}, \bar{m})$ be the claims profiles for $[j]$ and $[n]$, respectively, resulting from $\sigma$. Let $\delta(y, \beta, m)=1$, and let $\delta(\bar{y}, \bar{\beta}, \bar{m}) \geq 2$. Then

(a) player $j+1$ does not act myopically at $(y, \beta, m)$,

(b) $\delta(\bar{y}, \bar{\beta}, \bar{m})=2$, and

(c) if $\sigma_{j+1}^{\prime} \in \widetilde{\mathscr{S}_{j+1}}$ is equal to $\sigma_{j+1}$ except that $j+1$ acts myopically at $(y, \beta, m)$, then $u_{j+1}\left(\sigma_{-(j+1)}, \sigma_{j+1}^{\prime}\right)>u_{j+1}(\sigma)$. 
The third lemma needed to prove Theorem 4.6, considers a similar situation as in Lemma 4.4, but now with final maximal difference at most 1 . The lemma shows that then, switching to the myopic action, cannot decrease the payoff.

Lemma 4.5. Let $j \in[n-2]$ and let $\sigma \in \widetilde{\mathscr{S}}$ be a strategy profile such that $\sigma_{k}=\mu_{k}$ for all $k \in[n]$ with $k>j+1$. Let $(y, \beta, m)$ and $(\bar{y}, \bar{\beta}, \bar{m})$ be the claims profiles for $[j]$ and $[n]$, respectively, resulting from $\sigma$. Let $\delta(y, \beta, m)=1$, and let $\delta(\bar{y}, \bar{\beta}, \bar{m}) \leq 1$. Suppose player $j+1$ does not act myopically at $(y, \beta, m)$. If $\sigma_{j+1}^{\prime} \in \widetilde{\mathscr{S}}_{j+1}$ is equal to $\sigma_{j+1}$ except that $j+1$ acts myopically at $(y, \beta, m)$, then $u_{j+1}\left(\sigma_{-(j+1)}, \sigma_{j+1}^{\prime}\right) \geq u_{j+1}(\sigma)$.

We are now sufficiently equipped to prove the announced result.

Theorem 4.6. $\mu$ is a Nash equilibrium in $\widetilde{\mathscr{S}}$.

Proof. Since player 1's strategy is by definition fixed at $\mu_{1}$, and since, by Lemma 4.1, $\mu_{n}$ is a best reply of player $n$ against any strategy profile $\sigma_{-n}$, it is sufficient to show that for player $j+1$ with $1<j<n-1, \mu_{j+1}$ is a best reply against $\mu_{-(j+1)}$. Let $(y, \beta, m)$ be the claims profile for $[j]$ resulting from $\mu$. Then $\delta(y, \beta, m) \leq 1$ since all players in [j] act myopically. If $\delta(y, \beta, m)=0$, then player $j+1$ acts myopically by definition. Now assume that $\delta(y, \beta, m)=$ 1. If $j+1$ does not act myopically and for the resulting final claims profile $(\bar{y}, \bar{\beta}, \bar{m})$ for $[n]$ we have $\delta(\bar{y}, \bar{\beta}, \bar{m}) \geq 2$, then by Lemma 4.4 player $j+1$ has a strictly higher payoff by acting myopically. If $\delta(\bar{y}, \bar{\beta}, \bar{m}) \leq 1$, then by Lemma 4.5 player $j+1$ has a payoff at least as high by acting myopically. This completes the proof.

As the following examples show, there may be Nash equilibria (quite) different from $\mu$. In the first of these examples, all five players except player 3 play their myopic strategies. Player 3 does not act myopically on the equilibrium path.

Example 4.3. (Cf. Figure 4.3.) Let $n=5, c_{1}=c_{2}=c_{5}=\frac{2}{3}, c_{3}=\frac{5}{12}$, and $c_{4}=\frac{1}{3}$. Consider the strategy profile $\sigma \in \widetilde{\mathscr{S}}$ in which all players except player 3 play myopically, i.e., $\sigma_{i}=\mu_{i}$ for $i=1,2,4,5$ (note that $\sigma_{1}=\mu_{1}$ by definition). As to $\sigma_{3}$, at the claims profile for [2] where 2 has claimed [0, $\frac{1}{3}$ ] 
and $\left[\frac{2}{3}, 1\right]$, hence player 2 acts myopically (1 has claimed $\left[0, \frac{2}{3}\right]$ ), let player 3 claim $\left[0, \frac{1}{12}\right]$ and $\left[\frac{1}{3}, \frac{2}{3}\right]$; in all other cases, let player 3 act myopically. This is a Nash equilibrium, as we will briefly show below. On the equilibrium path of this equilibrium, player 3 does not act myopically. Of course, by Theorem 4.6, the payoff to player 3 does not change if player 3 plays the myopic strategy: indeed, in both cases the payoff is $\frac{5}{36}$; and in fact, the payoffs for all players stay the same in this case.

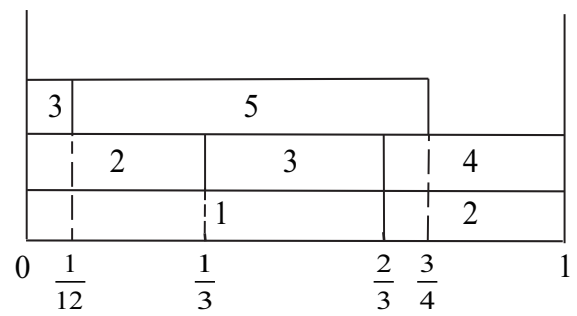

(a)

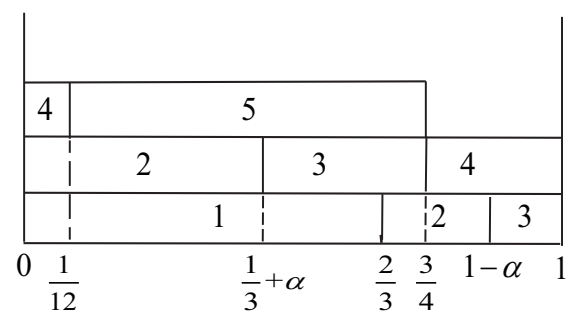

(b)

FIGURE 4.3: Example 4.3. Part (a) corresponds to $\sigma$ and part (b) to the case when player 2 deviates.

To check that $\sigma$ is a Nash equilibrium, we start by considering player 2 . If, instead of acting myopically, player 2 claims $\left[0, \frac{1}{3}+\alpha\right]$ and $\left[\frac{2}{3}, 1-\alpha\right]$ for some $0<\alpha \leq \frac{1}{3}$, then players 3 , 4, and 5 will act myopically and player 2 's payoff will be $\frac{19}{72}-\frac{\alpha}{6}$ for $0<\alpha \leq \frac{1}{4}$, and $\frac{16}{72}$ for $\frac{1}{4}<\alpha \leq \frac{1}{3}$; these payoffs are strictly smaller than the payoff $\frac{19}{72}$ from acting myopically. For player 3 , any other action will result in the same payoff $\frac{5}{36}$. For player 4 any other claim than $\left[\frac{2}{3}, 1\right]$ results in a strictly lower payoff, and also player 5 strictly looses from not playing myopically.

The next example exhibits a Nash equilibrium where no player except player 1 always acts myopically. On the equilibrium path, player 3 does not act myopically. The payoffs, however, are still the same as in the myopic equilibrium.

Example 4.4. (Cf. Figure 4.4.) While the strategy profile in Example 4.3 is still close to myopic play, we now exhibit an example where no player $j>1$ plays $\mu_{j}$. Let $n=4, c_{1}=\frac{1}{2}, c_{2}=\frac{1}{6}$, and $c_{3}=c_{4}=\frac{2}{3}$. We define the strategy profile $\sigma \in \widetilde{\mathscr{S}}$ as follows. Player 1 , of course, claims [0, $\left.\frac{1}{2}\right]$. Player 2 claims 
$\left[0, \frac{1}{6}\right]$. If player 2 claims $\left[0, \frac{1}{6}\right]$, then player 3 acts myopically; otherwise, if player 2 claims $[0, \alpha]$ and $\left[\frac{1}{2}, \frac{2}{3}-\alpha\right]$ for some $0 \leq \alpha<\frac{1}{6}$, then player 3 claims $\left[\alpha, \frac{2}{3}+\alpha\right]$. Finally, if player 2 claims $\left[0, \frac{1}{6}\right]$, then player 4 acts myopically; otherwise, player 4 claims $\left[0, \frac{2}{3}\right]$. It is not difficult to verify that no player can gain from deviating, so $\sigma$ is a Nash equilibrium. As in Example 2.1, the payoffs from $\sigma$ are the same as those from $\mu$.

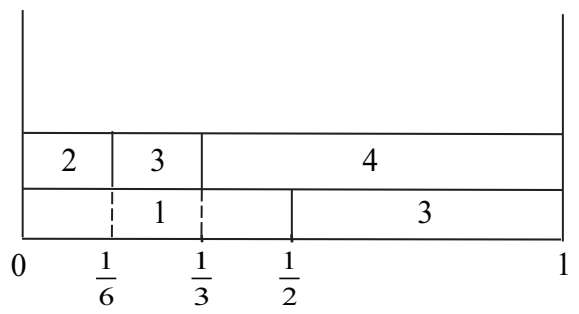

(a)

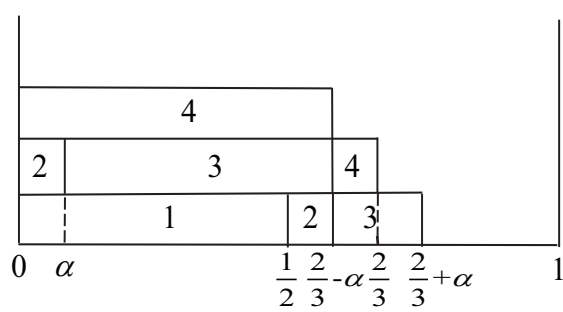

(b)

FigURE 4.4: Example 2.4. Part (a) corresponds to $\sigma$ and part

(b) to the case when player 2 deviates to $0 \leq \alpha<\frac{1}{6}$.

The following example exhibits a Nash equilibrium with payoffs different from the myopic strategy profile.

Example 4.5. Let $n=3, c_{1}=\frac{3}{8}, c_{2}=\frac{2}{8}$, and $c_{3}=\frac{6}{8}$. We define a strategy profile $\sigma \in \widetilde{\mathscr{S}}$, as follows. Player 1 , of course, claims [0, $\left.\frac{3}{8}\right]$. Player 2's (nonmyopic) strategy is to claim $\left[0, \frac{1}{8}\right]$ and $\left[\frac{3}{8}, \frac{4}{8}\right]$. Player 3's strategy is as follows. Suppose player 2 claims $\left[0, \frac{2}{8}-\alpha\right]$ and $\left[\frac{3}{8}, \frac{3}{8}+\alpha\right]$, where $0 \leq \alpha \leq \frac{2}{8}$. If $0 \leq \alpha \leq \frac{1}{8}$, then player 3 acts myopically; otherwise, if $\frac{1}{8}<\alpha \leq \frac{2}{8}$, then player 3 claims $\left[\frac{2}{8}-\alpha, 1-\alpha\right]$. The payoffs from this strategy-profile $\sigma$ are $\left(\frac{3}{16}, \frac{3}{16}, \frac{10}{16}\right)$. We claim that $\sigma$ is a Nash equilibrium. If $\alpha=\frac{1}{8}$, then player 3 acts myopically, which is a best reply. If $\alpha<\frac{1}{8}$, then player 2 obtains $\frac{1}{8}+\frac{1}{2} \alpha$, which is smaller than $\frac{3}{16}$; if $\alpha>\frac{1}{8}$, then player 2 obtains $\frac{1}{8}$, which is again smaller than $\frac{3}{16}$. The Nash equilibrium $\mu$ results in the payoffs $\left(\frac{3}{16}, \frac{4}{16}, \frac{9}{16}\right)$, which is better for player 2 (who does not act myopically in $\sigma$ ), but worse for player 3 (who acts myopically in $\sigma$ ).

The final example extends the previous one. Now, there are two players who do not act myopically on the equilibrium path. 
Example 4.6. We add a player 4 to the game in Example 4.5, with $c_{4}=\frac{5}{8}$. We now define a strategy profile $\sigma \in \widetilde{\mathscr{S}}$ as follows. Player 1 claims $\left[0, \frac{3}{8}\right]$ and player 2 claims $\left[0, \frac{1}{8}\right]$ and $\left[\frac{3}{8}, \frac{4}{8}\right]$, as in the preceding example. Suppose player 2 claims $\left[0, \frac{2}{8}-\alpha\right]$ and $\left[\frac{3}{8}, \frac{3}{8}+\alpha\right]$, where $0 \leq \alpha \leq \frac{2}{8}$. If $0 \leq \alpha \leq \frac{1}{8}$, then player 3 claims $\left[0, \frac{1}{8}+\alpha\right]$ and $\left[\frac{3}{8}+\alpha, 1\right]$; otherwise, if $\frac{1}{8}<\alpha \leq \frac{2}{8}$, then player 3 claims $\left[0, \frac{6}{8}\right]$. Player 4 , finally, acts myopically if $0 \leq \alpha \leq \frac{1}{8}$; otherwise, if $\frac{1}{8}<\alpha \leq \frac{2}{8}$, player 4 claims $\left[0, \frac{5}{8}\right]$. The payoffs from this strategy profile $\sigma$ are $\left(\frac{8}{48}, \frac{5}{48}, \frac{20}{48}, \frac{15}{48}\right)$. It can again be verified that $\sigma$ is a Nash equilibrium. On the equilibrium path, both player 2 and player 3 do not act myopically. If the resulting final claims profile (for [4]) is $(y, \beta, m)$, then $\beta_{[4]}(1)=3$ and $\beta_{[4]}(m)=1$, so that $\delta(y, \beta, m)=2$. The payoffs from $\mu$ are $\left(\frac{9}{48}, \frac{6}{48}, \frac{18}{48}, \frac{15}{48}\right)$ : in particular, player 3 receives a higher payoff in $\sigma$, where player 3 does not act myopically, than in $\mu$.

From these examples we may draw a few conclusions. There can be Nash equilibria in $\widetilde{\mathscr{S}}$ which are different from $\mu$. In such a Nash equilibrium it may happen that the maximal difference between the numbers of claims on different parts of the interval exceeds one. Also, a player in a Nash equilibrium who does not act myopically on the equilibrium path, may have a higher payoff than in the myopic Nash equilibrium.

In general, it seems practically infeasible to find all Nash equilibria in $\widetilde{\mathscr{S}}$ : even for the case of three players this is an extensive task, as we show in Appendix B. We do not expect this to be easier if we drop the left-right restriction on claims, i.e., in $\mathscr{S}$.

Clearly, some of the equilibria above exhibit non-optimal play off the equilibrium path. In the next section, we strengthen the condition of Nash equilibrium to subgame perfect equilibrium.

\subsection{Subgame perfect equilibrium}

Let $\sigma \in \mathscr{S}$ be a strategy profile, let $j \in[n-1]$, and let $(y, \beta, m)$ be a claims profile for $[j]$. We identify $(y, \beta, m)$ with a subgame for $\{j+1, \ldots, n\}$ in the obvious way; and $\sigma$ induces a strategy profile $\left(\sigma_{j+1}, \ldots, \sigma_{n}\right)$ in this subgame. 
A strategy profile $\sigma \in \mathscr{S}$ is a subgame perfect equilibrium if for every player $j \in[n-1]$ and every claims profile $(y, \beta, m)$ for $[j]$, the strategy profile $\left(\sigma_{j+1}, \ldots, \sigma_{n}\right)$ is a Nash equilibrium in the subgame $(y, \beta, m)$.

The definition of a subgame perfect equilibrium in $\widetilde{\mathscr{S}}$ is similar, with the understanding that only subgames are considered that can arise from strategy profiles in which each player $h$ plays a strategy in $\widetilde{\mathscr{S}_{h}}$. In particular, those subgames are decreasing (Lemma 4.3).

Checking if a strategy profile $\sigma$ is a subgame perfect equilibrium can be a tedious task.

Example 4.7. It is easy to see that none of the strategy profiles $\sigma \in \widetilde{\mathscr{S}}$ in Examples 4.4-4.6 is a subgame perfect equilibrium; this follows, basically, from Lemma 4.1, since the last player (player 4 in Examples 4.4 and 4.6, and player 3 in Example 4.5) does not act myopically in every subgame.

As to $\sigma \in \widetilde{\mathscr{S}}$ in Example 4.3, since $\sigma$ is a Nash equilibrium, $\left(\sigma_{2}, \ldots, \sigma_{5}\right)$ is also a Nash equilibrium in the unique subgame for $\{2, \ldots, 5\}$. Any subgame for $\{3,4,5\}$ is a claims profile for [2], where $\left[0, \frac{2}{3}-\alpha\right]$ is claimed twice, and $\left[\frac{2}{3}-\alpha, \frac{2}{3}+\alpha\right]$ is claimed once, for some $0 \leq \alpha \leq \frac{1}{3}$. If $\alpha=\frac{1}{3}$, then this is a subgame on the equilibrium path of $\sigma$, and therefore $\left(\sigma_{3}, \sigma_{4}, \sigma_{5}\right)$ is a Nash equilibrium. Otherwise, for every action of player 3 , it can be seen that players 4 and 5 , acting myopically, will jointly claim $[0,1]$, so that for player 3 it is as if he were the last player; therefore acting myopically is a best reply for player 3 , hence, also in this case, $\left(\sigma_{3}, \sigma_{4}, \sigma_{5}\right)$ is a Nash equilibrium. In any subgame for $\{4,5\}$, it can be seen that player 4 acting myopically is a best reply, given that player 5 acts myopically, and therefore $\left(\sigma_{4}, \sigma_{5}\right)$ is a Nash equilibrium. Finally, acting myopically (hence, according to $\sigma_{5}$ ) is a best reply for player 5 in any subgame (basically, by Lemma 4.1).

By an only slight modification of this argument, it follows that in Example 4.3, also $\mu$ is a subgame perfect equilibrium in $\widetilde{\mathscr{S}}$. In Example 4.6, however, $\mu$ is not a subgame perfect equilibrium in $\widetilde{\mathscr{S}}$. Consider a subgame for $\{3,4\}$ where player 2 has claimed $\left[0, \frac{1}{8}\right]$ and $\left[\frac{3}{8}, \frac{4}{8}\right]$. If player 3 acts myopically, followed by player 4 acting myopically, then the payoff to player 3 is $\frac{9}{24}$. If, however, player 3 claims $\left[0, \frac{2}{8}\right]$ and $\left[\frac{4}{8}, 1\right]$, followed by player 4 acting myopically, then the payoff to player 3 is $\frac{10}{24}$. Thus, $\mu$ is not a subgame perfect 
equilibrium.

\subsubsection{Subgame perfect equilibrium for $n \in\{2,3,4\}$}

As the example (4.7, or 4.6) shows, $\mu$ is not necessarily a subgame perfect equilibrium, not even in $\widetilde{\mathscr{S}}$. In this example, $n=4$. For two players we have the following observation (which we state without proof).

Proposition 4.1. Let $n=2$. Then a strategy profile in $\mathscr{S}$ is a subgame perfect equilibrium if and only if it is myopic.

For three players, Example 4.2 shows that $\mu$ is not necessarily a Nash equilibrium and therefore not necessarily a subgame perfect equilibrium in $\mathscr{S}$. We do have the following result.

Proposition 4.2. Let $n=3$. Then $\sigma$ is a subgame perfect equilibrium in $\widetilde{\mathscr{S}}$ if and only if $\sigma=\mu$.

Proof. We leave it to the reader to verify that $\mu$ is a subgame perfect equilibrium in $\widetilde{\mathscr{S}}$. Conversely, let $\sigma$ be a subgame perfect equilibrium in $\widetilde{\mathscr{S}}$. Then by definition of $\widetilde{\mathscr{S}_{1}}$, we have $\sigma_{1}=\mu_{1}$. By definition of $\widetilde{\mathscr{S}_{3}}$ and Lemma 4.1 we have $\sigma_{3}=\mu_{3}$. Consider $\sigma_{2}$ and suppose, contrary to what we want to prove, that player 2 claims $[0, \alpha] \cup\left[c_{1}, c_{1}+\gamma\right]$ for $\alpha+\gamma=c_{2}$ such that $0<\alpha \leq c_{1} \leq c_{1}+\beta<1$. Let $\varepsilon>0$ be a small number, and suppose that player 2 instead claims $[0, \alpha-\varepsilon] \cup\left[c_{1}, c_{1}+\gamma+\varepsilon\right]$. If $c_{3} \leq 1-\alpha$ then this change improves player 2's payoff by $\varepsilon-\frac{\varepsilon}{2}=\frac{\varepsilon}{2}$, and if $c_{3}>1-\alpha$ then then this change improves player 2's payoff by $\frac{\varepsilon}{2}-\frac{\varepsilon}{3}=\frac{\varepsilon}{6}$. Therefore, $\sigma_{2}=\mu_{2}$.

The following result gives the necessary and sufficient conditions for $\mu$ to be a subgame perfect equilibrium in $\widetilde{\mathscr{S}}$ in case there are four players. The proof of this result is rather elaborate, and can be found in Appendix C.

Let $r=\left(c_{1}+\ldots+c_{4}\right) \bmod 1$.

Proposition 4.3. Let $n=4$.

(i) If $c_{1}+c_{2}+c_{3}+c_{4} \leq 2$, then $\mu$ is a subgame perfect equilibrium in $\widetilde{\mathscr{S}}$ if and only if

$$
\max \left\{0,1-c_{3}-c_{4}\right\} \geq \min \left\{c_{1}, c_{2}, c_{1}+c_{2}+c_{3}-1\right\} .
$$


(ii) If $c_{1}+c_{2}+c_{3}+c_{4}>2$, then $\mu$ is a subgame perfect equilibrium in $\widetilde{\mathscr{S}}$ if and only if the following three conditions hold.

(ii.1) $\max \left\{1-c_{3}, c_{1}+c_{2}+\frac{2}{3} c_{3}+c_{4}-\frac{5}{3}\right\} \geq \min \left\{c_{1}, c_{2}, 1-c_{4}, 3-c_{1}-c_{2}-3 c_{4}\right\}$.

(ii.2) $c_{1}+c_{2}+c_{4}-1 \geq \min \left\{c_{1}, c_{2}, 1-c_{4}\right\}$.

(ii.3) $\max \left\{c_{1}+c_{2}-1, \frac{3}{2} r\right\} \geq \min \left\{c_{1}, c_{2}, 1-c_{3}, 1-c_{4}, c_{1}+c_{2}+c_{3}-1-\frac{3}{2} r\right\}$.

Example 4.8. In Example 4.6, $\left(c_{1}, c_{2}, c_{3}, c_{4}\right)=\frac{1}{8}(3,2,6,5)$, hence $c_{1}+\cdots+$ $c_{4}=2$. For $\mu$ to be a subgame perfect equilibrium we would need $\max \{0,1-$ $\left.c_{3}-c_{4}\right\} \geq \min \left\{c_{1}, c_{2}, c_{1}+c_{2}+c_{3}-1\right\}$ according to Proposition 4.3(i), which is clearly not the case.

As the conditions in Proposition 4.3 are rather technical, we refrain from pursuing the case $n>4$. Instead, in the next subsection, we will identify a subgame perfect equilibrium, based on myopic play with punishment, in $\mathscr{S}$.

\subsubsection{Myopic play with punishment}

In this subsection we consider the set of strategy profiles $\mathscr{S}$. We will show that left-right myopic play can be a subgame perfect equilibrium provided we build in a way to punish deviators. In words, this works as follows. Suppose a player $j$ deviates by not acting left-right myopically. This means that player $j$ does not act myopically - claims some interval whereas intervals with lower total claims of $j$ 's predecessors are still available - or acts myopically but with claims not always from left to right, that is, leaving 'holes'. Then we will assume that all players $i>j$ play myopically, as much as possible from left to right, but with the exception that, when choosing between intervals with equal total preceding claims, they first claim - from left to right - the intervals claimed by player $j$.

Formally, let $i, j \in[n]$ with $i<j$ and consider a(ny) claims profile for $[j-1]$. The lrm-i action ('lrm' stands for left-right myopic) of player $j$ is the myopic action of $j$, resulting in the claims profile $(y, \beta, m)$ for $[j]$, such that for all $t^{\prime}, t \in\{1, \ldots, m\}$ with $t^{\prime}>t$ and $\beta_{[j-1]}\left(t^{\prime}\right)=\beta_{[j-1]}(t)$ we have:

(i) if $\beta_{i}(t)=0$ and $\beta_{i}\left(t^{\prime}\right)=1$, then $\beta_{j}(t)=1$ implies $\beta_{j}\left(t^{\prime}\right)=1$, 
(ii) in all (three) other cases, $\beta_{j}\left(t^{\prime}\right)=1$ implies $\beta_{j}(t)=1$.

Next, for any claims profile for $[j-1]$, we define the red card player $\hat{r}$ as follows. If every player $1, \ldots, j-1$ has acted left-right myopically, then $\hat{r}=0$. Otherwise, let $r_{1}$ be the first player who has not acted left-right myopically. If every player $r_{1}+1, \ldots, j-1$ has used the lrm- $r_{1}$ action, then $\hat{r}=r_{1}$. Otherwise, let $r_{2}$ be the first player after $r_{1}$ who has not used the lrm- $r_{1}$ action. If every player $r_{2}+1, \ldots, j-1$ has used the lrm- $r_{2}$ action, then $\hat{r}=r_{2}$. And so on and so forth.

By $\hat{\mu}_{j} \in \mathscr{S}_{j}$ we denote the strategy of player $j$ according to which $j$ plays the $1 \mathrm{rm}-\hat{r}$ action at each claims profile for $[j-1], \hat{r}$ being the red card player of that profile, with lrm-0 being the left-right myopic action. Let $\hat{\mu}=\left(\hat{\mu}_{1}, \ldots, \hat{\mu}_{n}\right)$.

Theorem 4.7. $\hat{\mu}$ is a subgame perfect equilibrium in $\mathscr{S}$.

The proof of this result can be found in Appendix D.

\subsection{Payoffs}

In Atlamaz et al. [2] a static version of the claim game is studied. In this static game, a strategy of a player is simply a collection of claims, and players play simultaneously and independently. Apart from the static nature, another difference with our approach is that in Atlamaz et al. [2] multiple claims are allowed: an interval can be claimed more than once by the same player. Here, we reconsider this static game, but without allowing for multiple claims, thus, following O'Neill [48]. A Nash equilibrium profile in the static game is a claims profile such that no player can gain by deviating to a different strategy. We omit the (relatively straightforward) proof of the the following theorem - it is the single-claim version of Theorem 1 in Atlamaz et al. [2]. Recall that $\delta(y, \beta, m)$ denotes the maximal difference in total claims of a claims profile $(y, \beta, m)$.

Proposition 4.4. Claims profile $(y, \beta, m)$ is a Nash equilibrium profile if and only if $\delta(y, \beta, m) \leq 1$. 
From Proposition 4.4 the possible payoffs from Nash equilibrium profiles can be characterized. Let $r=\sum_{i=1}^{n} c_{i} \bmod 1$, hence $\sum_{i=1}^{n} c_{i}=K+r$ for some $K \in \mathbb{N} \cup\{0\}$. Then, at a Nash equilibrium profile $(y, \beta, m)$ we have $\beta_{[n]}(t) \in\{K, K+1\}$ for every $t=1, \ldots, m$. For every player $j$, let $r_{j}$ be the part of $c_{j}$ invested in intervals $t$ with $\beta_{[n]}(t)=K+1$. Then $r_{j} \leq c_{j}, r_{j} \leq r$, and $c_{j}-r_{j} \leq 1-r$, hence for every $j \in[n]$ we have

$$
\sum_{i=1}^{n} r_{i}=(K+1) r, \text { and } \max \left\{0, c_{j}-(1-r)\right\} \leq r_{j} \leq \min \left\{c_{j}, r\right\} .
$$

It is not hard to show that, conversely, for every vector $\left(r_{1}, \ldots, r_{n}\right)$ satisfying (4.1) there is an associated Nash equilibrium claims profile. In particular, the set of Nash equilibrium profile payoff vectors is a convex set.

Returning to the sequential claim game, since $\hat{\mu}$ is a subgame perfect equilibrium in $\mathscr{S}$ (Theorem 4.7), it is also a Nash equilibrium (in $\mathscr{S}$ ), and the resulting claims profile is a Nash equilibrium profile, as follows from Proposition 4.4. To obtain a meaningful general comparison, however, we at least need to consider all possible orders in which the players make their claims. The next example shows that even in that case there may be payoffs associated with Nash equilibrium profiles in the static game that cannot be obtained by sequential left-right myopic play in any order, nor even by convex combinations of these.

Example 4.9. Let $n=4, c_{1}=c_{2}=\frac{3}{7}, c_{3}=\frac{4}{7}$, and $c_{4}=\frac{5}{7}$, and consider the Nash equilibrium profile of the static game given by $(y, \beta, m)$ in Figure 4.5. The payoff to player 4 in this Nash equilibrium profile is equal to $\frac{5}{14}$. In all 24 possible orders of left-right myopic play in the sequential game, however, player 4 always obtains $\frac{7}{21}$, as is straightforward to verify.

It is an open question whether payoffs from Nash equilibrium profiles of the static game can always be obtained by some Nash equilibrium of the sequential game, or as convex combinations of such payoffs, and considering all $n$ ! orders in which the players can make claims. On the other hand, there can be Nash equilibrium payoffs of the sequential game that cannot be obtained 


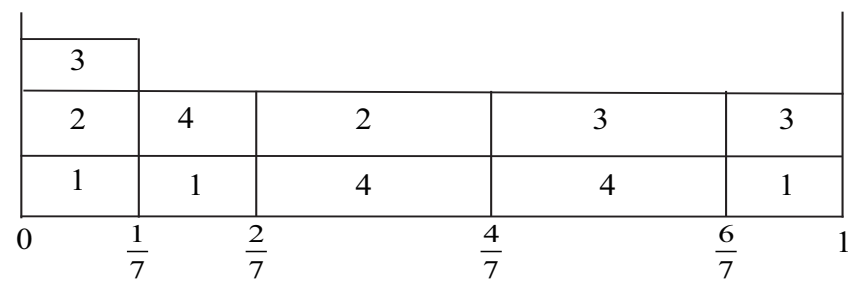

FIGURE 4.5: Example 4.9

as payoffs of Nash equilibrium profiles of the static game, as the following example shows.

Example 4.10. Consider the Nash equilibrium $\sigma$ in Example 4.6. This is a Nash equilibrium in $\widetilde{\mathscr{S}}$ but it is not difficult to reformulate it as a Nash equilibrium in $\mathscr{S}$. In this example, there is only one payoff vector that can be obtained in a Nash equilibrium of the static game, since the sum of the entitlements is equal to 2 , namely the payoff vector $\left(\frac{9}{48}, \frac{6}{48}, \frac{18}{48}, \frac{15}{48}\right)$. The payoffs from $\sigma$, however, are equal to $\left(\frac{8}{48}, \frac{5}{48}, \frac{20}{48}, \frac{15}{48}\right)$.

$\triangleleft$

\subsection{Concluding remarks}

In this chapter we studied noncooperative dynamic games, so-called sequential claim games, in order to solve the estate division problem. The main results are that myopic play is always a Nash equilibrium provided that claims on the same level are always ordered from left to right (Theorem 4.6), and that left-right myopic play with punishment is always a subgame perfect equilibrium in the general case (Theorem 4.7).

Many open questions are left. These include, of course, characterizing all Nash equilibria and subgame perfect Nash equilibria, but answering these questions may be intractable.

A conjecture is that in any subgame perfect equilibrium the maximal difference in the final claims profile is at most one - so far we did not manage to prove this. 
Finally, there are many possible extensions, including: allowing for multiple claims, considering sharing functions other than proportional sharing, and multiple estates.

\section{Appendix A Remaining proofs from Section 4.3}

Proof of Lemma 4.3. Let $t, t^{\prime} \in\{1, \ldots, m\}$ with $t<t^{\prime}$. We prove that $\beta_{[j]}(t) \geq \beta_{[j]}\left(t^{\prime}\right)$.

Without loss of generality we may take, for every player $k<j$, the claims profile equal to $\left(y,\left(\beta_{i}\right)_{i \in[k]}, m\right)$. We now consider three cases.

(i) $\beta_{[j-1]}(t)>\beta_{[j-1]}\left(t^{\prime}\right)$. In this case, since $\beta_{j}(t), \beta_{j}\left(t^{\prime}\right) \in\{0,1\}$, it follows that $\beta_{[j]}(t) \geq \beta_{[j]}\left(t^{\prime}\right)$.

(ii) $\beta_{[j-1]}(t)=\beta_{[j-1]}\left(t^{\prime}\right)$. By definition of an action we have $\beta_{j}(t)=$ $1, \beta_{j}\left(t^{\prime}\right)=0$ or $\beta_{j}(t)=1, \beta_{j}\left(t^{\prime}\right)=1$ or $\beta_{j}(t)=0, \beta_{j}\left(t^{\prime}\right)=0$. In each of these three cases, $\beta_{[j]}(t) \geq \beta_{[j]}\left(t^{\prime}\right)$.

(iii) $\beta_{[j-1]}(t)<\beta_{[j-1]}\left(t^{\prime}\right)$. Then by analogous arguments as in cases (i) and (ii) we have $\beta_{[j-2]}(t)<\beta_{[j-2]}\left(t^{\prime}\right)$. By repeating this argument we obtain $\beta_{[1]}(t)<\beta_{[1]}\left(t^{\prime}\right)$. This contradicts the definition of $\sigma_{1}\left(=\mu_{1}\right)$ and completes the proof of the lemma.

Proof of Lemma 4.4. (a) If player $j+1$ would act myopically at $(y, \beta, m)$, then by repeated application of Lemma $4.2, \delta(\bar{y}, \bar{\beta}, \bar{m}) \leq \delta(y, \beta, m)=1$. Hence, player $j+1$ does not act myopically at $(y, \beta, m)$.

(b) Since player $j+1$ does not act myopically at $(y, \beta, m)$ and $\delta(y, \beta, m)=$ 1 , we have that $\delta\left(y^{\prime}, \beta^{\prime}, m^{\prime}\right)=2$, where $\left(y^{\prime}, \beta^{\prime}, m^{\prime}\right)$ is the claims profile for $[j+1]$ resulting from $\sigma$. By repeated application of Lemma 4.2 it follows that $\delta(\bar{y}, \bar{\beta}, \bar{m}) \leq 2$. Since, by assumption, $\delta(\bar{y}, \bar{\beta}, \bar{m}) \geq 2$, we obtain $\delta(\bar{y}, \bar{\beta}, \bar{m})=$ 2.

(c) By Lemma 4.2 and parts (a) and (b), we have $\delta\left(y^{k}, \beta^{k}, m^{k}\right)=2$ for every $k>j$, where $\left(y^{k}, \beta^{k}, m^{k}\right)$ denotes the profile for $[k]$ resulting from $\sigma$, 
which is decreasing by Lemma 4.3. For every $k>j$ let

$$
\alpha_{k}=\max _{\ell \leq m^{k}}\left\{y_{\ell}^{k}: \beta_{[k]}^{k}(\ell)=\beta_{[k]}^{k}(1)\right\} \text { and } \gamma_{k}=\min _{\ell \leq m^{k}}\left\{y_{\ell-1}^{k}: \beta_{[k]}^{k}(\ell)=\beta_{[k]}^{k}\left(m^{k}\right)\right\} \text {, }
$$

i.e., $\alpha_{k}$ is the point of the interval where total claims decrease from the highest to the medium value, and $\gamma_{k}$ is the point of the interval where total claims decrease from the medium to the lowest value. (Cf. Figure 4.6.) Clearly, $0<\alpha_{k}<\gamma_{k}<1$. Using the same definition we also have $0<\alpha_{j}=\gamma_{j}<1$.

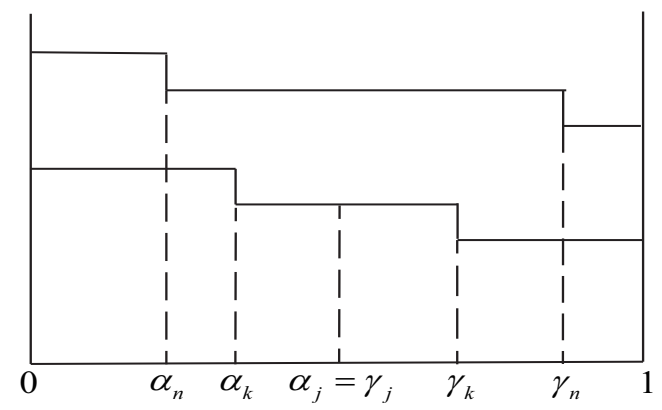

FIGURE 4.6: Proof of Lemma 4.4

Now fix a player $k$ with $j<k<n$, and consider player $k+1$. There are three cases:

(i) $c_{k+1}<1-\gamma_{k}$. Since player $k+1$ acts myopically, we obtain $\alpha_{k+1}=\alpha_{k}$ and $\gamma_{k}<\gamma_{k+1}<1$.

(ii) $1-\gamma_{k} \leq c_{k+1} \leq 1-\alpha_{k}$. Since player $k+1$ acts myopically, this would imply that $\delta\left(y^{k+1}, \beta^{k+1}, m^{k+1}\right)=1$, a contradiction. Hence this case cannot occur.

(iii) $c_{k+1}>1-\alpha_{k}$. Since player $k+1$ acts myopically, we obtain $0<\alpha_{k+1}<$ $\alpha_{k}$ and $\gamma_{k+1}=\gamma_{k}<1$.

From applying these cases for the players $j+2, \ldots, n$, consecutively, we obtain $0<\alpha_{n} \leq \alpha_{j+1}<\alpha_{j}<\gamma_{j+1} \leq \gamma_{n}<1$, where at least one of the weak inequalities is strict. We can also write $\{j+2, \ldots, n\}=L \cup H$, where $L=\{\ell$ : $\left.c_{\ell}<1-\gamma_{\ell-1}\right\}$ and $H=\left\{\ell: c_{\ell}>1-\alpha_{\ell-1}\right\}$, as a consequence of these three 
cases. Then the payoff to player $j+1$ is given by

$$
u_{j+1}(\sigma)=\frac{\alpha_{n}}{\beta_{[j]}(1)+|H|+1}+\frac{c_{j+1}-\alpha_{n}}{\beta_{[j]}(1)+|H|} .
$$

Now suppose that player $j+1$, instead, acts myopically at $(y, \beta, m)$, i.e., switches to $\sigma_{j+1}^{\prime}$, and let $(\tilde{y}, \widetilde{\beta}, \widetilde{m})$ be the claims profile for $[n]$ resulting from $\left(\sigma_{-(j+1)}, \sigma_{j+1}^{\prime}\right)$. Then, by Lemma $4.3, \delta(\widetilde{y}, \widetilde{\beta}, \widetilde{m}) \leq 1$. In this profile, the number of claims on the interval $[0, r]$, with $r=\sum_{i \in[n]} c_{i} \bmod 1$, exceeds the number of claims on the interval $[r, 1]$ by one. We distinguish two cases.

Case 1: $\alpha_{n}+\gamma_{n} \leq 1$. In this case, $r=\alpha_{n}+\gamma_{n}$, and the number of claims on $[0, r]$ is equal to $\beta_{[j]}(1)+|H|$. Player $j+1$ 's payoff is

$$
u_{j+1}\left(\sigma_{-(j+1)}, \sigma_{j+1}^{\prime}\right)=\frac{c_{j+1}-\eta}{\beta_{[j]}(1)+|H|}+\frac{\eta}{\beta_{[j]}(1)+|H|-1},
$$

where $\eta=\min \left\{1-\alpha_{n}-\gamma_{n}, \max \left\{0, c_{j+1}-\left(\alpha_{n}+\gamma_{n}-\alpha_{j}\right)\right\}\right\}$ is such that player $j+1$ claims $[r, r+\eta]$. By (4.2), $u_{j+1}\left(\sigma_{-(j+1)}, \sigma_{j+1}^{\prime}\right)>u_{j+1}(\sigma)$.

Case 2: $\alpha_{n}+\gamma_{n}>1$. In this case, $r=\alpha_{n}+\gamma_{n}-1$, and the number of claims on $[0, r]$ is equal to $\beta_{[j]}(1)+|H|+1$. Player $j+1$ 's payoff is

$$
u_{j+1}\left(\sigma_{-(j+1)}, \sigma_{j+1}^{\prime}\right)=\frac{c_{j+1}-\zeta}{\beta_{[j]}(1)+|H|}+\frac{\zeta}{\beta_{[j]}(1)+|H|+1},
$$

where $0 \leq \zeta \leq \alpha_{n}+\gamma_{n}-1$ is such that player $j+1$ claims [0, $\left.\zeta\right]$. Since $\alpha_{n}+\gamma_{n}-1<\alpha_{n}$, (4.2) implies that $u_{j+1}\left(\sigma_{-(j+1)}, \sigma_{j+1}^{\prime}\right)>u_{j+1}(\sigma)$. This concludes the proof of (c), and of the lemma.

Proof of Lemma 4.5. Let $y_{s}=\max \left\{y_{\ell}: \beta_{[j]}(\ell)=\beta_{[j]}(1)\right\}$. Since player $j+1$ does not act myopically at $(y, \beta, m)$, there is $0<x \leq y_{s}<1$ with $x \leq c_{j+1}$ and $y_{s}+c_{j+1}-x<1$, such that player $j+1$ claims the intervals $[0, x]$ and $\left[y_{s}, y_{s}+c_{j+1}-x\right]$. If player $j+1$ acts according to $\sigma_{j+1}^{\prime}$ and, thus, myopically, there is a $0 \leq z<x$ such that player $j+1$ claims the intervals $[0, z]$ (possibly of length zero, namely if $c_{j+1} \leq 1-y_{s}$ ) and $\left[y_{s}, y_{s}+c_{j+1}-z\right]$. Write $\sum_{i \in[n]} c_{i}=p+r$ for some $p \in \mathbb{N} \cup\{0\}$ and $0<r<1$. (Observe that $r$ is the point where total end claims drop by 1 , independent of whether $\sigma_{j+1}$ 
or $\sigma_{j+1}^{\prime}$ is played.) We distinguish three cases.

(i) $r \geq x$. Then switching to myopic play results in a payoff loss of $(x-z) /(p+$ 1) and a payoff gain of at least $(x-z) /(p+1)$.

(ii) $z<r<x$. Then switching to myopic play results in a payoff loss of $(r-z) /(p+1)+(x-r) / p$ and a payoff gain of $(x-z) / p$.

(iii) $0 \leq r \leq z$. Then switching to myopic play results in a payoff loss of $(x-z) / p$ and a payoff gain of $(x-z) / p$.

Hence, in each case the payoff gain is larger than or equal to the payoff loss.

\section{Appendix B Nash equilibria in $\widetilde{\mathscr{S}}$ for three players}

This section provides a complete analysis of the Nash equilibria in $\widetilde{\mathscr{S}}$ for the case of three players, $n=3$.

Player 1 always claims $\left[0, c_{1}\right]$. Suppose player 2 claims $\left[c_{1}, c_{1}+\alpha\right]$ and $\left[0, c_{2}-\alpha\right]$, where $\alpha \in\left[0, \min \left\{1-c_{1}, c_{2}\right\}\right]$. Let $f(\alpha)$ denote player 2's payoff when player 3 acts myopically. Let $g(\alpha)$ denote player 2's minimal payoff when player 3 does not act myopically. Lemmas 4.8-4.10 concern this number $g(\alpha)$.

Lemma 4.8. Let $c_{3}<c_{1}+c_{2}$. If (1) $c_{2}+c_{3} \leq c_{1}$, or (2) $c_{2}+c_{3}>c_{1}$ and $\alpha \geq c_{2}-c_{1}+c_{3}$, then $g(\alpha)$ is obtained if player 3 claims $\left[0, c_{3}\right]$. In that case

$$
g(\alpha)= \begin{cases}\frac{\alpha}{2}+\frac{c_{2}}{2}-\frac{c_{3}}{6}, & \text { if } 0 \leq \alpha \leq c_{2}-c_{3}, \\ \frac{2 \alpha}{3}+\frac{c_{2}}{3}, & \text { if } c_{2}-c_{3}<\alpha \leq \min \left\{c_{2}, 1-c_{1}\right\} .\end{cases}
$$

Proof. Both (1) and (2) imply $c_{2}-\alpha+c_{3} \leq c_{1}$. Thus, player 3 claims [0, $\left.c_{3}\right]$. The expression for $g(\alpha)$ is obtained by comparing $c_{3}$ and $c_{2}-\alpha$.

Lemma 4.9. Let $c_{3}<c_{1}+c_{2}, c_{2}+c_{3}>c_{1}$, and $\frac{c_{2}-c_{1}+c_{3}}{2} \leq \alpha<c_{2}-c_{1}+c_{3}$, i.e., $c_{1}<c_{2}-\alpha+c_{3} \leq c_{1}+\alpha$. Then:

(1) If $c_{1} \leq c_{3}$, then $g(\alpha)=\alpha+\frac{c_{1}-c_{3}}{2}$, obtained by player 3 claiming $\left[c_{2}-\right.$ $\left.\alpha, c_{2}-\alpha+c_{3}\right]$. 
(2) If $c_{3}<c_{1}$, then

$$
g(\alpha)= \begin{cases}\min \left\{\frac{\alpha}{2}+\frac{c_{2}}{2}-\frac{c_{3}}{6}, \alpha+\frac{c_{1}-c_{3}}{2}\right\} & \text { if } 0 \leq \alpha \leq c_{2}-c_{3} \\ \min \left\{\frac{2 \alpha}{3}+\frac{c_{2}}{3}, \alpha+\frac{c_{1}-c_{3}}{2}\right\} & \text { if } c_{2}-c_{3}<\alpha \leq \min \left\{c_{2}, 1-c_{1}\right\}\end{cases}
$$

is obtained by player 3 claiming $\left[0, c_{3}\right]$ or $\left[c_{2}-\alpha, c_{2}+c_{3}-\alpha\right]$.

Proof. Suppose that player 3 claims $[0, \varepsilon]$ and $\left[c_{2}-\alpha, c_{2}-\alpha+c_{3}-\varepsilon\right]$ with $\varepsilon \in\left[0, \min \left\{c_{2}-\alpha+c_{3}-c_{1}, c_{2}-\alpha\right\}\right]$. If $c_{1} \leq c_{3}$, i.e., $c_{2}-\alpha \leq c_{2}-\alpha+c_{3}-c_{1}$ (here, $c_{2}-\alpha+c_{3}-c_{1}$ is the amount that player 3 puts on $\left[c_{1}, c_{1}+\alpha\right]$ ), player 2's payoff increases with $\varepsilon \in\left[0, c_{2}-\alpha\right]$. Thus, player 3 claims $\left[c_{2}-\alpha, c_{2}-\alpha+c_{3}\right]$, and player 2's payoff is $c_{1}-c_{2}-c_{3}+2 \alpha+\frac{c_{2}-\left(c_{1}-c_{2}-c_{3}+2 \alpha\right)}{2}=\alpha+\frac{c_{1}-c_{3}}{2}$. If $c_{1}>c_{3}$, player 2's payoff increases with $\varepsilon \in\left[0, c_{2}-\alpha+c_{3}-c_{1}\right]$, and decreases with $\varepsilon \in\left[c_{2}-\alpha+c_{3}-c_{1}, c_{2}-\alpha\right]$.

Lemma 4.10. Let $c_{3}<c_{1}+c_{2}, c_{2}+c_{3}>c_{1}$, and $0 \leq \alpha<\frac{c_{2}-c_{1}+c_{3}}{2}$, i.e., $c_{1}+\alpha<c_{2}-\alpha+c_{3}$. Then:

(1) If $c_{1} \leq c_{3}$ and $\alpha \leq c_{3}-c_{1}$, then $g(\alpha)=\frac{\alpha}{6}+\frac{c_{2}}{3}$, obtained by player 3 claiming $\left[0, c_{3}\right]$.

(2) If $c_{1} \leq c_{3}$ and $\alpha>c_{3}-c_{1}$, then $g(\alpha)=\frac{\alpha}{3}+\frac{c_{1}+2 c_{2}-c_{3}}{6}$, obtained by player 3 claiming $\left[0, c_{3}-c_{1}+c_{2}-2 \alpha\right]$ and $\left[c_{2}-\alpha, c_{1}+\alpha\right]$.

(3) If $c_{3}<c_{1}$, then

$$
g(\alpha)= \begin{cases}\min \left\{\frac{\alpha}{2}+\frac{c_{2}}{2}-\frac{c_{3}}{6}, \frac{\alpha}{3}+\frac{c_{1}+2 c_{2}-c_{3}}{6}\right\} & \text { if } 0 \leq \alpha \leq c_{2}-c_{3} \\ \min \left\{\frac{2 \alpha}{3}+\frac{c_{2}}{3}, \frac{\alpha}{3}+\frac{c_{1}+2 c_{2}-c_{3}}{6}\right\} & \text { if } c_{2}-c_{3}<\alpha \leq \min \left\{c_{2}, 1-c_{1}\right\}\end{cases}
$$

is obtained by player 3 claiming $\left[0, c_{3}\right]$, or $\left[0, c_{3}-c_{1}+c_{2}-2 \alpha\right]$ and $\left[c_{2}-\alpha, c_{1}+\alpha\right]$.

The proof of Lemma 4.10 is similar to the proof of Lemma 4.9 and therefore omitted.

The following lemma is straightforward. 
Lemma 4.11. Let $c_{1}+c_{2} \leq 1$. Then

$$
f(\alpha)= \begin{cases}\frac{\alpha}{2}+\frac{c_{2}}{2}, & \text { if } c_{2}+c_{3} \leq 1 \\ \frac{\alpha}{6}+\frac{1+2 c_{2}-c_{3}}{6}, & \text { if } c_{2}+c_{3}>1 \text { and } 0 \leq \alpha \leq c_{2}+c_{3}-1 \\ \frac{\alpha}{2}+\frac{1-c_{3}}{2}, & \text { if } c_{2}+c_{3}>1 \text { and } c_{2}+c_{3}-1<\alpha \leq c_{2}\end{cases}
$$

In the next three lemmas we compute the maximal value of $g(\alpha)$.

Lemma 4.12. Let $c_{3} \geq c_{1}+c_{2}$. Then $\max _{\alpha \in\left[0, c_{2}\right]} g(\alpha)=g\left(c_{2}\right)=\frac{c_{2}}{2}$.

Proof. For $c_{3} \geq c_{1}+c_{2}, g(\alpha)$ is obtained by player 3 claiming at least $\left[0, c_{2}-\alpha\right]$ and $\left[c_{1}, c_{1}+\alpha\right]$. Then, $g(\alpha)=\frac{c_{2}-\alpha}{3}+\frac{\alpha}{2}=\frac{\alpha}{6}+\frac{c_{2}}{3}$, which implies the lemma.

Lemma 4.13. If (1) $c_{3}<c_{1}+c_{2} \leq 1$ and $c_{2}+c_{3} \leq c_{1}$ or (2) $c_{3}<c_{1}+c_{2} \leq 1$, $c_{2}+c_{3}>c_{1}$ and $c_{3}<c_{1}$, then $\max _{\alpha \in\left[0, c_{2}\right]} g(\alpha)=g\left(c_{2}\right)=c_{2}$.

Proof. If (1) holds, then the statement follows from Lemma 4.8. If (2) holds, we have $0<\frac{c_{2}-c_{1}+c_{3}}{2}<c_{2}-c_{1}+c_{3} \leq c_{2}$ and $c_{2}-c_{3}<c_{2}$. Thus, by Lemma 4.8, part (2) of Lemma 4.9, and part (3) of Lemma 4.10, the statement follows.

Lemma 4.14. Let $c_{3}<c_{1}+c_{2} \leq 1, c_{2}+c_{3}>c_{1}$ and $c_{1} \leq c_{3}$. Then $\max _{\alpha \in\left[0, c_{2}\right]} g(\alpha)$ $=g\left(c_{2}\right)=c_{2}+\frac{c_{1}-c_{3}}{2}$.

Proof. We have $0 \leq c_{3}-c_{1}<\frac{c_{2}-c_{1}+c_{3}}{2} \leq c_{2} \leq c_{2}-c_{1}+c_{3}$. The statement now follows from part (1) of Lemma 4.9 and parts (1) and (2) of Lemma 4.10.

Now we can determine all Nash equilibria for the case $0<c_{1}+c_{2} \leq 1$.

Theorem 4.15. Let $0<c_{1}+c_{2} \leq 1$.

(1) If $c_{3} \geq c_{1}+c_{2}$ and $c_{2}+c_{3} \leq 1$, then every $\alpha \in\left[0, c_{2}\right]$ results in a Nash equilibrium.

(2) If $c_{3} \geq c_{1}+c_{2}$ and $c_{2}+c_{3}>1$, then every $\alpha \in\left[c_{2}+c_{3}-1, c_{2}\right]$ results in a Nash equilibrium.

(3) If $c_{3}<c_{1}+c_{2}$ and $c_{2}+c_{3} \leq c_{1}$, then $\mu$ is the only Nash equilibrium.

(4) If $c_{3}<c_{1}+c_{2}, c_{2}+c_{3}>c_{1}$, and $c_{3}<c_{1}$, then $\mu$ is the only Nash equilibrium. 
(5) If $c_{3}<c_{1}+c_{2}, c_{1}<c_{2}+c_{3} \leq 1$, and $c_{1} \leq c_{3}$, then every $\alpha \in\left[c_{1}+c_{2}-c_{3}, c_{2}\right]$ results in a Nash equilibrium.

(6) If $c_{3}<c_{1}+c_{2}, c_{2}+c_{3}>1$, and $c_{1} \leq c_{3}$, then every $\alpha \in\left[c_{1}+2 c_{2}-1, c_{2}\right]$ results in a Nash equilibrium.

Proof. Follows from Lemmas 4.11-4.14.

We now consider the case $c_{1}+c_{2}>1$.

Lemma 4.16. Let $c_{1}+c_{2}>1>c_{3}$. Then

$f(\alpha)= \begin{cases}\frac{\alpha}{2}+\frac{c_{2}}{2}, & \text { if } c_{2}+c_{3} \leq 1, \\ \frac{\alpha}{2}+\frac{1-c_{3}}{2}, & \text { if } c_{2}+c_{3}>1 \text { and } c_{[3]} \leq 2 \text { and } c_{2}+c_{3}-1<\alpha \leq 1-c_{1}, \\ \frac{\alpha}{6}+\frac{1+2 c_{2}-c_{3}}{6}, & \text { otherwise. }\end{cases}$

Lemma 4.17. Let $c_{3}<1<c_{1}+c_{2}$ and $c_{2}+c_{3} \leq 1$. Then:

(1) If $c_{2}-c_{3} \leq 1-c_{1}$, then $\max _{\alpha \in\left[0,1-c_{1}\right]} g(\alpha)=g\left(1-c_{1}\right)=\frac{2-2 c_{1}+c_{2}}{3}$.

(2) If $c_{2}-c_{3}>1-c_{1}$, then $\max _{\alpha \in\left[0,1-c_{1}\right]} g(\alpha)=g\left(1-c_{1}\right)=\frac{3-3 c_{1}+3 c_{2}-c_{3}}{6}$.

Proof. By $c_{3}<c_{1}+c_{2}$ and $c_{2}+c_{3} \leq 1$, we obtain $\frac{c_{2}-c_{1}+c_{3}}{2}<c_{2}-c_{1}+c_{3} \leq 1-c_{1}$. Now (1) and (2) follow from Lemmas 4.8-4.10.

Lemma 4.18. Let $c_{3}<1<c_{1}+c_{2}, c_{2}+c_{3}>1$, and $c_{[3]} \leq 2$. Then:

(1) If $c_{3}<c_{1}$ and $c_{2}-c_{3} \leq 1-c_{1}$, then $\max _{\alpha \in\left[0,1-c_{1}\right]} g(\alpha)=g\left(1-c_{1}\right)=$ $\min \left\{\frac{2-2 c_{1}+c_{2}}{3}, \frac{2-c_{1}-c_{3}}{2}\right\}$.

(2) If $c_{3}<c_{1}$ and $c_{2}-c_{3}>1-c_{1}$, then $\max _{\alpha \in\left[0,1-c_{1}\right]} g(\alpha)=g\left(1-c_{1}\right)=$ $\min \left\{\frac{3-3 c_{1}+3 c_{2}-c_{3}}{6}, \frac{2-c_{1}-c_{3}}{2}\right\}$.

(3) If $c_{3} \geq c_{1}$, then $\max _{\alpha \in\left[0,1-c_{1}\right]} g(\alpha)=g\left(1-c_{1}\right)=\frac{2-c_{1}-c_{3}}{2}$.

Proof. From $c_{3}<c_{1}+c_{2}, c_{2}+c_{3}>1$, and $c_{[3]} \leq 2$, it follows that $0<$ $\frac{c_{2}-c_{1}+c_{3}}{2} \leq 1-c_{1}<c_{2}-c_{1}+c_{3}$. Then the statements follow from Lemmas 4.8-4.10.

Lemma 4.19. Let $c_{3}<1<c_{1}+c_{2}$ and $c_{[3]}>2$. Then: 
(1) If $c_{3}<c_{1}$ and $c_{2}-c_{3} \leq 1-c_{1}$, then $\max _{\alpha \in\left[0,1-c_{1}\right]} g(\alpha)=g\left(1-c_{1}\right)=$ $\min \left\{\frac{2-2 c_{1}+c_{2}}{3}, \frac{2-c_{1}+2 c_{2}-c_{3}}{6}\right\}$.

(2) If $c_{3}<c_{1}$ and $c_{2}-c_{3}>1-c_{1}$, then $\max _{\alpha \in\left[0,1-c_{1}\right]} g(\alpha)=g\left(1-c_{1}\right)=$ $\min \left\{\frac{3-3 c_{1}+3 c_{2}-c_{3}}{6}, \frac{2-c_{1}+2 c_{2}-c_{3}}{6}\right\}$.

(3) If $c_{3} \geq c_{1}$, then $\max _{\alpha \in\left[0,1-c_{1}\right]} g(\alpha)=g\left(1-c_{1}\right)=\frac{2-c_{1}+2 c_{2}-c_{3}}{6}$.

Proof. From $c_{3}<c_{1}+c_{2}$ and $c_{[3]}>2$, it follows that $0<1-c_{1}<\frac{c_{2}-c_{1}+c_{3}}{2} \leq$ $c_{2}-c_{1}+c_{3}$. Then the statements follow from Lemmas 4.8-4.10.

Based on Lemmas 4.16-4.19, we obtain all Nash equilibria for the case $c_{1}+c_{2}>1$.

Theorem 4.20. Let $c_{1}+c_{2}>1$.

(1) If $c_{2}+c_{3} \leq 1$, then:

(1.1) If $c_{2}-c_{3} \leq 1-c_{1}$, then every $\alpha \in\left[\max \left\{0, \frac{4-4 c_{1}-c_{2}}{3}\right\}, 1-c_{1}\right]$ results in a Nash equilibrium.

(1.2) If $c_{2}-c_{3}>1-c_{1}$, then every $\alpha \in\left[\max \left\{0, \frac{3-3 c_{1}-c_{3}}{3}\right\}, 1-c_{1}\right]$ results in a Nash equilibrium.

(2) If $c_{2}+c_{3}>1$ and $c_{[3]} \leq 2$, then:

(2.1) If $c_{3}<c_{1}$ and $c_{2}-c_{3} \leq 1-c_{1}$ and $4 c_{1}+c_{2} \leq 4$, then every $\alpha \in$ $\left[\min \left\{\frac{1-4 c_{1}+2 c_{2}+3 c_{3}}{3}, 1-c_{1}\right\}, 1-c_{1}\right]$ results in a Nash equilibrium.

(2.2) If $c_{3}<c_{1}, c_{2}-c_{3} \leq 1-c_{1}$, and $4 c_{1}+c_{2}>4$, then every $\alpha \in$ $\left[\max \left\{0,3-4 c_{1}+c_{3}\right\}, 1-c_{1}\right]$ results in a Nash equilibrium.

(2.3) If $c_{3}<c_{1}, c_{2}-c_{3}>1-c_{1}$, and $3 c_{1}+c_{3} \leq 3$, then every $\alpha \in$ $\left[\min \left\{\frac{3 c_{2}-3 c_{1}+2 c_{3}}{3}, 1-c_{1}\right\}, 1-c_{1}\right]$ results in a Nash equilibrium.

(2.4) If $c_{3}<c_{1}, c_{2}-c_{3}>1-c_{1}$, and $3 c_{1}+c_{3}>3$, then every $\alpha \in$ $\left[\max \left\{0,2-3 c_{1}+c_{2}\right\}, 1-c_{1}\right]$ results in a Nash equilibrium.

(2.5) If $c_{3} \geq c_{1}$, then $\mu$ is the only Nash equilibrium.

(3) If $c_{[3]}>2$, then: 
(3.1) Every $\alpha \in\left[\min \left\{\max \left\{0,3-4 c_{1}+c_{3}\right\}, 1-c_{1}\right\}, 1-c_{1}\right]$ results in a Nash equilibrium if $c_{3}<c_{1}$ and $c_{2}-c_{3} \leq 1-c_{1}$.

(3.2) Every $\alpha \in\left[\min \left\{\max \left\{0,2-3 c_{1}+c_{2}\right\}, 1-c_{1}\right\}, 1-c_{1}\right]$ results in $a$ Nash equilibrium if $c_{3}<c_{1}$ and $c_{2}-c_{3}>1-c_{1}$.

(3.3) If $c_{3} \geq c_{1}$, then $\mu$ is the only Nash equilibrium.

\section{Appendix C Proof of Proposition 4.3}

We assume that all strategies (and actions) of any player $j$ are in $\widetilde{\mathscr{S}_{j}}$.

The crucial subgames in the analysis are those subgames for $\{3,4\}$ where player 2 has not acted myopically.

Lemma 4.21. Let $n=4$, and let player 4 act myopically at every subgame for \{4\}. Suppose that in every subgame for $\{3,4\}$ where player 2 has not acted myopically, acting myopically is a best reply of player 3 . Then $\mu$ is a subgame perfect equilibrium.

Proof. By Lemma 4.1, $\mu_{4}$ is a best reply in every subgame for player 4. By Lemmas 4.4 and $4.5, \mu_{2}$ is a best reply against $\left(\mu_{3}, \mu_{4}\right)$. In every subgame for $\{3,4\}$ after player 2 has acted myopically, $\mu_{3}$ is a best reply against $\mu_{4}$, again by Lemmas 4.4 and 4.5. By the assumption in the lemma, this is also true if player 2 has not acted myopically. Hence, $\mu$ is a subgame perfect equilibrium.

We describe these subgames for $\{3,4\}$ where player 2 has not acted myopically, conveniently, by pairs $\left(x_{1}, x_{2}\right)$, where

$$
0<x_{1} \leq c_{1} \leq x_{2}<1, x_{1} \leq c_{2}, x_{1}+x_{2}=c_{1}+c_{2}
$$

or, equivalently

$$
0<x_{1} \leq c_{1}, x_{1} \leq c_{2}, x_{1}>c_{1}+c_{2}-1, x_{1}+x_{2}=c_{1}+c_{2} .
$$

In words, the part of the interval up to $x_{1}$ is claimed by both players 1 and 2 , the part between $x_{1}$ and $x_{2}$ is claimed by only one player, and the part between $x_{2}$ and 1 is claimed by no player. (Cf. Figure 4.7.) 


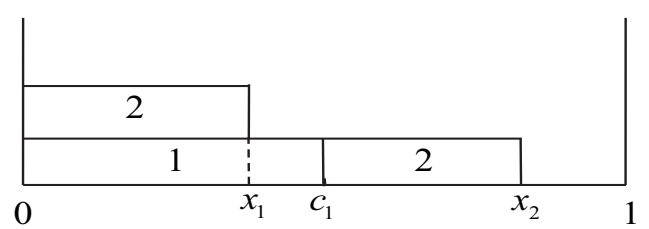

FIGURE 4.7: Subgames for $\{3,4\}$ where player 2 has not acted myopically

Recall that $r=\left(c_{1}+c_{2}+c_{3}+c_{4}\right) \bmod 1$.

Lemma 4.22. Let $\left(x_{1}, x_{2}\right)$, satisfying (4.5), be a subgame for $\{3,4\}$, and let player 4 always act myopically. Then acting myopically is not optimal for player 3 if, and only if, the following conditions hold:

(a) $x_{1}<c_{1}+c_{2}+c_{3}-1$.

(b) If $c_{1}+c_{2}+c_{3}+c_{4} \leq 2$, then $x_{1}>1-c_{3}-c_{4}$.

(c) If $c_{1}+c_{2}+c_{3}+c_{4}>2$, then

(c.1) $c_{4}<1-x_{1}$

(c.2) If $c_{3}>1-x_{1}$, then $c_{1}+c_{2}+\frac{2}{3} c_{3}+c_{4}-\frac{5}{3}<x_{1}<3-c_{1}-c_{2}-3 c_{4}$ or $x_{1} \geq c_{1}+c_{2}+c_{4}-1$.

(c.3) If $c_{3} \leq 1-x_{1}$, then $\frac{3}{2} r<x_{1}<c_{1}+c_{2}+c_{3}-1-\frac{3}{2} r$.

Proof. We start with the only-if direction. Assume that player 3's action is optimal, and that player 3's myopic action is not optimal.

We first show that player 3 always claims the part $\left[x_{2}, \min \left\{x_{2}+c_{3}, 1\right\}\right]$. Suppose this were not true, i.e., player 3 claims $\left[x_{2}, x_{2}+\alpha\right]$ where $0 \leq \alpha<$ $\min \left\{c_{3}, 1-x_{2}\right\}$. Then it must be the case that player 3 claims no part of $\left[0, x_{1}\right]$, since otherwise taking away a small amount $\varepsilon>0$ from such a claim and claiming $\left[x_{2}, x_{2}+\alpha+\varepsilon\right]$ instead, results in a gain of at least $\frac{\varepsilon}{2}$ and a loss of at most $\frac{\varepsilon}{3}$, contradicting the optimality of player 3's action. But then, player 3 's myopic action (claiming $\left[x_{2}, \min \left\{x_{2}+c_{3}, 1\right\}\right]$ and $\left[x_{1}, x_{1}+\beta\right]$ for some $\beta \geq 0$ ) results in a gain of at least $\frac{\varepsilon}{2}$ and a loss of at most $\frac{\varepsilon}{2}$, which means that this action is optimal, contradicting our assumption. 
Therefore we must have $c_{3}>1-x_{2}$, otherwise acting myopically and claiming $\left[x_{2}, x_{2}+c_{3}\right]$ is optimal for player 3 . The inequality $c_{3}>1-x_{2}$ is, by (4.5), equivalent to (a).

The premiss in (b) is equivalent to the inequality $c_{3}-\left(1-x_{2}\right)+c_{4} \leq 1-x_{1}$, as follows from (4.5). Since player 3 has already claimed $\left[x_{2}, 1\right]$, the lefthand side of this inequality is the remaining entitlement of player 3 plus the entitlement of player 4 . If this were smaller than or equal to $x_{2}-x_{1}$, then it would be optimal for player 3 to act myopically. Therefore, we must have $c_{3}-\left(1-x_{2}\right)+c_{4}>x_{2}-x_{1}$, which is equivalent to the consequence in (b).

The premiss in (c) is equivalent to the inequality $c_{3}-\left(1-x_{2}\right)+c_{4}>1-x_{1}$. Acting non-myopically can then only be optimal for player 3 if player 4 cannot fully claim the part of $\left[x_{1}, 1\right]$ that is not claimed by player 3 . This means that $c_{4}<1-x_{1}$, which is (c. 1$)$.

Suppose that the premiss in (c.2) holds, i.e., $c_{3}>1-x_{1}$. In that case, if player 3 would act myopically, he would claim the part $\left[x_{1}, 1\right]$ plus the part $\left[0, c_{3}-1+x_{1}\right]$, and have final payoff

$$
\frac{c_{3}-\left(1-x_{1}\right)}{3}+\frac{1-x_{1}}{2} .
$$

Let $\alpha=\max \left\{c_{4}-\left(1-x_{2}\right), 0\right\}$ be the entitlement of player 4 remaining after claiming (part of) $\left[x_{2}, 1\right]$. Improving on the payoff in (4.6) is only possible for player 3 by transferring a claim of size $\alpha+\varepsilon$ from $\left[x_{1}, x_{2}\right]$ for some $\varepsilon>0$ and adding it to $\left[0, c_{3}-1+x_{1}\right]$, resulting in a payoff

$$
\frac{c_{3}-\left(1-x_{1}\right)}{3}+\frac{\alpha+\varepsilon}{3}+\varepsilon+\frac{c_{3}-\left(c_{3}-\left(1-x_{1}\right)+\alpha+\varepsilon+\varepsilon\right)}{2},
$$

which is larger than the myopic payoff in (4.6) if and only if $\varepsilon>\frac{\alpha}{2}$. This transfer of claim is feasible if $\alpha=0$, i.e., if $c_{4} \leq 1-x_{2}$, or if $\alpha>0$ and $\frac{3}{2}\left(c_{4}-\left(1-x_{2}\right)\right)<\min \left\{x_{2}-x_{1}, 1-c_{3}\right\}$. This is equivalent to the consequences in (c.2).

Finally, suppose that the premiss in (c.3) holds, i.e., $c_{3} \leq 1-x_{1}$. In this case, if player 3 acts myopically, he receives payoff $\frac{c_{3}}{2}$. In this case, if player 3 can transfer part of his claim on $\left[x_{1}, x_{2}\right]$, namely an amount $r+\varepsilon$ for some 
$\varepsilon>0$, to $[0, r+\varepsilon]$, his payoff becomes

$$
\frac{r+\varepsilon}{3}+\varepsilon+\frac{c_{3}-r-2 \varepsilon}{2}
$$

which is higher than the myopic payoff if and only if $\varepsilon>\frac{r}{2}$. Such a transfer is possible if $c_{3}-\left(1-x_{2}\right)>\frac{3}{2} r$ and $x_{1}>\frac{3}{2} r$. This is equivalent to the consequence in (c.3).

Since the arguments for the if-part are similar to the arguments used for the only-if part, we just outline them. Suppose that (a), (b), and (c) hold. Then $c_{3}>1-x_{2}$ by (a). We distinguish the following cases.

(i) Suppose that $c_{1}+c_{2}+c_{3}+c_{4} \leq 2$, or, equivalently, $c_{3}-\left(1-x_{2}\right)+c_{4} \leq$ $1-x_{1}$, and hence $x_{1}>1-c_{3}-c_{4}$ by (b). Compared to myopic play, player 3 improves by transferring a small part $\varepsilon$ of his claim from $\left[x_{1}, 1\right]$ to $[0, \varepsilon]$.

(ii) Suppose that $c_{1}+c_{2}+c_{3}+c_{4}>2$, or, equivalently, $c_{3}-\left(1-x_{2}\right)+c_{4}>$ $1-x_{1}$, and hence $c_{4}<1-x_{1}$ by (c. 1 ).

(ii.1) Suppose $c_{3}>1-x_{1}$ and $c_{1}+c_{2}+\frac{2}{3} c_{3}+c_{4}-\frac{5}{3}<x_{1}<3-c_{1}-c_{2}-3 c_{4}$ (this is the first case in (c.2)). In this case, acting myopically yields the payoff in (4.6) to player 3, but player 3 can achieve the higher payoff in (4.7) by transferring a part of his claim as described before (4.7).

(ii.2) Suppose $c_{3}>1-x_{1}$ and $x_{1} \geq c_{1}+c_{2}+c_{4}-1$ (this is the second case in (c.2)). In the same way as in (ii.1), player 3 can improve upon myopic play.

(ii.3) Suppose $c_{3} \leq 1-x_{1}$, and hence $\frac{3}{2} r<x_{1}<c_{1}+c_{2}+c_{3}-1-\frac{3}{2} r$ by (c.3). Then player 3 can achieve the payoff in (4.8), which is higher than the myopic payoff $\frac{c_{3}}{2}$.

Proof of Proposition 4.3. By Lemma 4.21 it is sufficient to consider the actions of player 3 in subgames for $\{3,4\}$ such that player 2 has not acted myopically, i.e., subgames of the form $\left(x_{1}, x_{2}\right)$ satisfying the conditions in (4.5). By Lemma 4.22 it follows that $\mu$ is a subgame perfect equilibrium if and only if for each such subgame at least one of the conditions (a), (b), and (c) in Lemma 4.22 does not hold. 
(i) Suppose $c_{1}+c_{2}+c_{3}+c_{4} \leq 2$. Then, by (a) and (b) in Lemma 4.22, $\mu$ is a subgame perfect equilibrium exactly if there is no $x_{1}$ satisfying (4.5), and $1-c_{3}-c_{4}<x_{1}<c_{1}+c_{2}+c_{3}-1$. This is the case if and only if

$$
\max \left\{0, c_{1}+c_{2}-1,1-c_{3}-c_{4}\right\} \geq \min \left\{c_{1}, c_{2}, c_{1}+c_{2}+c_{3}-1\right\} .
$$

Since $c_{1}+c_{2}-1 \leq 1-c_{3}-c_{4}$, the condition in (i) follows.

(ii) Suppose $c_{1}+c_{2}+c_{3}+c_{4}>2$. Then $\mu$ is a subgame perfect equilibrium exactly if there is no $x_{1}$ satisfying (4.5), (a), (c.1), and (c.2) or (c.3), in Lemma 4.22. This results in three conditions which should all be satisfied.

(ii.1) To guarantee that there is no $x_{1}$ satisfying the first possibility in (c.2), it is necessary and sufficient that $\max \left\{0, c_{1}+c_{2}-1,1-c_{3}, c_{1}+c_{2}+\right.$ $\left.\frac{2}{3} c_{3}+c_{4}-\frac{5}{3}\right\} \geq \min \left\{c_{1}, c_{2}, c_{1}+c_{2}+c_{3}-1,1-c_{4}, 3-c_{1}-c_{2}-3 c_{4}\right\}$. Since $0<1-c_{3}, c_{1}+c_{2}-1<c_{1}+c_{2}+\frac{2}{3} c_{3}+c_{4}-\frac{5}{3}$, and $c_{1}+c_{2}+c_{3}-1>1-c_{4}$, the condition in (ii.1) follows.

(ii.2) To guarantee that there is no $x_{1}$ satisfying the second possibility in (c.2), it is necessary and sufficient that $\max \left\{0, c_{1}+c_{2}-1,1-c_{3}, c_{1}+c_{2}+\right.$ $\left.c_{4}-1\right\} \geq \min \left\{c_{1}, c_{2}, c_{1}+c_{2}+c_{3}-1,1-c_{4}\right\}$. Since $0<1-c_{3}, c_{1}+c_{2}-1<$ $c_{1}+c_{2}+c_{4}-1,1-c_{3}<c_{1}+c_{2}+c_{4}-1$, and $c_{1}+c_{2}+c_{3}-1>1-c_{4}$, the condition in (ii.2) follows.

(ii.3) To guarantee that there is no $x_{1}$ satisfying (c.3), it is necessary and sufficient that $\max \left\{0, c_{1}+c_{2}-1, \frac{3}{2} r\right\} \geq \min \left\{c_{1}, c_{2}, c_{1}+c_{2}+c_{3}-1,1-c_{3}, 1-\right.$ $\left.c_{4}, c_{1}+c_{2}+c_{3}-1-\frac{3}{2} r\right\}$. Since $0<\frac{3}{2} r$, the condition in (ii.3) follows.

\section{Appendix D Proof of Theorem 4.7.}

We have to show that $\hat{\mu}$ is a subgame perfect equilibrium in $\mathscr{S}$.

Let $j \in[n]$ and, throughout the proof, fix an arbitrary claims profile for $[j-1]$, with numbers of claims $\beta_{i}, i \in[j-1]$. Let $\hat{r}$ be the red card player of this claims profile, possibly $\hat{r}=0$. We have to show that for player $j$ the $\operatorname{lrm}-\hat{r}$ action is a best reply against all players $h>j$ playing according to $\hat{\mu}_{h}$.

If $j=1$, then $\hat{r}=0$. Any action of player 1 is myopic and, by definition of $\hat{\mu}_{2}, \ldots \hat{\mu}_{n}$, results in the same payoff for player 1 as playing lrm-0, i.e., 
claiming $\left[0, c_{1}\right]$. So, in particular, $1 \mathrm{rm}-0$ is a best reply.

Now let $j \geq 2$. Without loss of generality we may assume that the claims profile for $[j-1]$ is decreasing, otherwise we can rearrange the claims of the players in $[j-1]$ (this may effect the payoffs of the players in $[j-1]$, but not those of the later players).

We first prove that if $j$ plays the lrm- $\hat{r}$ action, then $j$ 's payoff does not increase if every player $h>j$, instead of the lrm- $\hat{r}$ action, plays the $1 \mathrm{rm}-j$ action. We denote the strategy for player $h>j$, prescribing always the $1 \mathrm{rm}-j$ action, by $\bar{\mu}_{h}$. Let $\left(y^{\prime}, \beta^{\prime}, m^{\prime}\right)$ be the claims profile for $[n]$ resulting from $\left(\hat{\mu}_{i}\right)_{i \in\{j, \ldots, n\}}$ (i.e., player $j$ and all later players always playing the lrm- $\hat{r}$ action) and let $(\bar{y}, \bar{\beta}, \bar{m})$ be the claims profile for $[n]$ resulting from $\left(\hat{\mu}_{j},\left(\bar{\mu}_{i}\right)_{i \in\{j+1, \ldots, n\}}\right)$. Without loss of generality (otherwise rearrange) we may assume that $y^{\prime}=\bar{y}$ and $m^{\prime}=\bar{m}$, and we write $y$ and $m$ instead.

Claim 1. $u_{j}\left(y, \beta^{\prime}, m\right) \geq u_{j}(y, \bar{\beta}, m)$.

Proof. Clearly, $\bar{\beta}_{[j]}(t)=\beta_{[j]}^{\prime}(t)$ for all $t \in\{1, \ldots, m\}$. Since every $h>j$ plays the $\operatorname{lrm}-j$ action in $\bar{\beta}$ and plays the lrm- $\hat{r}$ action in $\beta^{\prime}$, it follows that for all $t \in\{1, \ldots, m\}$ with $\beta_{j}^{\prime}(t)=1$ (and hence $\bar{\beta}_{j}(t)=1$ ) and $\beta_{h}^{\prime}(t)=1$ we have $\bar{\beta}_{h}(t)=1$. Therefore, $\bar{\beta}_{[n]}(t) \geq \beta_{[n]}^{\prime}(t)$ for all $t \in\{1, \ldots, m\}$ with $\beta_{j}^{\prime}(t)=1$. This implies the inequality in Claim 1 .

By Claim 1 it is sufficient to show that for player $j$ the $1 \mathrm{rm}-\hat{r}$ action is a best reply against all later players $h$ playing according to $\bar{\mu}_{h}$, that is, playing always the lrm- $j$ action. Since the profile for $[j-1]$ is decreasing, we may also assume, by rearranging if necessary, that if player $j$ plays the $1 \mathrm{rm}-\hat{r}$ action, then for all $t, t^{\prime}$ with $t^{\prime}>t$ such that $\beta_{[j-1]}(t)=\beta_{[j-1]}\left(t^{\prime}\right)$ and $j$ claims $t^{\prime}$, then $j$ also claims $t$. (In words, if player $j$ plays the $1 \mathrm{rm}-\hat{r}$ action, then $j$ 's claims on each level of the claims profile for $[j-1]$ are ordered from left to right, without 'holes'. Hence, the $1 \mathrm{rm}-\hat{r}$ action leads to the claims profile for [j] resulting from $j$ playing left-right myopically.)

Suppose that player $j$ plays an action different from the $1 \mathrm{rm}-\hat{r}$ action. For the rest of this proof, we denote the resulting claims profile for $[n]$ by $(y, \beta, m)$, and let $M=\left\{t \in\{1, \ldots, m\}: \beta_{j}(t)=1\right\}$. The proof proceeds in two parts. In the first part (a) we show that if, on each level of $(y, \beta, m), j$ 
rearranges claims so that they are located from left to right (i.e., gets rid of 'holes'), then j's payoff does not change. In the second part (b) we show that if $j$, if possible, shifts claims to lower levels (which means, in particular, to the right of the interval), then again $j$ 's payoff does not decrease.

(a) Suppose there is $r_{0} \in\{1, \ldots, m\}$ such that $\beta_{[j-1]}\left(r_{0}\right)=\beta_{[j-1]}\left(r_{0}+1\right)$, $\beta_{j}\left(r_{0}\right)=0$, and $\beta_{j}\left(r_{0}+1\right)=1$. Without loss of generality we may assume that $y_{r_{0}+1}-y_{r_{0}}=y_{r_{0}}-y_{r_{0}-1}$ (otherwise appropriately refine the end partition). Let $\hat{\beta}$ be such that $\hat{\beta}_{i}=\beta_{i}$ for all $i \in[j-1], \hat{\beta}_{j}\left(r_{0}\right)=1, \hat{\beta}_{j}\left(r_{0}+1\right)=0$, $\hat{\beta}_{j}(t)=\beta_{j}(t)$ for all $t \neq r_{0}, r_{0}+1$, and $\hat{\beta}_{i}$, like $\beta_{i}$, is determined by $\bar{\mu}_{j+1}, \ldots, \bar{\mu}_{n}$ for all $i=j+1, \ldots, n$. (In words, the change from $\beta$ to $\hat{\beta}$ consists of player $j$ claiming $r_{0}$ instead of $r_{0}+1$, plus the consequence for the claims of the later players.) For this situation we prove the following claim.

Claim 2. $\hat{\beta}_{[n]}\left(r_{0}\right)=\beta_{[n]}\left(r_{0}+1\right), \hat{\beta}_{[n]}\left(r_{0}+1\right)=\beta_{[n]}\left(r_{0}\right)$, and $\hat{\beta}_{[n]}(t)=\beta_{[n]}(t)$ for all $t \neq r_{0}, r_{0}+1$ with $t \in M$.

Proof. If $\beta_{h}\left(r_{0}\right)=0$ for all $h \in[n]$ with $h>j$, then $\hat{\beta}_{[n]}\left(r_{0}\right)=\beta_{[j]}\left(r_{0}\right)+1=$ $\beta_{[j]}\left(r_{0}+1\right)=\beta_{[n]}\left(r_{0}+1\right), \hat{\beta}_{[n]}\left(r_{0}+1\right)=\beta_{[j]}\left(r_{0}+1\right)-1=\beta_{[j]}\left(r_{0}\right)=\beta_{[n]}\left(r_{0}\right)$, and $\hat{\beta}_{[n]}(t)=\beta_{[n]}(t)$ for all $t \neq r_{0}, r_{0}+1$ with $t \in M$.

Otherwise, let $h_{0}=\min \left\{h \in[n]: h>j, \beta_{h}\left(r_{0}\right)=1\right\}$. Then $h_{0}=\min \{h \in$ $\left.[n]: h>j, \hat{\beta}_{h}\left(r_{0}+1\right)=1\right\}$. Also, $\hat{\beta}_{\left[h_{0}\right]}(t)=\beta_{\left[h_{0}\right]}(t)$ for all $t \in M \backslash\left\{r_{0}, r_{0}+1\right\}$. Consider player $h_{0}+1$. Since this player plays the $1 \mathrm{mr}-j$ action, following the claims $\beta_{j}$ of player $j$, we have: if $\beta_{h_{0}+1}\left(r_{0}\right)=\beta_{h_{0}+1}\left(r_{0}+1\right)=0$ then $\hat{\beta}_{h_{0}+1}\left(r_{0}\right)=\hat{\beta}_{h_{0}+1}\left(r_{0}+1\right)=0$; if $\beta_{h_{0}+1}\left(r_{0}\right)=\beta_{h_{0}+1}\left(r_{0}+1\right)=1$ then $\hat{\beta}_{h_{0}+1}\left(r_{0}\right)=\hat{\beta}_{h_{0}+1}\left(r_{0}+1\right)=1$; if $\beta_{h_{0}+1}\left(r_{0}\right)=0$ and $\beta_{h_{0}+1}\left(r_{0}+1\right)=1$ then $\hat{\beta}_{h_{0}+1}\left(r_{0}\right)=1$ and $\hat{\beta}_{h_{0}+1}\left(r_{0}+1\right)=0$. The fourth case, $\beta_{h_{0}+1}\left(r_{0}\right)=1$ and $\beta_{h_{0}+1}\left(r_{0}+1\right)=0$, can only occur, due to the $1 \mathrm{mr}-j$ action of player $h_{0}+1$, if $\beta_{h_{0}}\left(r_{0}\right)=\beta_{h_{0}}\left(r_{0}+1\right)=1$ and hence $\beta_{\left[h_{0}\right]}\left(r_{0}\right)=\beta_{\left[h_{0}\right]}\left(r_{0}+1\right)-1$ and $\hat{\beta}_{\left[h_{0}\right]}\left(r_{0}\right)=\hat{\beta}_{\left[h_{0}\right]}\left(r_{0}+1\right)+1$; in that case, $\hat{\beta}_{h_{0}+1}\left(r_{0}\right)=0, \hat{\beta}_{h_{0}+1}\left(r_{0}\right)=1$, and $\hat{\beta}_{\left[h_{0}+1\right]}\left(r_{0}\right)=\hat{\beta}_{\left[h_{0}+1\right]}\left(r_{0}+1\right)=\beta_{\left[h_{0}+1\right]}\left(r_{0}\right)=\beta_{\left[h_{0}+1\right]}\left(r_{0}+1\right)$.

Hence, in all cases we have $\hat{\beta}_{\left[h_{0}+1\right]}\left(r_{0}\right)=\beta_{\left[h_{0}+1\right]}\left(r_{0}+1\right)$ and $\hat{\beta}_{\left[h_{0}+1\right]}\left(r_{0}+\right.$ $1)=\beta_{\left[h_{0}+1\right]}\left(r_{0}\right)$, and $\hat{\beta}_{\left[h_{0}+1\right]}(t)=\beta_{\left[h_{0}+1\right]}(t)$ for all $t \neq r_{0}, r_{0}+1$ with $t \in M$. By repeating this argument for the players $h_{0}+2, \ldots, n$, the statements in the Claim 2 follow. 
As a consequence of Claim 2, player $j$ 's payoff from changing his action by claiming $r_{0}$ instead of $r_{0}+1$ does not change. By performing consecutive changes like this, we may therefore assume, just keeping, with some abuse, the notation $\beta$, that the following holds: for all $t, t^{\prime} \in\{1, \ldots, m\}$, with $t^{\prime}>t$, if $\beta_{[j-1]}(t)=\beta_{[j-1]}\left(t^{\prime}\right)$ and $\beta_{j}\left(t^{\prime}\right)=1$, then $\beta_{j}(t)=1$. (In words, on each level of the claims profile for $[j-1]$ the claims of player $j$ are ordered from left to right without 'holes'.)

We next introduce notations, describing the situation reached in $(* *)$ in detail. Since the claims profile for $[j-1]$ is decreasing, there are $\left\{t_{0}, \ldots, t_{k}\right\} \subseteq$ $\{0, \ldots, m\}$ with $0=t_{0}<t_{1}<\cdots<t_{k}=m$ such that (i) $\beta_{[j-1]}(s)=\beta_{[j-1]}(t)$ for all $t_{\ell}<s, t \leq t_{\ell+1}$, for all $\ell=0, \ldots, k-1$; and (ii) $\beta_{[j-1]}(t)>\beta_{[j-1]}\left(t^{\prime}\right)$ for all $t, t^{\prime}$, and $\ell, \ell^{\prime} \in\{0, \ldots, k-1\}$ with $t_{\ell}<t \leq t_{\ell+1} \leq t_{\ell^{\prime}}<t^{\prime} \leq t_{\ell^{\prime}+1}$. (In words, this is a description of the levels of the decreasing claims profile for $[j-1]$.) Then by $(* *)$, for every $\ell=0, \ldots, k-1$, there is $t^{\prime}$ with $t_{\ell} \leq t^{\prime} \leq t_{\ell+1}$ such that $\beta_{j}(t)=1$ for every $t_{\ell}<t \leq t^{\prime}$ and $\beta_{j}(t)=0$ for every $t^{\prime}<t \leq t_{\ell+1}$. (In words, at every level of the claims profile for $[j-1]$, if player $j$ has claims between $t_{\ell}$ and $t_{\ell+1}$, that is, if $t^{\prime}>t_{\ell}$, then these claims are arranged from left to right without 'holes'.) With this profile for $[j]$ fixed, we now proceed with the final part of the proof, in which, possibly, claims of player $j$ are shifted to lower levels.

(b) Suppose there exist $s_{0}, s_{1} \in\{1, \ldots, m\}$ and $\ell \in\{0, \ldots, k-1\}$ such that $\beta_{[j-1]}\left(s_{1}\right)<\beta_{[j-1]}\left(s_{0}\right)$ (and hence $\left.s_{1}>s_{0}\right), s_{1} \in\left\{t_{\ell-1}+1, \ldots, t_{\ell}\right\}$, and such that $\beta_{j}\left(s_{0}\right)=1$ and $\beta_{j}\left(s_{1}\right)=0$. Without loss of generality we assume that $y_{t}-y_{t-1}=y_{t^{\prime}}-y_{t^{\prime}-1}$ for all $t, t^{\prime} \in\{1, \ldots, m\}$ (otherwise, since payoffs depend continuously on the lengths of the intervals, we may refine the end partition such that it becomes arbitrarily close to a partition with all intervals equal). Also, we can take $s_{1}$ such that, first, $\beta_{j}(t)=1$ for all $t_{\ell-1}+1 \leq t<s_{1}$, or $s_{1}=t_{\ell-1}+1$ if $\beta_{j}(t)=0$ for all $t_{\ell-1}+1 \leq t \leq t_{\ell}$, and, second, $\beta_{j}(t)=1$ for all $t>t_{\ell}$. (In words, we can take $s_{1}$ such that player $j$ claims everything to the left of $s_{1}$ at the same level, and, moreover, every lower level interval.) Moreover, we can take $s_{0}$ such that $\beta_{j}(t)=0$ for all $t<s_{0}$. (In words, we take $s_{0}$ such that player $j$ claims nothing to the left of $s_{0}$, i.e., $s_{0}$ is the left most interval claimed by $j$ at $\beta$.) 
Let $\widetilde{\beta}$ be such that $\widetilde{\beta}_{i}=\beta_{i}$ for all $i \in[j-1], \widetilde{\beta}_{j}\left(s_{0}\right)=0, \widetilde{\beta}_{j}\left(s_{1}\right)=1$, $\widetilde{\beta}_{j}(t)=\beta_{j}(t)$ for all $t \neq s_{0}, s_{1}$, and $\widetilde{\beta}_{i}$ is determined by $\bar{\mu}_{j+1}, \ldots, \bar{\mu}_{n}$ for all $i=j+1, \ldots, n$. (In words, player $j$ claims $s_{1}$ instead of $s_{0}$.)

Claim 3. $\widetilde{\beta}_{[n]}\left(s_{1}\right) \leq \beta_{[n]}\left(s_{0}\right)$, and $\widetilde{\beta}_{[n]}(t)=\beta_{[n]}(t)$ for all $t \in M$ with $t>s_{1}$. Proof. Since $\tilde{\beta}_{[j-1]}\left(s_{0}\right)>\widetilde{\beta}_{[j-1]}\left(s_{1}\right), \widetilde{\beta}_{j}\left(s_{0}\right)=0$ and $\widetilde{\beta}_{j}\left(s_{1}\right)=1$, we have $\tilde{\beta}_{[j]}\left(s_{0}\right) \geq \widetilde{\beta}_{[j]}\left(s_{1}\right)$. Hence, since every $h>j$ plays the lrm-j action, and therefore, in particular, myopically, we have $\widetilde{\beta}_{[n]}\left(s_{1}\right) \leq \widetilde{\beta}_{[n]}\left(s_{0}\right)+1$. Also, $\widetilde{\beta}_{[n]}\left(s_{0}\right) \leq \beta_{[n]}\left(s_{0}\right)$. We consider two cases. (i) If $\widetilde{\beta}_{[n]}\left(s_{1}\right) \leq \widetilde{\beta}_{[n]}\left(s_{0}\right)$, then $\widetilde{\beta}_{[n]}\left(s_{1}\right) \leq \beta_{[n]}\left(s_{0}\right)$. (ii) Otherwise, $\widetilde{\beta}_{[n]}\left(s_{1}\right)=\widetilde{\beta}_{[n]}\left(s_{0}\right)+1$, but in that case $\widetilde{\beta}_{[n]}\left(s_{0}\right)=\beta_{[n]}\left(s_{0}\right)-1$, so that $\widetilde{\beta}_{[n]}\left(s_{1}\right)=\widetilde{\beta}_{[n]}\left(s_{0}\right)+1=\beta_{[n]}\left(s_{0}\right)$. This proves the first statement in Claim 3.

Since every $h>j$ plays the lrm-j action, we have $\widetilde{\beta}_{h}(t)=\beta_{h}(t)$ for all $t>s_{1}$ with $t \in M$. Together with $\widetilde{\beta}_{[j]}(t)=\beta_{[j]}(t)$, we have $\widetilde{\beta}_{[n]}(t)=\beta_{[n]}(t)$ for all $t>s_{1}$ with $t \in M$. This is the second statement in the claim. Hence, the proof of Claim 3 is now complete.

The following claim is an intermediate result used in the final part below. It says that, when going from $\beta$ to $\widetilde{\beta}$, there is at most one interval on which the total claim is increased (by one).

Claim 4. Let $M_{n}^{\prime}=\left\{t \in\{1, \ldots, m\}: \widetilde{\beta}_{[n]}(t)=\beta_{[n]}(t)+1\right\}$. Then $\left|M_{n}^{\prime}\right| \leq 1$.

Proof. We use induction on the number of players. For $n=j$, the claim holds since by definition of $\beta$ and $\widetilde{\beta}$ we have $M_{j}^{\prime}=\left\{s_{1}\right\}$. Assume that $\left|M_{h^{\prime}}^{\prime}\right| \leq 1$ for all $j \leq h^{\prime} \leq h$ for some $j<h<n$. We prove that $\left|M_{h+1}^{\prime}\right| \leq 1$. We distinguish two cases.

(1) $\left|M_{h}^{\prime}\right|=0$. In this case, $\widetilde{\beta}_{[h]}(t)=\beta_{[h]}(t)$ for all $t \in\{1, \ldots, m\}$. The lrm$j$ action of player $h+1$ results in a sequence of claims, starting with the least claimed intervals, giving precedence to the intervals claimed by $j$, and from left to right without 'holes'. Exactly one of the following two claiming sequences will result.

- $M_{1} \rightarrow\left\{s_{1}\right\} \rightarrow M_{2} \rightarrow\left\{s_{0}\right\} \rightarrow M_{3}$. Here, $M_{1}, M_{2}, M_{3},\left\{s_{0}\right\},\left\{s_{1}\right\}$ form a partition of $\{1, \ldots, m\}$. Player $h+1$ first claims intervals in $M_{1}$, next (if there is some entitlement of the total $c_{h+1}$ left) $s_{1}$, next intervals from $M_{2}$, etc, until $c_{h+1}$ is exhausted. 
- $M_{1} \rightarrow\left\{s_{0}\right\} \rightarrow M_{2} \rightarrow\left\{s_{1}\right\} \rightarrow M_{3}$, with a similar interpretation.

We only consider the first claiming sequence, the other one is similar. There are essentially three possibilities, where we use the assumption that all intervals have equal length, denoted by $\tau$. Also, the number of intervals in some set $M_{i}$ is denoted by $m_{i}$.

(1.1) $c_{h+1} \leq m_{1} \tau$. Then $\widetilde{\beta}_{[h+1]}(t)=\beta_{[h+1]}(t)$ for all $t \in\{1, \ldots, m\}$. Thus, $M_{h+1}^{\prime}=\emptyset$.

(1.2) $\left(m_{1}+1\right) \tau \leq c_{h+1} \leq\left(m_{1}+m_{2}+1\right) \tau$. If $\widetilde{\beta}_{h+1}\left(s_{1}\right)=\beta_{h+1}\left(s_{1}\right)$, we have $M_{h+1}^{\prime}=\emptyset$. If $\widetilde{\beta}_{h+1}\left(s_{1}\right)=1$ and $\beta_{h+1}\left(s_{1}\right)=0$, then $M_{h+1}^{\prime}=\left\{s_{1}\right\}$.

(1.3) $\left(m_{1}+m_{2}+2\right) \tau \leq c_{h+1} \leq\left(m_{1}+m_{2}+m_{3}+2\right) \tau$. If $\widetilde{\beta}_{h+1}\left(s_{1}\right)=\beta_{h+1}\left(s_{1}\right)$ and $\widetilde{\beta}_{h+1}\left(s_{0}\right)=\beta_{h+1}\left(s_{0}\right)$, then $M_{h+1}^{\prime}=\emptyset$. If $\widetilde{\beta}_{h+1}\left(s_{1}\right)=\beta_{h+1}\left(s_{1}\right), \widetilde{\beta}_{h+1}\left(s_{0}\right)=$ 0 and $\beta_{h+1}\left(s_{0}\right)=1$, then $M_{h+1}^{\prime}$ consists of the last interval claimed by player $h+1$ according to $\widetilde{\beta}$. If $\widetilde{\beta}_{h+1}\left(s_{1}\right)=1$ and $\beta_{h+1}\left(s_{1}\right)=0$, we have $M_{h+1}^{\prime}=\left\{s_{1}\right\}$.

(2) $\left|M_{h}^{\prime}\right|=1$. Suppose that $M_{h}^{\prime}=\left\{s_{1}^{\prime}\right\}$. Then there is an interval $s_{0}^{\prime}$ with $\widetilde{\beta}_{[n]}\left(s_{0}^{\prime}\right)=\beta_{[n]}\left(s_{0}^{\prime}\right)-1$, and $\widetilde{\beta}_{[h]}(t)=\beta_{[h]}(t)$ and $\widetilde{\beta}_{j}(t)=\beta_{j}(t)$ for all $t \in$ $\{1, \ldots, m\} \backslash\left\{s_{0}, s_{0}^{\prime}, s_{1}, s_{1}^{\prime}\right\}$. Thus, there are 4 ! claiming sequences for player $h+1$. We only consider the sequence $M_{1} \rightarrow s_{1}^{\prime} \rightarrow M_{2} \rightarrow s_{1} \rightarrow M_{3} \rightarrow s_{0}^{\prime} \rightarrow$ $M_{4} \rightarrow s_{0} \rightarrow M_{5}$ with $s_{0}, s_{0}^{\prime}, s_{1}, s_{1}^{\prime} \notin M_{i}, i=1, \ldots, 5$ (the arguments for the other sequences are again similar). We have the following possibilities.

$c_{h+1} \leq m_{1} \tau$. Then $M_{h+1}^{\prime}=M_{h}^{\prime}$.

(2.2) $\left(m_{1}+1\right) \leq c_{h+1} \leq\left(m_{1}+m_{2}+1\right) \tau$. If $\widetilde{\beta}_{h+1}\left(s_{1}^{\prime}\right)=\beta_{h+1}\left(s_{1}^{\prime}\right)=1$, then $M_{h+1}^{\prime}=M_{h}^{\prime}$. If $\widetilde{\beta}_{h+1}\left(s_{1}^{\prime}\right)=0$ and $\beta_{h+1}\left(s_{1}^{\prime}\right)=1$, then $M_{h+1}^{\prime}$ consists of the last interval claimed by player $h+1$ according to $\widetilde{\beta}$.

(2.3) $\left(m_{1}+m_{2}+2\right) \tau \leq c_{h+1} \leq\left(m_{1}+m_{2}+m_{3}+2\right) \tau$. If $\widetilde{\beta}_{h+1}\left(s_{1}^{\prime}\right)=\beta_{h+1}\left(s_{1}^{\prime}\right)$, then $\widetilde{\beta}_{h+1}\left(s_{1}\right)=\beta_{h+1}\left(s_{1}\right)$ by the lrm-j action of player $h+1$ and thus, $M_{h+1}^{\prime}=\left\{s_{1}^{\prime}\right\}$. If $\widetilde{\beta}_{h+1}\left(s_{1}^{\prime}\right)=0, \beta_{h+1}\left(s_{1}^{\prime}\right)=1$ and $\widetilde{\beta}_{h+1}\left(s_{1}\right)=\beta_{h+1}\left(s_{1}\right)$, then $M_{h+1}^{\prime}$ consists of the last interval claimed by $h+1$ according to $\widetilde{\beta}$. If $\widetilde{\beta}_{h+1}\left(s_{1}^{\prime}\right)=0, \beta_{h+1}\left(s_{1}^{\prime}\right)=1, \widetilde{\beta}_{h+1}\left(s_{1}\right)=1$ and $\beta_{h+1}\left(s_{1}\right)=0$, then $M_{h+1}^{\prime}=\left\{s_{1}\right\}$. 
(2.4) $\left(m_{1}+m_{2}+m_{3}+3\right) \tau \leq c_{h+1} \leq\left(m_{1}+\cdots+m_{4}+3\right) \tau$. If $\widetilde{\beta}_{h+1}\left(s_{1}^{\prime}\right)=\beta_{h+1}\left(s_{1}^{\prime}\right)$ and $\widetilde{\beta}_{h+1}\left(s_{1}\right)=\beta_{h+1}\left(s_{1}\right)$, then $M_{h+1}^{\prime}=\left\{s_{1}^{\prime}\right\}$. If $\widetilde{\beta}_{h+1}\left(s_{1}^{\prime}\right)=0, \beta_{h+1}\left(s_{1}^{\prime}\right)=$ 1, $\widetilde{\beta}_{h+1}\left(s_{1}\right)=\beta_{h+1}\left(s_{1}\right)$ and $\widetilde{\beta}_{h+1}\left(s_{0}^{\prime}\right)=\beta_{h+1}\left(s_{0}^{\prime}\right)$, then $M_{h+1}^{\prime}$ consists of the last interval claimed by $h+1$ according to $\widetilde{\beta}$. If $\widetilde{\beta}_{h+1}\left(s_{1}^{\prime}\right)=0$, $\beta_{h+1}\left(s_{1}^{\prime}\right)=1, \widetilde{\beta}_{h+1}\left(s_{1}\right)=\beta_{h+1}\left(s_{1}\right), \widetilde{\beta}_{h+1}\left(s_{0}^{\prime}\right)=1$ and $\beta_{h+1}\left(s_{0}^{\prime}\right)=0$, then $M_{h+1}^{\prime}=\emptyset$. If $\widetilde{\beta}_{h+1}\left(s_{1}^{\prime}\right)=0, \beta_{h+1}\left(s_{1}^{\prime}\right)=1, \widetilde{\beta}_{h+1}\left(s_{1}\right)=1$ and $\beta_{h+1}\left(s_{1}\right)=0$, then $M_{h+1}^{\prime}=\left\{s_{1}\right\}$.

(2.5) $\left(m_{1}+\cdots+m_{4}+4\right) \tau \leq c_{h+1} \leq\left(m_{1}+\cdots+m_{5}+4\right) \tau$. If $\widetilde{\beta}_{h+1}\left(s_{1}^{\prime}\right)=\beta_{h+1}\left(s_{1}^{\prime}\right)$ and $\widetilde{\beta}_{h+1}\left(s_{1}\right)=\beta_{h+1}\left(s_{1}\right)$, then $M_{h+1}^{\prime}=\left\{s_{1}^{\prime}\right\}$. If $\widetilde{\beta}_{h+1}\left(s_{1}^{\prime}\right)=0, \beta_{h+1}\left(s_{1}^{\prime}\right)=$ $1, \widetilde{\beta}_{h+1}\left(s_{1}\right)=\beta_{h+1}\left(s_{1}\right)$ and $\widetilde{\beta}_{h+1}\left(s_{0}^{\prime}\right)=\beta_{h+1}\left(s_{0}^{\prime}\right)$, we have $\widetilde{\beta}_{h+1}\left(s_{0}\right)=$ $\beta_{h+1}\left(s_{0}\right)=1$. Then $M_{h+1}^{\prime}$ consists of the last interval claimed by $h+1$ according to $\widetilde{\beta}$. If $\widetilde{\beta}_{h+1}\left(s_{1}^{\prime}\right)=0, \beta_{h+1}\left(s_{1}^{\prime}\right)=1, \widetilde{\beta}_{h+1}\left(s_{1}\right)=\beta_{h+1}\left(s_{1}\right)$, $\widetilde{\beta}_{h+1}\left(s_{0}^{\prime}\right)=1, \beta_{h+1}\left(s_{0}^{\prime}\right)=0$ and $\widetilde{\beta}_{h+1}\left(s_{0}\right)=\beta_{h+1}\left(s_{0}\right)$, then $M_{h+1}^{\prime}=\emptyset$. If $\widetilde{\beta}_{h+1}\left(s_{1}^{\prime}\right)=0, \beta_{h+1}\left(s_{1}^{\prime}\right)=1, \widetilde{\beta}_{h+1}\left(s_{1}\right)=\beta_{h+1}\left(s_{1}\right), \widetilde{\beta}_{h+1}\left(s_{0}^{\prime}\right)=1$, $\beta_{h+1}\left(s_{0}^{\prime}\right)=0, \widetilde{\beta}_{h+1}\left(s_{0}\right)=0$ and $\beta_{h+1}\left(s_{0}\right)=1$, then $M_{h+1}^{\prime}$ consists of the last interval claimed by $h+1$ according to $\widetilde{\beta}$. If $\widetilde{\beta}_{h+1}\left(s_{1}^{\prime}\right)=0$, $\beta_{h+1}\left(s_{1}^{\prime}\right)=1, \widetilde{\beta}_{h+1}\left(s_{1}\right)=1$ and $\beta_{h+1}\left(s_{1}\right)=0$, then $M_{h+1}^{\prime}=\left\{s_{1}\right\}$.

This concludes the proof of Claim 4 .

Based on Claim 3, we consider two cases.

Case(i): $\widetilde{\beta}_{[n]}\left(s_{1}\right)=\beta_{[n]}\left(s_{0}\right)$.

We consider two subcases.

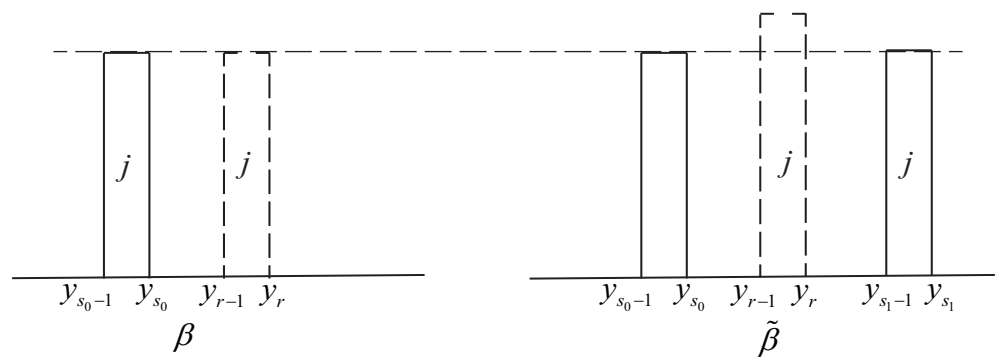

FIgURE 4.8: Case (i.1): $\widetilde{\beta}_{[n]}\left(s_{0}\right)=\beta_{[n]}\left(s_{0}\right)$

(i.1) $\widetilde{\beta}_{[n]}\left(s_{0}\right)=\beta_{[n]}\left(s_{0}\right)$. If there would be an $r \in M, s_{0}<r<s_{1}$, with $\widetilde{\beta}_{[n]}(r)=\widetilde{\beta}_{[n]}\left(s_{1}\right)+1$, then by the lrm-j actions of the players $h>j$ we would 
have $\widetilde{\beta}_{[n]}\left(s_{0}\right)=\beta_{[n]}\left(s_{0}\right)-1$. Therefore, $\widetilde{\beta}_{[n]}(t)=\widetilde{\beta}_{[n]}\left(s_{1}\right)$ for all $s_{0}<t<s_{1}$ with $t \in M$. (Cf. Figure 4.8.)

(i.2) Otherwise, $\widetilde{\beta}_{[n]}\left(s_{0}\right)+1=\beta_{[n]}\left(s_{0}\right)$. If there would be an $r^{\prime} \in M, s_{0}<r^{\prime}<$ $s_{1}$ with $\widetilde{\beta}_{[n]}\left(r^{\prime}\right) \geq \widetilde{\beta}_{[n]}\left(s_{1}\right)+1$, then $\widetilde{\beta}_{[n]}\left(r^{\prime}\right)-\widetilde{\beta}_{[n]}\left(s_{0}\right) \geq \widetilde{\beta}_{[n]}\left(s_{1}\right)-\widetilde{\beta}_{[n]}\left(s_{0}\right)+1=$ 2 , which contradicts with the myopic strategies of all players $h>j$. (Cf. Figure 4.9.) Hence, we again have $\widetilde{\beta}_{[n]}(t)=\widetilde{\beta}_{[n]}\left(s_{1}\right)$ for all $s_{0}<t<s_{1}$ with $t \in M$.

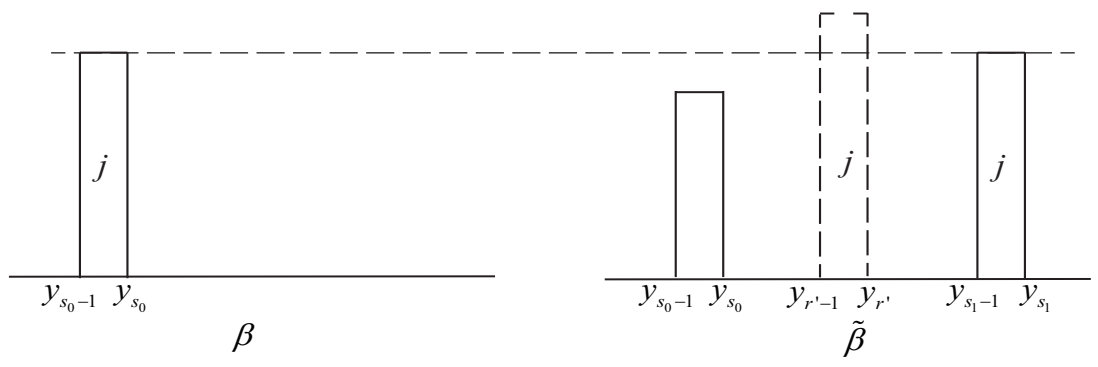

FIGURE 4.9: Case (i.2): $\widetilde{\beta}_{[n]}\left(s_{0}\right)=\beta_{[n]}\left(s_{0}\right)-1$

Now suppose that there would exist $s_{0}<p<s_{1}$ with $p \in M$ such that $\widetilde{\beta}_{[n]}(p)=\beta_{[n]}(p)+1$. Then, since $\widetilde{\beta}_{[n]}(t)=\widetilde{\beta}_{[n]}\left(s_{1}\right)$ for all $s_{0}<t<s_{1}$ with $t \in M$, we would have $\widetilde{\beta}_{[n]}(t)=\beta_{[n]}(t)+1$ for all $p<t \leq s_{1}$ (cf. Figure 4.10), but this contradicts Claim 4. Hence, $\widetilde{\beta}_{[n]}(t) \leq \beta_{[n]}(t)$ for all $s_{0}<t<s_{1}$ with $t \in M$. Together with Claim 3 we conclude that in Case (i) the payoff to player $j$ does not decrease by going from $\beta$ to $\widetilde{\beta}$.

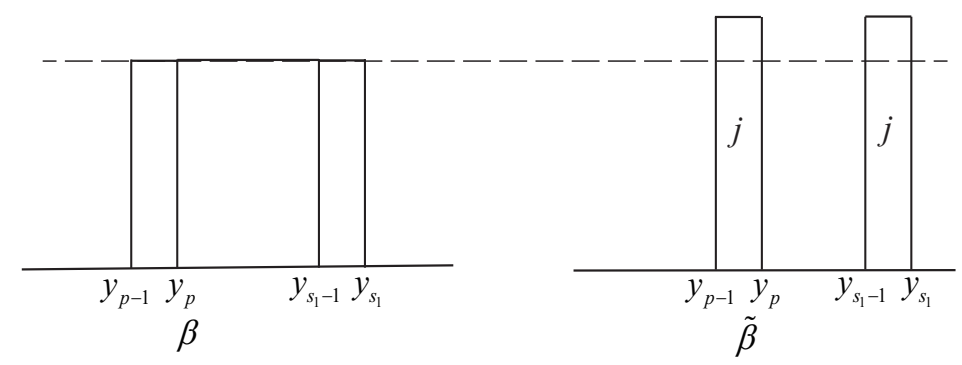

FIGURE 4.10: Illustrating the final argument in Case (i)

Case (ii): $\widetilde{\beta}_{[n]}\left(s_{1}\right) \leq \beta_{[n]}\left(s_{0}\right)-1$.

In this case, by Claim 4 there is again at most one $t^{\prime} \in M, s_{0}<t^{\prime} \leq s_{1}$, such that $\widetilde{\beta}_{[n]}\left(t^{\prime}\right)=\beta_{[n]}\left(t^{\prime}\right)+1$. If $t^{\prime} \neq s_{1}$, then $\widetilde{\beta}_{[n]}\left(s_{1}\right)=\beta_{[n]}\left(s_{1}\right) \leq \beta_{[n]}\left(t^{\prime}\right)=$ 
$\widetilde{\beta}_{[n]}\left(t^{\prime}\right)-1$. Now, by Claim 3 , when going from $\beta$ to $\widetilde{\beta}$, player $j$ has a gain of

$$
\frac{1}{\widetilde{\beta}_{[n]}\left(s_{1}\right)},
$$

and a loss of at most

$$
\frac{1}{\beta_{[n]}\left(s_{0}\right)}+\frac{1}{\beta_{[n]}\left(t^{\prime}\right)}-\frac{1}{\widetilde{\beta}_{[n]}\left(t^{\prime}\right)} .
$$

We have

$$
\begin{aligned}
\frac{1}{\widetilde{\beta}_{[n]}\left(s_{1}\right)}-\frac{1}{\beta_{[n]}\left(s_{0}\right)} & \geq \frac{1}{\widetilde{\beta}_{[n]}\left(s_{1}\right)}-\frac{1}{\widetilde{\beta}_{[n]}\left(s_{1}\right)+1} \\
& \geq \frac{1}{\widetilde{\beta}_{[n]}\left(t^{\prime}\right)-1}-\frac{1}{\widetilde{\beta}_{[n]}\left(t^{\prime}\right)} \\
& =\frac{1}{\beta_{[n]}\left(t^{\prime}\right)}-\frac{1}{\widetilde{\beta}_{[n]}\left(t^{\prime}\right)},
\end{aligned}
$$

hence player $j$ 's gain is at least as large as the maximal loss. Again, together with Claim 3, it follows that also in Case (ii) player j's payoff, when going from $\beta$ to $\widetilde{\beta}$, cannot decrease.

By repeating the argument of part (b), by (**) we end up with the profile resulting from player $j$ playing the $1 \mathrm{rm}-\hat{r}$ action, while in each step player $j$ 's payoff cannot decrease. With $(*)$, this completes the proof of the theorem. 



\section{Chapter 5}

\section{Core selections for transferable utility games}

\subsection{Introduction}

The core of a cooperative game with transferable utility is initially introduced by Gillies [27]; it is a set that allocates the grand coalitional worth and ensures that each coalition obtains at least what it could gain on its own. In other words, once the players receive a core allocation, no coalition has any incentive to deviate from the grand coalition. The core has gained vast popularity and various fields including economics, political science, and operations research have successfully employed this concept. As a consequence, our attention is focused on the core of a transferable utility game.

Regardless of the benefits that the core presents, problems may occur to the players in a game, when it comes to multiple points of the core. Even though different agents may wish to select distinct allocations in the core, all players tend to be rational. More specifically, the allocations, which are certain to minimize some players' profits, will not be accepted by these players. Therefore, such allocations should be deleted to encourage the whole group

Adapted from: 'Kong Q. and Sun H. and Xu G. and Hou D.: Associated games to optimize the core of a transferable utility game. Journal of Optimization Theory and Applications 182: 816-836, 2019'. 
to stick together, which also benefits the forecast for the final selection. Alternatively, it is necessary to sharpen the core to an increasingly justified set by invoking extra criteria. Ideally, we can select a unique point from the core in a game and consider it as the final allocation. In other words, the narrowing of the core under some reasonable criteria can be seen as the optimization of the core. This chapter mainly concentrates on the core optimization of a transferable utility game.

Of course, the interest in the optimization of the core is not new. The $\varepsilon$-core is formally treated for the first time in Shapley and Shubik [62], who define it by imposing a uniform bonus to coalition formation. Geometrically, the $\varepsilon$-core is obtained by "pushing in" all boundaries of the core named hyperplanes with equidistant movement. Furthermore, the least core, proposed by Maschler et al. [44], is defined as the intersection of all nonempty $\varepsilon$-cores. Now, the specific bonus is the maximum such that the corresponding $\varepsilon$-core is nonempty. Given that the pushes of all hyperplanes can be performed nonuniformly, another optimization of the core called the weak $\varepsilon$-core is also presented in Shapley and Shubik [62] by imposing a per-capita bonus to coalition formation. The main difference among these approaches above lies in the way coalitions are subsidized. For each coalition, the bonus it earns depends solely on its characteristics, such as the total worth or the size. By contrast, a bonus system based on every coalition's power or ability comes closer to the actual reward mechanisms, such as the salary paid.

According to each coalition's power or ability, this chapter presents a new perspective towards the optimization of the core by changing the original game. A new associated game ${ }^{1}$ is employed to be an adaptation of a given game such that it reflects an optimistic self-assessment of every coalition's worth. A statement on our imaginary associated game is given as follows: A coalition may believe that the appropriation of at least a part of the surplus, generated by its cooperation with the whole of its complement, is within reach. In consequence, every coalition is more willing to compute its payment

\footnotetext{
${ }^{1}$ Hamiache [30] gives an interpretation for his associated game. A coalition may believe that the appropriation of at least a part of the surpluses, generated by its cooperation with each one of the isolated players in its complementary coalition, is within reach. As a consequence, this coalition may revaluate its own worth as the summation of its worth in the original game and of a uniform percentage of all the possible previous surpluses.
} 
based on the summation of its worth in the original game and of a varying percentage of the surplus. Here, the varying percentage, which enables us to adjust the worth of a coalition to "greater" or "lesser" degree with different proportion, is the corresponding coalition's weight indicating its ability or power. It should be noted that our associated game is a generalized form of the Sh-complement-associated game in Hwang et al. [35]. More accurately, our associated game is more reasonable in real applications than the Sh-complement-associated game. To better optimize the core of a transferable utility game, we recursively define a sequence of associated games. This recursive process terminates, when there exists a game with an empty core. Otherwise, we can generate an infinite sequence of associated games whose cores are all nonempty. Subsequently, the major problem is to determine if the sequence of associated games converges.

The algebraic representation and matrix approach are adopted to address this problem. Xu et al. [69] introduce the coalitional-matrix and state that the Shapley value and the associated game can be presented algebraically by their coalitional matrices. In this chapter, the sequence of associated games can also be represented algebraically by the associated transformation matrix and the original game. Through the diagonal decomposition of the associated transformation matrix, we prove that the infinite sequence of associated games converges and that the limit game is a constant-sum one. Now, the core of the limit game, a single point, is regarded as the final optimized set of the core. Actually, in order to obtain the final point from the core, we only need to calculate the eigenvalues and the eigenvectors of the transformation matrix, which makes the computations much easier compared with other outcomes in the core, such as the nucleolus in Schmeidler [57], the fairest core in Nguyen [46], or the core-center in González-Díaz and SánchezRodríguez [28]. In addition, the matrix approach to determine the limit game has advantages over the method used by Hwang et al. [35], which fails to explore the limit game of the sequence of our associated games. The proposed matrix approach can be applied to investigate the limit game of the sequence of Sh-complement-associated games, which is specified in Section 5.5.

This chapter is organized as follows: In Section 5.2, we recall necessary 
definitions of cooperative games. The associated games are presented to optimize the core in Section 5.3. The matrix approach is applied to deal with the limit game of the infinite sequence of our associated games in Section 5.4. Section 5.5 presents the concrete forms of the limit games with some special weight coefficients. In Section 5.6, a numerical example is illustrated. Section 5.7 concludes with a brief summary.

\subsection{Preliminaries}

A cooperative game with transferable utility (TU) is a pair $(N, v)$, where $N$ is a finite set of $n$ players and $v: 2^{N} \rightarrow \mathbb{R}$ is a mapping from the power set of $N$ to the real number set $\mathbb{R}$. It is always assumed that $v(\emptyset)=0$. A subset of $N$ is called a coalition. For each coalition $S \subseteq N, v(S)$ denotes the worth that coalition $S$ achieves with its members cooperating altogether. We use $|S|$ to denote the cardinality of coalition $S$. The set of all TU games with player set $N$ is denoted by $G^{N} . \mathbb{N}_{+}$stands for the positive integer set and $\subset$ denotes the proper subset.

For any game $(N, v) \in G^{N}$, its dual game $\left(N, v^{*}\right)$ is defined as $v^{*}(S)=$ $v(N)-v(N \backslash S)$ for any $S \subseteq N$. If there is no ambiguity, we identify the game $(N, v)$ with its characteristic function $v$ and the dual game $\left(N, v^{*}\right)$ with $v^{*}$. Clearly, it holds that $\left(v^{*}\right)^{*}=v$, i.e., the duality of the dual game agrees with the original game. A game $v$ is said to be constant-sum, if $v(S)+v(N \backslash S)=$ $v(N)$ for all $S \subseteq N$. It is easy to get that $v=v^{*}$, if and only if a game $v$ is constant-sum.

In this context, an allocation is a vector in $\mathbb{R}^{n}$, the $n$-dimensional Euclidean space, whose coordinates are indexed by the players. Given an allocation $x=$ $\left(x_{1}, x_{2}, \cdots, x_{n}\right), x(S)=\sum_{i \in S} x_{i}$ denotes the total payment to its members of coalition $S \subseteq N$. For convenience, we simply write $x_{i}$ instead of $x(\{i\})$ for each $i \in N$. An allocation $x \in \mathbb{R}^{n}$ is said to be efficient or a preimputation, if $x(N)=v(N)$. An imputation is a preimputation such that $x_{i} \geq v(i)$ for any $i \in N$. $I^{*}(N, v)$ and $I(N, v)$ denote the preimputation and imputation sets, respectively. A value $\varphi: G^{N} \rightarrow \mathbb{R}^{n}$ is a mapping that assigns a vector $\varphi(N, v) \in \mathbb{R}^{n}$ to every game $(N, v) \in G^{N}$. 
Let $(N, v) \in G^{N}$. The core $c(N, v)$, introduced by Gillies [27], is the set of all preimputations where every coalition receives at least the worth it can obtain by cooperating altogether. Formally,

$$
c(N, v)=\left\{x \in I^{*}(N, v): x(S) \geq v(S) \text { for all } S \subset N\right\} .
$$

As a consequence, no coalition wants to deviate from the grand coalition, once the players receive a core allocation.

Let $e^{v}(S, x)=v(S)-x(S)$ denote the excess function, which is taken as a measure of the discontentment of coalition $S$ towards a given allocation $x$ in a game $v$. In addition, the greater $e^{v}(S, x)$ is, the more ill-treated $S$ would feel. Consequently, the core can also be given by

$$
c(N, v)=\left\{x \in I^{*}(N, v): e^{v}(S, x) \leq 0 \text { for all } S \subset N\right\} .
$$

In other words, the core can be viewed as the efficient set in which all coalitions' excesses are nonpositive.

Let $v \in G^{N}, x \in I(N, v)$ and $y \in I(N, v)$. It is said that $x$ dominates $y$ (notation: $x \succ_{D}^{S} y$ ), if there exists a nonempty coalition $S$ such that $x_{i}>y_{i}$ for all $i \in S$ and $\sum_{i \in S} x_{i} \leq v(S)$. Consider an imputation $y$ proposed as an outcome for the game. If some coalition $S$ can improve upon $y$, in order to obtain a higher aggregate payoff, it may threaten to leave the grand coalition and thereby suggest an alternative imputation $x$ to replace $y$.

When it comes to why it may be reasonable to presume that an element in the core would eventually replace the initial allocation outside the core, Sengupta and Sengupta [59] propose a new relation between imputations named indirect domination. They say that $x$ indirectly dominates $y$ (in symbols, $x \succ_{I} y$ ), if there exists a finite sequence of imputations $\left\{y^{1}, y^{2}, \cdots, y^{m}\right\}$ and a finite collection of coalitions $\left\{S_{0}, \cdots, S_{m}\right\}$ such that $y^{1} \succ_{D}^{S_{0}} y ; y^{j} \succ_{D}^{S_{j-1}} y^{j-1}$ for $j=2,3, \cdots, m ; x \succ_{D}^{S_{m}} y^{m}$. Moreover, they show that given any allocation outside the core, an allocation in the core indirectly dominates it.

Recall that the core of a TU game is the set of efficient allocations where each coalition obtains at least what it could gain on its own. As the objective is to optimize the core of a TU game, agents can elaborate some expectations 
of the original game and be more willing to compute their payments based on these expectations. More specifically, an imaginary game should be raised to revaluate each coalition's worth, which emphasizes the problem of putting forward the imaginary game in a fair way.

\subsection{Associated games to optimize the core}

To begin with, we list an example to illustrate that not all allocations in the core are accepted by all players. Consider a cooperative game with $N=$ $\{1,2,3\}$. The worths of all coalitions are given in Table 5.1.

TABLE 5.1 The worths of all coalitions.

\begin{tabular}{cccccccc}
\hline$S$ & 1 & 2 & 3 & 12 & 13 & 23 & $N$ \\
\hline$v(S)$ & 54 & 82 & 58 & 150 & 106 & 132 & 210 \\
\hline
\end{tabular}

It can be observed that there are many allocations in the core. However, not all allocations are desirable for players to pursue the maximal benefit or minimize the complaint (dissatisfaction). Consider a core point $(70,82,58)$. Since no welfare is generated through the cooperation, player 2 will refuse to accept the payment of 82 , which is equal to what it could gain on its own. Similarly, so does player 3 . Then players 2 and 3 tend to deviate from the grand coalition, which leads to an interrupted cooperation. As a result, player 1 can only obtain 54 by acting alone. Economically, there can be as many allocations as those in the core that may not be accepted by some of the three players besides the given allocation $(70,82,58)$. Consequently, the imaginary game should be defined to sharpen the core to a more reasonable subset and ideally to a single point by removing the unsatisfactory allocations for the three players. Before we introduce our imaginary associated game, the Shcomplement-associated game in Hwang et al. [35] is primarily presented.

Definition 5.1. (Hwang et al. [35]) Let $(N, v) \in G^{N}$. The Sh-complementassociated game $\left(N, v_{1}^{S h}\right)$ is defined by specifying, for all $S \subseteq N$,

$$
v_{1}^{S h}(S)=v(S)+\lambda\left(v^{*}(S)-v(S)\right) .
$$


Practically, each coalition should earn more worth according to its own ability or power. Therefore, our associated game is defined as follows.

Definition 5.2. Given any game $(N, v) \in G^{N}$ and any coefficient $\lambda(S), S \subseteq N$ in the $(0,1)$ normalization, the associated game $v_{1}$ is given by

$$
v_{1}(S)=v(S)+\lambda(S)\left(v^{*}(S)-v(S)\right) \quad \text { for all } S \subseteq N .
$$

Here, $\lambda(S)$ is the weight of the coalition $S \subseteq N$, which enables us to adjust the worth of the coalition $S$ to greater or lesser degree with different ratio. This is a major difference from the Sh-complement-associated game. Moreover, the greater the weight is, the more worth the corresponding coalition can obtain. Note that $v_{1}(\emptyset)=0$ and $v_{1}(N)=v(N)$.

The dual game of $v_{1}$ is explicitly generated as

$$
v_{1}^{*}(S)=\lambda(N \backslash S) v(S)+(1-\lambda(N \backslash S)) v^{*}(S) \text { for all } S \subseteq N .
$$

It is again immediate that $v_{1}^{*}(\emptyset)=0$ and $v_{1}^{*}(N)=v(N)$.

Remark 5.1. (i) As for $\lambda(S)=\lambda$ for all $S \subseteq N$ with $\lambda$ being a constant, the associated game $v_{1}$ is the "constant mollifier" as defined by Charnes et al. [10] or the Sh-complement-associated game.

(ii) Concerning $\lambda(S)=\lambda_{|S|}$ for all $S \subseteq N$, the associated game $v_{1}$ is the "coalitional mollifier" in Charnes et al. [10], where $\lambda_{|S|}$ is a variable only depending on the size of the coalition $S$.

The following proposition is necessary to develop a further relationship between the cores of our associated game and the original game, when both cores are nonempty. Charnes et al. [10] show that the core of a game can be characterized alternatively using the duality in an upper bound form.

Proposition 5.1. (Charnes et al. [10]) For any game $(N, v) \in G^{N}$ with the nonempty core, the following two conditions are equivalent with respect to a preimputation $x$.

(i) $x \in c(N, v)$. 
(ii) $v(S) \leq x(S) \leq v^{*}(S)$ for all $S \subseteq N$.

This proposition indicates that the core of a game $(N, v)$ can be equivalently depicted as

$$
c(N, v)=\left\{x \in I^{*}(N, v): v(S) \leq x(S) \leq v^{*}(S) \text { for all } S \subseteq N\right\},
$$

in which $v^{*}(S)$ can be considered as a maximum feasible bound of the coalition $S$ and $v(S)$ as a minimum feasible one. Specifically, for any allocation in the core, all coalitions can assure themselves of the worths in the original game and have great expectations for targeting the maximum. More interestingly, our imaginary associated game lies between the original game and its dual game as a convex combination. Moreover, the duality of such an associated game is also between the original game and its dual game. Thus, the core of our associated game is included in the core of the original game.

Theorem 5.1. Given a game $(N, v) \in G^{N}$ with the nonempty core and its associated game $\left(N, v_{1}\right)$, it holds that $c\left(N, v_{1}\right) \subseteq c(N, v)$, when the set $c\left(N, v_{1}\right)$ is nonempty.

Proof. As both $v_{1}$ and $v_{1}^{*}$ can be regarded as a convex combination of $v$ and $v^{*}$, it yields that for all $S \subseteq N$,

$$
v(S) \leq v_{1}(S) \leq v^{*}(S) \text { and } v(S) \leq v_{1}^{*}(S) \leq v^{*}(S) .
$$

Furthermore, by Proposition 5.1, it is true that for any $x \in c\left(N, v_{1}\right)$,

$$
v(S) \leq v_{1}(S) \leq x(S) \leq v_{1}^{*}(S) \leq v^{*}(S) \text { for any } S \subseteq N .
$$

As a result, it is deduced that $c\left(N, v_{1}\right) \subseteq c(N, v)$.

Subsequently, we show that given any allocation outside the core $c\left(N, v_{1}\right)$, there is an allocation in $c\left(N, v_{1}\right)$ that indirectly dominates it.

Theorem 5.2. Let $(N, v)$ be a game in $G^{N}$ and let $\left(N, v_{1}\right)$ be the associated game with the nonempty core. For each feasible allocation $x \notin c\left(N, v_{1}\right)$, there exists $y \in c\left(N, v_{1}\right)$ such that $y \succ_{I} x$. 
Proof. Let $x^{k} \notin c\left(N, v_{1}\right), k \in \mathbb{N}_{+} \cup\{0\}$ with $x^{0}=x$. Choose a set $S_{k}$ such that $e^{v_{1}}\left(S_{k}, x^{k}\right) \geq e^{\nu_{1}}\left(S, x^{k}\right)$ for all $S \subset N$, it is true that $e^{\nu_{1}}\left(S_{k}, x^{k}\right)>0$. Now, select an arbitrary allocation $z \in c\left(N, v_{1}\right)$. We can identify, for each $k \in \mathbb{N}_{+} \cup\{0\}$, the set $T_{k}$ satisfying $T_{k}=\left\{i \in S_{k}: x_{i}^{k}<z_{i}\right\}$. Thus, it holds that $T_{k} \neq \emptyset$. Furthermore, there exists at least one $i_{k}^{*} \in N \backslash S_{k}$ such that $x_{i_{k}^{*}}^{k} \neq z_{i_{k}^{*}}$.

Define

$$
x_{i}^{k+1}= \begin{cases}x_{i}^{k}+\frac{e^{v_{1}}\left(S_{k}, x^{k}\right)}{\left|T_{k}\right|}, & \text { if } i \in T_{k}, \\ x_{i}^{k}, & \text { if } i \in S_{k} \backslash T_{k}, \\ z_{i}, & \text { if } i \in N \backslash S_{k} \text { and } i \neq i_{k}^{*}, \\ z_{i}+v(N)-v_{1}\left(S_{k}\right)-z\left(N \backslash S_{k}\right), & \text { if } i=i_{k}^{*},\end{cases}
$$

where $\left|T_{k}\right|$ is the cardinality of $T_{k}$. According to Sengupta and Sengupta [59], the construction indeed leads, in finitely many steps, to an allocation $y=x^{k^{*}} \in c\left(N, v_{1}\right)$.

Using the combination of Theorem 5.1 and Theorem 5.2, we can say that the set $c\left(N, v_{1}\right)$ is more stable than $c(N, v)$, since some coalitions can improve upon the prevailing proposal in $c(N, v) \backslash c\left(N, v_{1}\right)$ by an individually rational and efficient counterproposal at each intermediate step.

Now, let us consider two examples to illustrate the geometric features of $c(N, v)$ and $c\left(N, v_{1}\right)$. For the given game in Table 5.1, six hyperplanes of the form $H_{S}^{v}=\left\{x \in I^{*}(N, v): x(S)=v(S)\right\}, \emptyset \neq S \subset N$ and the core $c(N, v)$ are firstly shown in Fig. 5.1, where the hyperplanes and the core are represented as the solid lines and the bold solid trapezoid, respectively. Next, we push in all the hyperplanes $H_{S}^{v}, \emptyset \neq S \subset N$. The core $c\left(N, v_{1}\right)$ is obtained, when the push of a hyperplane $H_{S}^{v}$ is the amount of $\lambda(S)\left(v^{*}(S)-v(S)\right)$ for any $\emptyset \neq S \subset N$. In view of $\lambda(1)=\frac{1}{3}, \lambda(2)=\lambda(3)=\lambda(12)=\lambda(13)=\frac{1}{4}$ and $\lambda(23)=\frac{1}{2}$, all the hyperplanes $H_{S}^{v_{1}}, \emptyset \neq S \subset N$ and the core $c\left(N, v_{1}\right)=\{x \in$ $\left.I^{*}(N, v): 62 \leq x_{1} \leq 66,87.5 \leq x_{2} \leq 98.5,58.5 \leq x_{3} \leq 59.5\right\}$ are then generated, as shown by the broken lines and the bold broken trapezoid in Fig. 5.1, respectively.

Consider $\lambda(1)=\lambda(2)=\lambda(3)=\frac{1}{4}$ and $\lambda(12)=\lambda(13)=\lambda(23)=$ $\frac{1}{2}$. Fig. 5.2 shows the cores $c(N, v)$ and $c\left(N, v_{1}\right)$, which are represented as the bold solid and broken trapezoids, respectively. The hyperplanes $H_{S}^{v}$ 
and $H_{S}^{\nu_{1}}, \emptyset \neq S \subset N$ are also described in Fig. 5.2, the solid and broken lines, respectively. Additionally, we derive that $v_{1}(1)=60, v_{1}(2)=87.5$, $v_{1}(3)=58.5, v_{1}(12)=151, v_{1}(13)=117, v_{1}(23)=144$, and $c\left(N, v_{1}\right)=$ $\left\{x \in I^{*}(N, v): 60 \leq x_{1} \leq 66,87.5 \leq x_{2} \leq 93,58.5 \leq x_{3} \leq 59\right\}$. As the geometric features of $c(N, v)$ and $c\left(N, v_{1}\right)$ are similar to those in Fig. 5.1, we will not elaborate here.

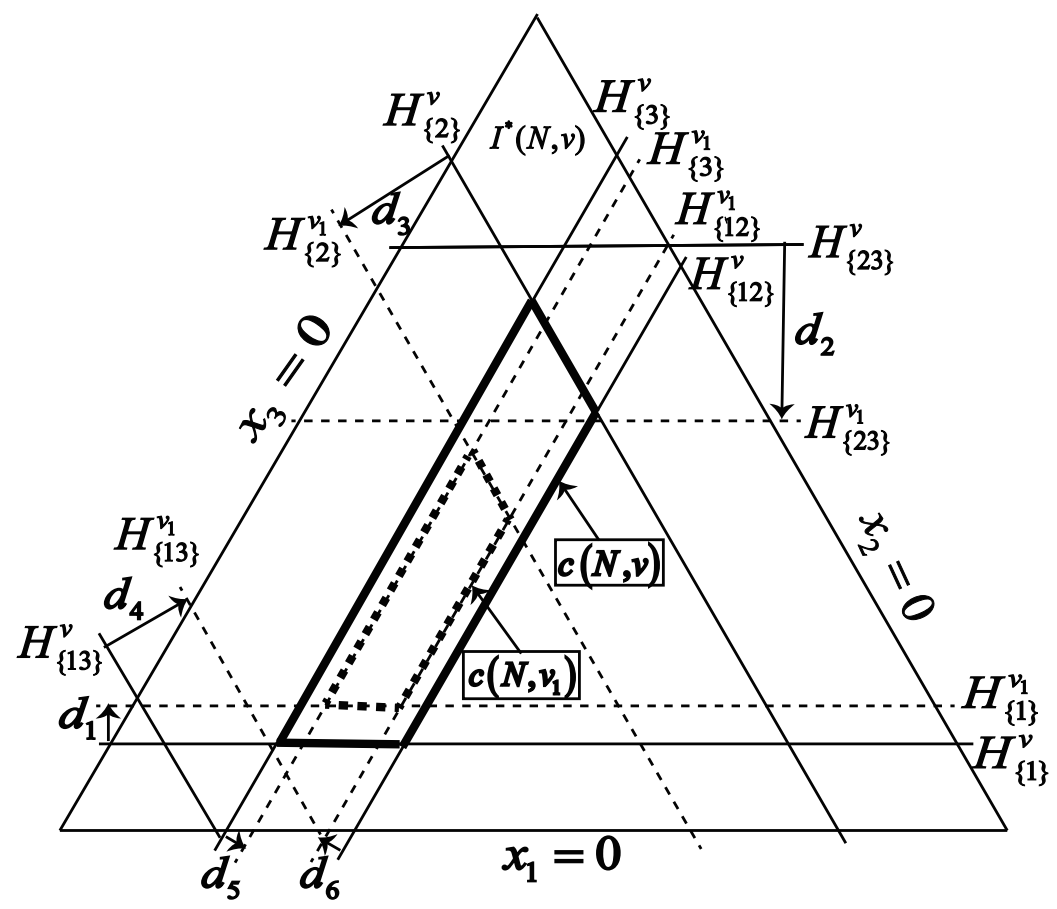

FIGURE 5.1: Geometrical features of $c(N, v)$ and $c\left(N, v_{1}\right)$ with $\lambda(1)=\frac{1}{3}, \lambda(2)=\lambda(3)=\lambda(12)=$ $\lambda(13)=\frac{1}{4}, \lambda(23)=\frac{1}{2}$, where $d_{1}=\frac{1}{3}\left(v^{*}(1)-v(1)\right)$, $d_{2}=\frac{1}{4}\left(v^{*}(23)-v(23)\right), \quad d_{3}=\frac{1}{4}\left(v^{*}(2)-v(2)\right)$, $d_{4}=\frac{1}{4}\left(v^{*}(13)-v(13)\right), \quad d_{5}=\frac{1}{4}\left(v^{*}(3)-v(3)\right)$, $d_{6}=\frac{1}{2}\left(v^{*}(12)-v(12)\right)$.

In order to improve the optimization of the core of a TU game, a $k$ associated game is recursively defined starting from $k-1$ for any $k \in \mathbb{N}_{+}$ with $v_{0}=v$.

Definition 5.3. For any game $(N, v) \in G^{N}$ and any weight coefficient $\lambda(S)$, 


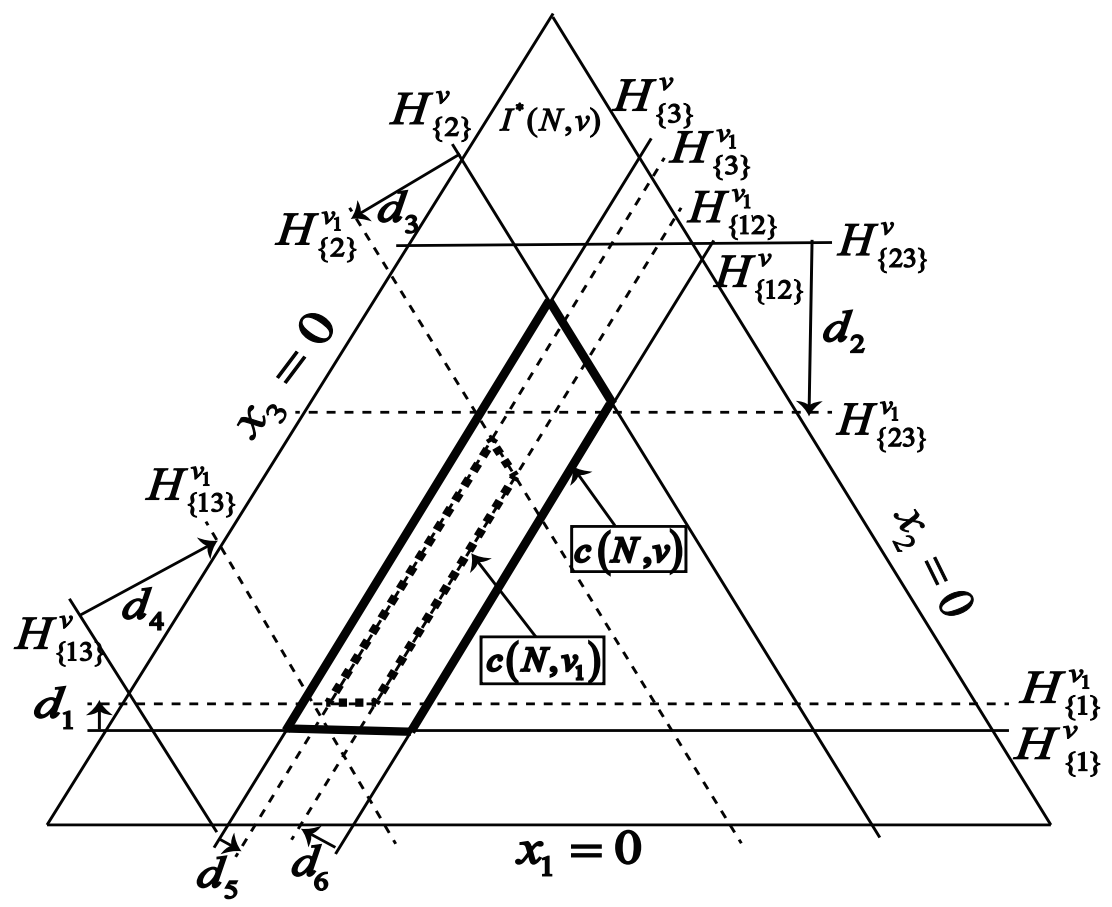

FIGURE 5.2: Geometrical features of $c(N, v)$ and $c\left(N, v_{1}\right)$ with $\lambda(1)=\lambda(2)=\lambda(3)=\frac{1}{4}, \lambda(12)=\lambda(13)=\lambda(23)=$ $\frac{1}{2}$, where $d_{1}=\frac{1}{4}\left(v^{*}(1)-v(1)\right), d_{2}=\frac{1}{4}\left(v^{*}(23)-v(23)\right)$, $d_{3}=\frac{1}{4}\left(v^{*}(2)-v(2)\right), d_{4}=\frac{1}{2}\left(v^{*}(13)-v(13)\right), d_{5}=$ $\frac{1}{2}\left(v^{*}(3)-v(3)\right), d_{6}=\frac{1}{2}\left(v^{*}(12)-v(12)\right)$.

$S \subseteq N$ in the $(0,1)$ normalization, the $k$-associated game $v_{k}, k \in \mathbb{N}_{+}$is inductively given by

$$
v_{k}(S):=(1-\lambda(S)) v_{k-1}(S)+\lambda(S) v_{k-1}^{*}(S) \quad \text { for all } S \subseteq N,
$$

where $v_{0}:=v$ and $v_{0}^{*}:=v^{*}$.

Therefore, the dual game $v_{k}^{*}$ of the $k$-associated game $v_{k}, k \in \mathbb{N}_{+}$is obtained as

$$
v_{k}^{*}(S)=\lambda(N \backslash S) v_{k-1}(S)+(1-\lambda(N \backslash S)) v_{k-1}^{*}(S) \text { for all } S \subseteq N,
$$


where $v_{0}=v$ and $v_{0}^{*}=v^{*}$. Then we can also conclude that $c\left(N, v_{k}\right)$ is more stable than $c\left(N, v_{k-1}\right)$ for any $k \in \mathbb{N}_{+}$with $v_{0}=v$.

Theorem 5.3. Let $(N, v)$ be a game in $G^{N}$ and let $v_{k}, k \in \mathbb{N}_{+}$be the $k$-associated game with the nonempty core.

(i) For any $k \in \mathbb{N}_{+}$, it holds that $c\left(N, v_{k}\right) \subseteq c\left(N, v_{k-1}\right)$, where $v_{0}=v$.

(ii) For any $x \notin c\left(N, v_{k}\right)$, there exists $y \in c\left(N, v_{k}\right)$ such that $y \succ_{I} x$.

As a result, the attention is narrowed down to the set $c\left(N, v_{k}\right)$. For a more intuitive description of the sequence of associated games, Figs. 5.3 and 5.4 are presented: Case 1 shows that the recursive process is terminated, when there exists an integer $l \in \mathbb{N}_{+}$such that $c\left(N, v_{l}\right) \neq \emptyset$ and $c\left(N, v_{l+1}\right)=\emptyset$. At this time, the nonempty core $c\left(N, v_{l}\right)$ is the final optimized set of the core of the original game. Back to the game in Table 5.1, all coalitions' worths over the games $v_{1}$ and $v_{2}$ are listed in Table 5.2, when $\lambda(1)=\frac{1}{3}, \lambda(2)=$ $\lambda(3)=\lambda(12)=\lambda(13)=\frac{1}{4}, \lambda(23)=\frac{1}{2}$. Since we verify that $c\left(N, v_{2}\right)=\emptyset$ and $c\left(N, v_{1}\right) \neq \emptyset$, the final optimized set is $c\left(N, v_{1}\right)=\left\{x \in I^{*}(N, v): 62 \leq\right.$ $\left.x_{1} \leq 66,87.5 \leq x_{2} \leq 98.5,58.5 \leq x_{3} \leq 59.5\right\}$, as shown by the bold broken trapezoid in Fig. 5.1.

TABLE 5.2 All coalitions' worths over the games $v_{1}$ and $v_{2}$.

\begin{tabular}{cccccccc}
\hline$S$ & 1 & 2 & 3 & 12 & 13 & 23 & $N$ \\
\hline$v_{1}(S)$ & 62 & 87.5 & 58.5 & 150.5 & 111.5 & 144 & 210 \\
\hline$v_{2}(S)$ & $\frac{190}{3}$ & 90.25 & 58.75 & 150.75 & 114.25 & 146 & 210 \\
\hline
\end{tabular}

Otherwise, in case 2, we can obtain an infinite sequence of associated games $\left\{\left(N, v_{k}\right)\right\}_{k=0}^{\infty}$ whose cores are all nonempty, where $v_{0}=v$. This case happens to the game in Table 5.1, when $\lambda(1)=\lambda(2)=\lambda(3)=\frac{1}{4}$ and $\lambda(12)=\lambda(13)=\lambda(23)=\frac{1}{2}$. Henceforth, the major problem is to determine if the infinite sequence of associated games converges. In the next section, the algebraic representation and matrix approach are adopted. 


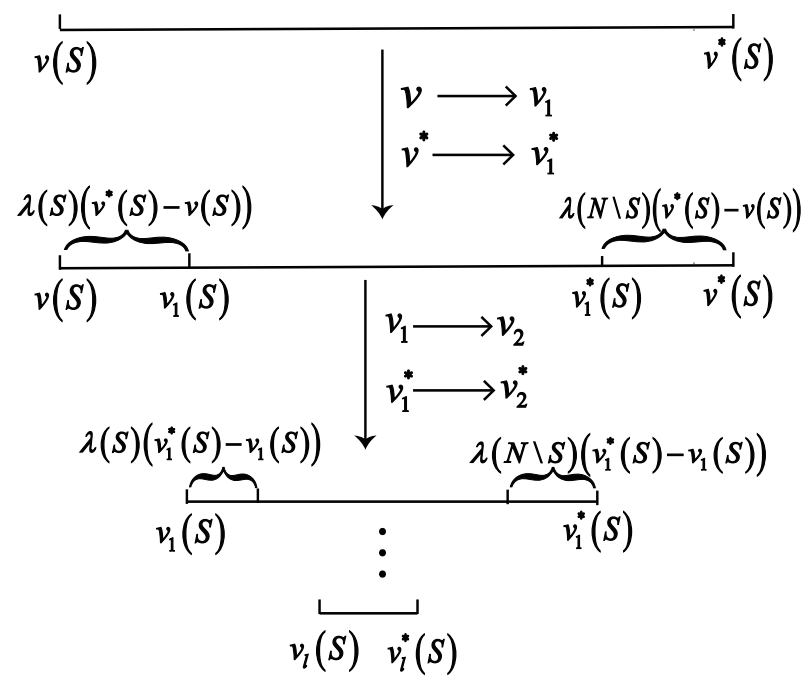

Case 1: $c\left(N, v_{l}\right) \neq \varnothing, c\left(N, v_{l+1}\right)=\varnothing, l \in \mathbb{N}_{+}$

FIGURE 5.3: The recursive process of the sequence of associated games.

\subsection{Matrix approach to select one outcome from the core}

It is known that every game $v$ can always be identified by its column vector of the worths of all coalitions in the lexicographic order ${ }^{2}$. Formally,

$$
\mathbf{v}=(v(S))_{\emptyset \neq S \subseteq N}=(v(1), \ldots, v(n), v(12), \ldots, v(N))^{T} .
$$

In a sense, the vector space $\mathbb{R}^{2^{n}-1}$ is isomorphic to the set $G^{N}$ of all $n$-person cooperative games.

Similarly, a method that uses a basis, such as the unanimity game basis, of the $2^{n}-1$ dimensional vector/game space is much older. Shapley [60] constructs the unanimity game to characterize the Shapley value. Dragan $[14,15]$

\footnotetext{
${ }^{2}$ Coalition $S=\left\{i_{1}, i_{2}, \cdots, i_{s}\right\}$ with $i_{1}<i_{2}<\cdots<i_{s}$ precedes coalition $T=\left\{j_{1}, j_{2}, \cdots, j_{t}\right\}$ with $j_{1}<j_{2}<\cdots<j_{t}$, if and only if the sizes of these two coalitions verify either $s<t$, or $s=t$ and for some $k, 1 \leq k<s$, it holds that $i_{l}=j_{l}$ for $1 \leq l<k$ and $i_{k}<j_{k}$.
} 


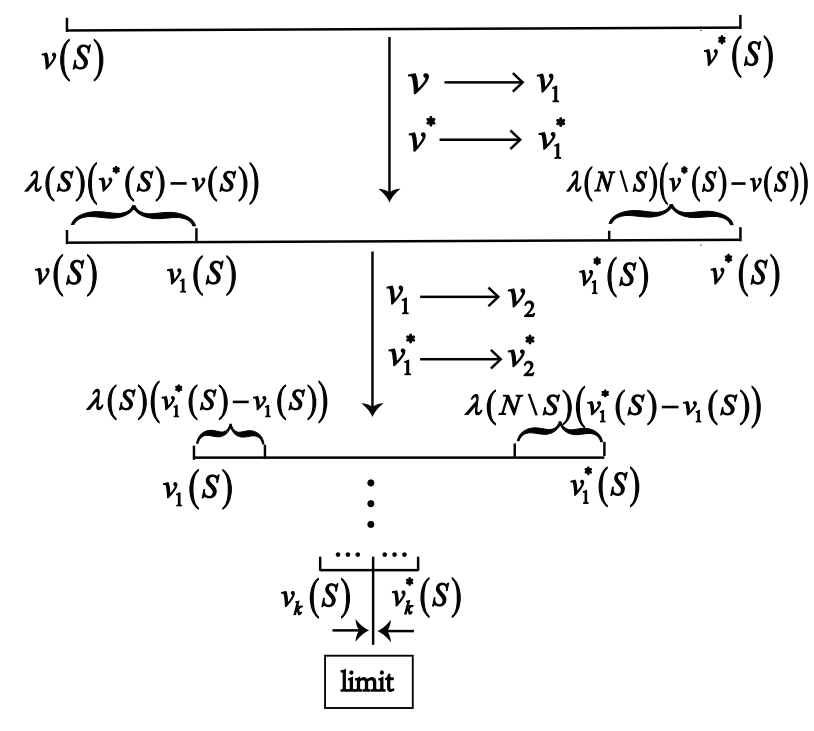

Case 2: $c\left(N, v_{k}\right) \neq \varnothing, k \in \mathbb{N}_{+}$

FIGURE 5.4: The recursive process of the sequence of associated games.

introduces the potential basis to study the weighted Shapley value and the Banzhaf value. Yokote et al. [72] present a new basis to characterize the Shapley value. The matrix approach is also a natural and powerful technique for research in the field of cooperative game theory. Following this matrix approach, well-known results as well as new theorems and characterizations of a certain class of some values are derived. The matrix approach has been applied to study associated game consistency for the Shapley value by $\mathrm{Xu}$ et al. [69, 70] and Hamiache [31]. Hamiache [32] also uses this approach to study the efficient Aumann-Drèze value for TU games with coalition structures and the efficient Myerson value ${ }^{3}$ for TU games with communication structures. This approach is also applied by Xu et al. [71] to study the Center of gravity of Imputation Set (CIS) value and the Egalitarian Non-Separable

\footnotetext{
${ }^{3}$ The efficient Aumann-Drèze value is a normalization of the Aumann-Drèze value via weighted Shapley value (see Kalai and Samet [36]). The efficient Myerson value is a normalization of the Myerson value via the same weighted Shapley value. As expected, they verify the efficiency property: Given a game $(N, v) \in G^{N}$, a value $\varphi \in \mathbb{R}^{n}$ is efficient, if $\sum_{i \in N} \varphi_{i}=v(N)$.
} 
Contribution (ENSC) value in Driessen and Funaki [16].

$\mathrm{Xu}$ et al. [69] call a matrix $\mathrm{M}$ row (respectively, column)-coalitional, if its rows (respectively, columns) are indexed by all lexicographically ordered coalitions. $\mathbf{M}$ is called square-coalitional, if it is both row-coalitional and column-coalitional. Then the following results are shown to explore the limit game of the sequence of associated games in their approach.

(i) For any linear operator that maps a game $v$ to its associated game $v_{1}$ for some $\lambda$, and the corresponding infinite sequence of associated games $\left\{\left(N, v_{k}\right)\right\}_{k=1}^{\infty}$, there exists an associated transformation matrix $\mathbf{M}_{\lambda}$ such that $\mathbf{v}_{\mathbf{1}}=\mathbf{M}_{\lambda} \cdot \mathbf{v}$ and $\mathbf{v}_{\mathbf{k}}=\left(\mathbf{M}_{\lambda}\right)^{k} \cdot \mathbf{v}, k \in \mathbb{N}_{+}$.

(ii) Using the diagonal decomposition $\mathbf{M}_{\lambda}=\mathbf{P D}_{\lambda} \mathbf{P}^{-1}$, where $\mathbf{D}_{\lambda}$ is the diagonal matrix with all diagonal elements being the eigenvalues of $\mathbf{M}_{\lambda}$ and $\mathbf{P}$ is the matrix consisting of all corresponding eigenvectors, it follows that $\left(\mathbf{M}_{\lambda}\right)^{k}=\mathbf{P}\left(\mathbf{D}_{\lambda}\right)^{k} \mathbf{P}^{-\mathbf{1}}$. By the restrictions on $\lambda$, the limit game of the sequence of associated games exists and is determined as

$$
\tilde{\mathbf{v}}=\lim _{k \rightarrow \infty} \mathbf{v}_{\mathbf{k}}=\lim _{k \rightarrow \infty}\left(\mathbf{M}_{\lambda}\right)^{k} \cdot \mathbf{v}=\mathbf{P} \lim _{k \rightarrow \infty}\left(\mathbf{D}_{\lambda}\right)^{k} \mathbf{P}^{-\mathbf{1}} \cdot \mathbf{v}=\mathbf{P D P}^{-\mathbf{1}} \cdot \mathbf{v} .
$$

In what follows, we apply this approach to determine the limit game of the sequence of our associated games. Our associated game $\mathbf{v}_{\mathbf{1}}$ can be expressed algebraically by the associated transformation matrix $\mathbf{M}_{\lambda(S)}$ and the original game $\mathbf{v}$, that is, $\mathbf{v}_{\mathbf{1}}=\mathbf{M}_{\lambda(S)} \cdot \mathbf{v}$, where $\mathbf{M}_{\lambda(S)}$ under the normalized weights $\lambda(S), \emptyset \neq S \subset N$ is both row-coalitional and column-coalitional, defined by

$$
\mathbf{M}_{\lambda(S)}=\left(\begin{array}{ccccc}
1-\lambda(1) & \cdots & 0 & -\lambda(1) & \lambda(1) \\
\vdots & \ddots & \vdots & \vdots & \vdots \\
-\lambda(N \backslash 1) & \cdots & 0 & 1-\lambda(N \backslash 1) & \lambda(N \backslash 1) \\
0 & \cdots & 0 & 0 & 1
\end{array}\right)_{\left(2^{n}-1\right) \times\left(2^{n}-1\right)}
$$

Henceforth, the sequences of our associated games $\left\{\left(N, \mathbf{v}_{\mathbf{k}}\right)\right\}_{k=0}^{\infty}$, dual games $\left\{\left(N, \mathbf{v}_{\mathbf{k}}^{*}\right)\right\}_{k=0}^{\infty}$ and cores $\left\{c\left(N, \mathbf{v}_{\mathbf{k}}\right)\right\}_{k=0}^{\infty}$ can be alternatively expressed 
by

$$
\begin{aligned}
& \mathbf{v}_{\mathbf{k}}=\mathbf{M}_{\lambda(S)} \cdot \mathbf{v}_{\mathbf{k}-\mathbf{1}}=\cdots=\left(\mathbf{M}_{\lambda(S)}\right)^{k} \cdot \mathbf{v}, \\
& \mathbf{v}_{\mathbf{k}}^{*}=\mathbf{M}_{\mathbf{1}} \cdot \mathbf{v}_{\mathbf{k}}=\mathbf{M}_{\mathbf{1}}\left(\mathbf{M}_{\lambda(S)}\right)^{k} \cdot \mathbf{v}, \\
& c\left(N, \mathbf{v}_{\mathbf{k}}\right)=\left\{x \in I^{*}(N, v): \mathbf{x} \geq \mathbf{v}_{\mathbf{k}}\right\},
\end{aligned}
$$

where $\mathbf{v}_{\mathbf{0}}=\mathbf{v}, \mathbf{v}_{\mathbf{0}}^{*}=\mathbf{v}^{*}, \mathbf{M}_{\mathbf{1}}=\left(\begin{array}{cccc}0 & \cdots & -1 & 1 \\ \vdots & \ddots & \vdots & \vdots \\ -1 & \cdots & 0 & 1 \\ 0 & \cdots & 0 & 1\end{array}\right)$ and $\mathbf{x}$ is identified by its column vector of the payments of all lexicographically ordered coalitions.

Inspired by Proposition 5.1, the core $c\left(N, \mathbf{v}_{\mathbf{k}}\right), k \in \mathbb{N}_{+}$with respect to $x \in I^{*}(N, v)$ can be equivalently stated as

$$
c\left(N, \mathbf{v}_{\mathbf{k}}\right)=\left\{x \in I^{*}(N, v): \mathbf{v}_{\mathbf{k}} \leq \mathbf{x} \leq \mathbf{v}_{\mathbf{k}}^{*}\right\} .
$$

Now, we recall some results in algebra theory for deriving more properties of the associated transformation matrix.

Proposition 5.2. (Xu et al. [69]) Let $\mathbf{M}$ be a square matrix of order $l$.

(i) The dimension $d$ of the solution space of the linear system of equations $\mathbf{M x}=\mathbf{0}$ satisfies $d=l-\operatorname{rank}(\mathbf{M})$.

(ii) For every eigenvalue of matrix $\mathbf{M}$, its (algebraic) multiplicity is at least the dimension of the corresponding eigenspace.

(iii) The sum of the multiplicities of all eigenvalues of matrix $\mathbf{M}$ equals $l$.

(iv) The matrix $\mathbf{M}$ is diagonalizable, if and only if the sum of the dimensions of the distinct eigenspaces equals $l$, and this happens, iff the dimension of the eigenspace for each eigenvalue equals the multiplicity of the eigenvalue.

The main purpose of Theorem 5.4 is to determine the eigenvalues and the eigenvectors of the associated transformation matrix $\mathbf{M}_{\lambda(s)}$ under the normalized weights $\lambda(S), \emptyset \neq S \subset N$ by its characteristic polynomial. 
Theorem 5.4. $1,1-\lambda(1)-\lambda(N \backslash 1), 1-\lambda(2)-\lambda(N \backslash 2), \cdots, 1-\lambda(S)-$ $\lambda(N \backslash S)$, where $S$ is the $\left(2^{n-1}-1\right)^{t h}$ of all lexicographically ordered coalitions, are the eigenvalues of associated transformation matrix $\mathbf{M}_{\lambda(S)}$ under the normalized weights $\lambda(S), \emptyset \neq S \subset N$, and the multiplicities corresponding to these eigenvalues are $2^{n-1}, 1,1, \cdots, 1$.

Proof. Let $\mathbf{E}$ be the identity matrix. The characteristic polynomial of matrix $\mathbf{M}_{\lambda(S)}$ is

$$
\begin{aligned}
& \left|a \mathbf{E}-\mathbf{M}_{\lambda(S)}\right|=\left|\begin{array}{ccccc}
a-1+\lambda(1) & 0 & \cdots & \lambda(1) & -\lambda(1) \\
0 & a-1+\lambda(2) & \cdots & 0 & -\lambda(2) \\
\vdots & \vdots & \ddots & \vdots & \vdots \\
\lambda(N \backslash 1) & 0 & \cdots & a-1+\lambda(N \backslash 1) & -\lambda(N \backslash 1) \\
0 & 0 & \cdots & 0 & a-1
\end{array}\right| \\
& =-\left|\begin{array}{ccccc}
a-1+\lambda(1) & 0 & \cdots & \lambda(1) & -\lambda(1) \\
\lambda(N \backslash 1) & 0 & \cdots & a-1+\lambda(N \backslash 1) & -\lambda(N \backslash 1) \\
\vdots & \vdots & \ddots & \vdots & \vdots \\
0 & a-1+\lambda(2) & \cdots & 0 & -\lambda(2) \\
0 & 0 & \cdots & 0 & a-1
\end{array}\right| \\
& =\left|\begin{array}{ccccc}
a-1+\lambda(1) & \lambda(1) & \cdots & 0 & -\lambda(1) \\
\lambda(N \backslash 1) & a-1+\lambda(N \backslash 1) & \cdots & 0 & -\lambda(N \backslash 1) \\
\vdots & \vdots & \ddots & \vdots & \vdots \\
0 & 0 & \cdots & a-1+\lambda(2) & -\lambda(2) \\
0 & 0 & \cdots & 0 & a-1
\end{array}\right| \\
& =(a-1)^{3}(a-1+\lambda(1)+\lambda(N \backslash 1))(a-1+\lambda(2)+\lambda(N \backslash 2)) . \\
& \left|\begin{array}{ccc}
a-1+\lambda(3) & & \lambda(3) \\
& \ddots & \\
\lambda(N \backslash 3) & & a-1+\lambda(N \backslash 3)
\end{array}\right| \\
& =(a-1)^{2^{n-1}}(a-1+\lambda(1)+\lambda(N \backslash 1)) \cdots(a-1+\lambda(S)+\lambda(N \backslash S)),
\end{aligned}
$$

where $S$ is the $\left(2^{n-1}-1\right)^{t h}$ of all lexicographically ordered coalitions. The second equality is due to the exchange between the second and $\left(2^{n}-2\right)^{\text {th }}$ rows of $\left|a \mathbf{E}-\mathbf{M}_{\lambda(s)}\right|$, and the third one is owing to the exchange of the second 
and $\left(2^{n}-2\right)^{t h}$ columns of $\left|a \mathbf{E}-\mathbf{M}_{\lambda(s)}\right|$. The omitted process is the repetition of the similar exchanges until the final characteristic polynomial is obtained. Thus, the eigenvalues of the associated transformation matrix $\mathbf{M}_{\lambda(S)}$ under the normalized weights $\lambda(S), \emptyset \neq S \subset N$ and the multiplicities corresponding to these eigenvalues are straightforward to generate.

Motivated by Proposition 5.2 and Theorem 5.4, we derive that the associated matrix $\mathbf{M}_{\lambda(S)}$ under the normalized weights $\lambda(S), \emptyset \neq S \subset N$ can be diagonalizable. There are some important properties of the associated transformation matrix $\mathbf{M}_{\lambda(S)}$ under the normalized weights $\lambda(S), \emptyset \neq S \subset N$ presented by its diagonalization.

Proposition 5.3. Given the associated transformation matrix $\mathbf{M}_{\lambda(S)}$ with $0<$ $\lambda(S)<1$ for any $\emptyset \neq S \subset N$, the following results are presented.

(i) It is deduced that $\mathbf{M}_{\lambda(s)}=\mathbf{P D}_{\lambda(s)} \mathbf{P}^{-1}$, where

$\mathbf{D}_{\lambda(S)}=\operatorname{diag}(\underbrace{1,1, \cdots, 1}_{2^{n-1} \text { times }}, 1-\lambda(1)-\lambda(N \backslash 1), \cdots, 1-\lambda(S)-\lambda(N \backslash S))$

and $\mathbf{P}$ consists of the eigenvectors of matrix $\mathbf{M}_{\lambda(S)}$ corresponding to eigenvalues $1,1-\lambda(1)-\lambda(N \backslash 1), 1-\lambda(2)-\lambda(N \backslash 2), \cdots, 1-\lambda(S)-$ $\lambda(N \backslash S)$ with $S$ as the $\left(2^{n-1}-1\right)^{t h}$ of all lexicographically ordered coalitions.

(ii) We conclude that $\lim _{k \rightarrow \infty}\left(\mathbf{M}_{\lambda(S)}\right)^{k}=\mathbf{P D P}^{-\mathbf{1}}$, where

$$
\mathbf{D}=\operatorname{diag}(\underbrace{1,1, \cdots, 1}_{2^{n-1} \text { times }}, \underbrace{0,0, \cdots, 0}_{2^{n-1}-1 \text { times }}) \text {. }
$$

Proof. Part (i) is directly inferred by Theorem 5.4. Part (ii) holds that

$$
\begin{aligned}
\lim _{k \rightarrow \infty}\left(\mathbf{M}_{\lambda(S)}\right)^{k} & =\lim _{k \rightarrow \infty}\left(\mathbf{P D}_{\lambda(S)} \mathbf{P}^{-1}\right)^{k}=\lim _{k \rightarrow \infty} \mathbf{P}\left(\mathbf{D}_{\lambda(s)}\right)^{k} \mathbf{P}^{-\mathbf{1}} \\
& =\mathbf{P} \operatorname{diag}(\underbrace{1,1, \cdots, 1}_{2^{n-1} \text { times }}, \underbrace{0,0, \cdots, 0}_{2^{n-1}-1 \text { times }}) \mathbf{P}^{-\mathbf{1}}
\end{aligned}
$$




$$
=\mathrm{PDP}^{-1} \text {, }
$$

where the fourth equality is due to $0<\lambda(S)<1$ for any $\emptyset \neq S \subset N$.

Using Proposition 5.3, we originate the next theorem regarding the convergences of the sequences of associated games and dual games.

Theorem 5.5. Given the sequences of associated games $\left\{\left(N, \mathbf{v}_{\mathbf{k}}\right)\right\}_{k=0}^{\infty}$ and dual games $\left\{\left(N, \mathbf{v}_{\mathbf{k}}^{*}\right)\right\}_{k=0}^{\infty}$, we can deduce the following.

(i) The limit game $(N, \tilde{\mathbf{v}})$ of the sequence of associated games $\left\{\left(N, \mathbf{v}_{\mathbf{k}}\right)\right\}_{k=0}^{\infty}$ is given by

$$
\tilde{\mathbf{v}}=\mathbf{P D P}^{-1} \cdot \mathbf{v}
$$

(ii) The sequence of dual games $\left\{\left(N, \mathbf{v}_{\mathbf{k}}^{*}\right)\right\}_{k=0}^{\infty}$ converges to the game $\left(N, \tilde{\mathbf{v}}^{*}\right)$, where

$$
\tilde{\mathbf{v}}^{*}=\mathbf{M}_{1} \mathbf{P D P}^{-1} \cdot \mathbf{v}
$$

Thus far, we have proved that the sequence of associated (dual) games converges. As a consequence, the recursive process can be terminated, which also solves the problem in the last paragraph of Section 5.3. Interestingly, we can prove that the limit game $(N, \tilde{v})$ is a constant-sum one.

Theorem 5.6. Let $\left\{\left(N, \mathbf{v}_{\mathbf{k}}\right)\right\}_{k=0}^{\infty}$ be the sequence of associated games. The limit game $(N, \tilde{v})$ is a constant-sum one.

Proof. Firstly, we show $\tilde{v}=\tilde{v}^{*}$ to prove that $(N, \tilde{v})$ is a constant-sum game. For the sake of convenience, let $\mathbf{D}=\operatorname{diag}(1,0,1,0, \cdots, 1)$. Analytically, the matrix $\mathbf{M}_{1} \mathbf{P D}$ is equal to the matrix $\mathbf{P D}$, i.e.,

$$
M_{1} P D=\left(y_{1}, 0, y_{2}, 0, \cdots, y_{2^{n-1}-1}, 0, y_{2^{n-1}}\right)=P D,
$$

where the column-vectors $\mathbf{y}_{1}, \mathbf{y}_{2}, \cdots, \mathbf{y}_{2^{\mathrm{n}-1}-1}, \mathbf{y}_{2^{\mathrm{n}-1}}$ are the different eigenvectors of $\mathbf{M}_{\lambda(S)}$ corresponding to the eigenvalue 1 , and $\mathbf{0}$ denotes a zero column-vector. Since both sides of Equation (5.4) multiplied by $\mathbf{P}^{-1}$ will not affect the relation of equivalence, it holds that

$$
\mathrm{M}_{1} \mathrm{PDP}^{-1}=\mathrm{PDP}^{-1} .
$$


Based on Equations (5.2) and (5.3), it is true that $\tilde{\mathbf{v}}=\tilde{\mathbf{v}}^{*}$.

In addition, an equivalent state for $\tilde{\mathbf{v}}=\tilde{\mathbf{v}}^{*}$ yields that for every $S \subseteq N$,

$$
\tilde{v}(S)=\tilde{v}^{*}(S)=\tilde{v}(N)-\tilde{v}(N \backslash S) \Leftrightarrow \tilde{v}(S)+\tilde{v}(N \backslash S)=\tilde{v}(N) .
$$

As a result, the limit game $\tilde{v}$ is a constant-sum one.

Subsequently, the final optimized set of the core is proved to be a single point, when there is no game with an empty core in this sequence.

Theorem 5.7. Let $\left\{\left(N, \mathbf{v}_{\mathbf{k}}\right)\right\}_{k=0}^{\infty}$ be the sequence of associated games whose cores are all nonempty and let $(N, \tilde{v})$ be its limit game. We deduce that

$$
c(N, \tilde{v})=(\tilde{v}(1), \tilde{v}(2), \cdots, \tilde{v}(n)) .
$$

Proof. From Equation (5.1) and Theorem 5.6, we obtain that

$$
c(N, \tilde{v})=\left\{x \in I^{*}(N, \tilde{v}): x(S)=\tilde{v}(S) \text { for all } S \subseteq N\right\},
$$

which eventually leads to $c(N, \tilde{v})=(\tilde{v}(1), \tilde{v}(2), \cdots, \tilde{v}(n))$ because of the non-emptiness of the set $c(N, \tilde{v})$.

\begin{tabular}{|c|c|c|c|c|c|c|c|}
\hline $\mathbf{v}$ & & & $(54,82,58$ & 150,106 & $132,210)^{T}$ & & \\
\hline $\mathbf{P}$ & $\begin{array}{c}0.7071 \\
0 \\
0 \\
0 \\
0 \\
-0.7071 \\
0\end{array}$ & $\begin{array}{c}0.4472 \\
0 \\
0 \\
0 \\
0 \\
0.8944 \\
0\end{array}$ & $\begin{array}{c}0 \\
0 \\
0.7071 \\
-0.7071 \\
0 \\
0 \\
0\end{array}$ & $\begin{array}{c}0 \\
0 \\
0.4472 \\
0.8944 \\
0 \\
0 \\
0\end{array}$ & $\begin{array}{c}0 \\
-0.4472 \\
0 \\
0 \\
-0.8944 \\
0 \\
0\end{array}$ & $\begin{array}{c}-0.7071 \\
0 \\
0 \\
0 \\
0 \\
0.7071 \\
0\end{array}$ & $\left.\begin{array}{l}0.3128 \\
0.2085 \\
0.3128 \\
0.3128 \\
0.4170 \\
0.3128 \\
0.6255\end{array}\right)$ \\
\hline D & \multicolumn{7}{|c|}{$\operatorname{diag}(1,0,1,0,0,1,1)$} \\
\hline$\tilde{\mathbf{v}}=\mathbf{P D P}^{-1} \cdot \mathbf{v}$ & \multicolumn{7}{|c|}{$\left(62, \frac{268}{3}, \frac{176}{3}, \frac{454}{3}, \frac{362}{3}, 148,210\right)^{T}$} \\
\hline$c(N, \tilde{v})$ & \multicolumn{7}{|c|}{$\left(62, \frac{268}{3}, \frac{176}{3}\right)$} \\
\hline
\end{tabular}

TABLE 5.3 The related variables under $\lambda(1)=\lambda(2)=$ $\lambda(3)=\frac{1}{4}$ and $\lambda(12)=\lambda(13)=\lambda(23)=\frac{1}{2}$.

Finally, let us optimize the core of the given game in Table 5.1, when $\lambda(1)=\lambda(2)=\lambda(3)=\frac{1}{4}$ and $\lambda(12)=\lambda(13)=\lambda(23)=\frac{1}{2}$. The related variables are described in Table 5.3, and the final selection is $c(N, \tilde{v})=$ 
$\left(62, \frac{268}{3}, \frac{176}{3}\right)$.

\subsection{Special cases of weight coefficients}

In this section, we will focus on the special weight coefficients of $\lambda(S)=\lambda_{|S|}$, $S \subseteq N$ denoting that the weight of a coalition is only related to its cardinality, and of $\lambda(S)=\lambda, S \subseteq N$ standing for a constant that does not depend on any coalition. Particularly, we give the concrete forms of the limit games of these two cases.

Theorem 5.8. For the associated transformation matrix under the two special cases above, we provide the following results.

(i) Let $\lambda(S)=\lambda_{|S|}$ for any $S \subseteq N$. The limit game is $\tilde{\mathbf{v}}=\mathbf{v}_{\mathbf{1}}$, whenever $\lambda_{|S|}+\lambda_{n-|S|}=1$ for any $1 \leq|S|<n$.

(ii) When it holds that $\lambda(S)=\lambda$ for any $S \subseteq N$, the limit game is presented as

$$
\tilde{\mathbf{v}}=\left(\begin{array}{cccc}
\frac{1}{2} & \cdots & -\frac{1}{2} & \frac{1}{2} \\
\vdots & \ddots & \vdots & \vdots \\
-\frac{1}{2} & \cdots & \frac{1}{2} & \frac{1}{2} \\
0 & \cdots & 0 & 1
\end{array}\right) \cdot \mathbf{v} .
$$

Proof. Point (i): In view of $\lambda_{|S|}+\lambda_{n-|S|}=1$ for any $1 \leq|S|<n$, we derive that

$$
\begin{aligned}
\mathbf{M}_{1} \cdot \mathbf{M}_{\lambda_{|S|}} & =\left(\begin{array}{cccc}
0 & \cdots & -1 & 1 \\
\vdots & \ddots & \vdots & \vdots \\
-1 & \cdots & 0 & 1 \\
0 & \cdots & 0 & 1
\end{array}\right)\left(\begin{array}{ccccc}
1-\lambda_{1} & \cdots & -\lambda_{1} & \lambda_{1} \\
\vdots & \ddots & \vdots & \vdots \\
-\lambda_{n-1} & \cdots & 1-\lambda_{n-1} & \lambda_{n-1} \\
0 & \cdots & 0 & 1
\end{array}\right) \\
& =\left(\begin{array}{cccc}
1-\lambda_{1} & \cdots & -\lambda_{1} & \lambda_{1} \\
\vdots & \ddots & \vdots & \vdots \\
-\lambda_{n-1} & \cdots & 1-\lambda_{n-1} & \lambda_{n-1} \\
0 & \cdots & 0 & 1
\end{array}\right)=\mathbf{M}_{\lambda_{|S|},}
\end{aligned}
$$


equivalent to $\mathbf{v}_{\mathbf{1}}^{*}=\mathbf{v}_{\mathbf{1}}$. Furthermore, the definitions of associated games yield that

$$
\tilde{\mathbf{v}}=\lim _{k \rightarrow \infty} \mathbf{v}_{\mathbf{k}}=\mathbf{v}_{\mathbf{1}}=\mathbf{v}_{\mathbf{1}}^{*} .
$$

Point (ii): According to Equation (5.4), the matrix PD equals

$$
\mathrm{PD}=\left(\mathrm{y}_{1}, 0, \mathrm{y}_{2}, \mathbf{0}, \cdots, \mathrm{y}_{2^{\mathrm{n}-1}-1}, 0, \mathrm{y}_{2^{\mathrm{n}-1}}\right),
$$

where the column-vectors $\mathbf{y}_{1}, \mathbf{y}_{2}, \cdots, \mathbf{y}_{2^{n-1}-1}, \mathbf{y}_{2^{n-1}}$ are the different eigenvectors of $\mathbf{M}_{\lambda(S)}$ corresponding to the eigenvalue 1 , and $\mathbf{0}$ denotes a zero column-vector.

Concerning $\lambda(S)=\lambda$ for any $S \subseteq N$, we derive that

$$
\mathbf{y}_{1}=\left(\begin{array}{c}
\frac{\sqrt{2}}{2} \\
0 \\
\vdots \\
0 \\
-\frac{\sqrt{2}}{2} \\
0
\end{array}\right), \cdots, \mathbf{y}_{2^{\mathrm{n}-1}-1}=\left(\begin{array}{c}
0 \\
-\frac{\sqrt{2}}{2} \\
\vdots \\
\frac{\sqrt{2}}{2} \\
0 \\
0
\end{array}\right), \mathbf{y}_{2^{\mathrm{n}-1}}=\left(\begin{array}{c}
y_{1} \\
y_{2} \\
\vdots \\
y_{2^{n}-3} \\
y_{2^{n}-2} \\
y_{2^{n}-1}
\end{array}\right)
$$

where $y_{1}, y_{2}, \cdots, y_{2^{n-1}} \in \mathbb{R}$.

Furthermore, we generate that

$$
\mathbf{P}^{-1}=\left(\mathbf{z}_{1}, \mathbf{z}_{1}^{\prime}, \mathbf{z}_{2}, \mathbf{z}_{2}^{\prime} \cdots, \mathbf{z}_{2^{\mathrm{n}-1}-1}, \mathbf{z}_{2^{\mathrm{n}-1}-1}, \mathbf{z}_{2^{\mathrm{n}-1}}\right),
$$

where

$$
\mathbf{z}_{1}=\left(\begin{array}{c}
\frac{\sqrt{2}}{2} \\
\frac{\sqrt{2}}{2} \\
\vdots \\
0 \\
0 \\
0
\end{array}\right), \cdots, \mathbf{z}_{2^{\mathrm{n}-1}-1}=\left(\begin{array}{c}
0 \\
0 \\
\vdots \\
\frac{\sqrt{2}}{2} \\
\frac{\sqrt{2}}{2} \\
0
\end{array}\right), \mathbf{z}_{2^{\mathrm{n}-1}}=\left(\begin{array}{c}
z_{1} \\
z_{2} \\
\vdots \\
z_{2^{n}-3} \\
z_{2^{n}-2} \\
z_{2^{n}-1}
\end{array}\right),
$$


$z_{1}, z_{2}, \cdots, z_{2^{n}-1} \in \mathbb{R}$ and $\mathbf{z}_{1}^{\prime}, \mathbf{z}_{2}^{\prime}, \cdots, \mathbf{z}_{2^{\mathrm{n}-1}-1}^{\prime}$ are the different column-vectors. Note that $y_{2^{n-1}} \cdot z_{2^{n-1}}=1$. Other forms are independent of the final result, so there are no specific expressions. Thus, Equation (5.5) is obtained according to Equation (5.2).

Specifically, Equation (5.5) is equivalent to $\tilde{v}(S)=\frac{1}{2} v(S)+\frac{1}{2} v^{*}(S)$ for all $S \subseteq N$, which is also explored by Hwang et al. [35] with another method. However, the matrix approach has some advantages over their method in determining the limit game. Clearly, the matrix approach can be applied to deal with the limit game of the sequence of Sh-complement-associated games, but their method fails to settle the limit game of the sequence of our associated games. More precisely, the recursive relationships of coefficients $\gamma_{k}, \alpha_{k}$ and $\beta_{k}{ }^{4}$ for the sequence of our associated games cannot be deduced with their method, since it is not true that

$$
v_{k}(N \backslash S) \neq \gamma_{k} v(N \backslash S)+\alpha_{k} v(N)+\beta_{k} v(S), \quad \text { for any } S \subseteq N .
$$

As a result, their method cannot be used to explore the limit game of the sequence of our associated games.

\subsection{A numerical illustration}

Consider a production economic situation in which one boss and two workers are involved, which has already been studied in Shapley and Shubik [63] and in Chetty et al. [11]. It is supposed that the workers have nothing to contribute but their labour and are thought to be of the same type. The boss hires the workers to produce a commodity. For this economic situation containing one boss and two workers, we regard the boss as player 1 and the two workers as player 2 and player 3 . Then this situation can be modelled as

\footnotetext{
${ }^{4}$ In order to investigate the expression of the limit game, they point out that $v_{k}^{S h}(S)$ can be expressed as a linear combination of $v(S), v(N)$ and $v(N \backslash S)$, that is,

$$
v_{k}^{S h}(S)=\gamma_{k} v(S)+\alpha_{k} v(N)+\beta_{k} v(N \backslash S),
$$

where $\gamma_{k}, \alpha_{k}, \beta_{k} \in \mathbb{R}$.
} 
a 3-person cooperative game and the worth of any coalition is described in Table 5.4.

TABLE 5.4 All coalitions' worths over the production economic situation.

\begin{tabular}{lccccccc}
\hline$S$ & 1 & 2 & 3 & 12 & 13 & 23 & $N$ \\
\hline$v(S)$ & 0 & 0 & 0 & 0 & 0 & 0 & 100 \\
\hline
\end{tabular}

Obviously, the definition of the core yields that the allocations of $(100,0,0)$, $(0,100,0)$ and $(0,0,100)$ all belong to the core of the game in Table 5.4. However, the allocation $(100,0,0)$ will not be accepted by the two workers, since they both get nothing. The allocation $(0,100,0)$ will be rejected by the boss and worker 2 , and the boss and worker 1 will exclude the allocation $(0,0,100)$ for the same reason. Thus, we need to optimize the core of this production economy case. It is undisputed that the weight of the boss should be larger than that of each worker. As a result, the weights of $\lambda(1)=\frac{1}{6}, \lambda(2)=\lambda(3)=\frac{1}{7}$ are reasonable. Besides, it is rational to think that the weight of a coalition will not decrease with the increase of the cardinality of the coalition in the production economy case. Consequently, we put $\lambda(12)=\lambda(13)=\frac{1}{3}, \lambda(23)=\frac{1}{4}$. The reason why $\lambda(12)$ is larger than $\lambda(23)$ is that the boss is included in the coalition $\{12\}$. After giving the specific weights of the corresponding coalitions, the related variables under the matrix approach are listed in Table 5. It should be noted that the selection of the weights is not unique and one can also select other justified weights.

According to Table 5.5, the final optimized set of the core of the production economy is the allocation (40,30,30). Followed by the market economy, the boss should be better off than the workers and the incomes of the workers should not be too low to prevent their workforce contribution. As a result, the allocation $(40,30,30)$ is acceptable for the boss and the workers. 
TABLE 5.5 The related variables under $\lambda(1)=\frac{1}{6}, \lambda(2)=$ $\lambda(3)=\frac{1}{7}, \lambda(12)=\lambda(13)=\frac{1}{3}$ and $\lambda(23)=\frac{1}{4}$.

\begin{tabular}{|c|c|c|c|c|c|c|c|}
\hline \multirow[t]{2}{*}{$\mathbf{v}$} & \multicolumn{7}{|c|}{$(0,0,0,0,0,0,100)^{T}$} \\
\hline & 0.7071 & 0.5547 & 0 & 0 & 0 & 0 & 0.3094 \\
\hline \multirow{6}{*}{$\mathbf{P}$} & 0 & 0 & 0 & 0 & -0.3939 & -0.7071 & 0.2518 \\
\hline & 0 & 0 & 0.7071 & 0.3939 & 0 & 0 & 0.3888 \\
\hline & 0 & 0 & -0.7071 & 0.9191 & 0 & 0 & 0.2377 \\
\hline & 0 & 0 & 0 & 0 & -0.9191 & 0.7071 & 0.3746 \\
\hline & -0.7071 & 0.8321 & 0 & 0 & 0 & 0 & 0.3170 \\
\hline & 0 & 0 & 0 & 0 & 0 & 0 & $0.6264)$ \\
\hline D & \multicolumn{7}{|c|}{$\operatorname{diag}(1,0,1,0,0,1,1)$} \\
\hline$\tilde{\mathbf{v}}=\mathbf{P D P}^{-1} \cdot \mathbf{v}$ & \multicolumn{7}{|c|}{$(40,30,30,70,70,60,100)^{T}$} \\
\hline$c(N, \tilde{v})$ & \multicolumn{7}{|c|}{$(40,30,30)$} \\
\hline
\end{tabular}

\subsection{Conclusions}

This chapter helps establish a new perspective towards the study of a game with a nonempty core. The optimization of the core is of great importance because there are always plenty of allocations in the core, and this chapter proposes an improved approach. In our opinion, a bonus system based on each coalition's power or ability rather than its characteristics comes closer to the actual reward mechanisms. According to each coalition's power or ability, we define a sequence of associated games to optimize the core of a transferable utility game in this chapter. Moreover, the matrix approach makes the involved computations much easier. We show how our approach can be used to optimize the core in a production economy involving one boss and two workers. Additionally, such environment seems to be a natural place to apply the tools described in this chapter. 



\section{Bibliography}

[1] Alonso-Meijide J.M. and Fiestras-Janeiro M.G. and García-Jurado I.: A new power index for spatial games. In: Modern Mathematical Tools and Techniques in Capturing Complexity 275-285, 2011

[2] Atlamaz M. and Berden C. and Peters H. and Vermeulen D.: Noncooperative solutions for estate division problems. Games and Economic Behavior 73:39-51, 2011

[3] Aumann R. and Maschler M.: Game theoretic analysis of a bankruptcy problem from the Talmud. Journal of Economic Theory 36:195-213, 1985

[4] Banzhaf J.F: Weighted voting doesn't work: A mathematical analysis. Rutgers Law Review 19: 317-343, 1964

[5] Benati S. and Marzetti G.V.: Probabilistic spatial power indexes. Social Choice and Welfare 40: 391-410, 2013

[6] Bertini C. and Freixas J. and Gambarelli G. and Stach I.: Comparing power indices. International Game Theory Review 15: 1340004, 2013

[7] Blockmans T. and Guerry M.A.: Probabilistic Spatial Power Indexes: The Impact of Issue Saliences and Distance Selection 24: 675-697, 2015

[8] Borel E.: La théorie du jeu et les equations integrales a noyau symmetrique. C. R. Acad. Sci.173, 1304-1308. Translated by Savage LJ (1953) The theory of play and integral equations with skew symmetric kernels. Econometrica 21:97-100, 1921 
[9] Braham M. and Holler M.J.: The impossibility of a preference-based power index 17:137-157, 2005

[10] Charnes A. and Rousseau J. and Seiford L.: Complements, mollifiers and the propensity to disrupt. International Journal of Game Theory 7: 37-50, 1978

[11] Chetty V.K. and Dasgupta D. and Raghavan T.E.S.: Power and distribution of profits. Discussion Paper No. 139, Indian Statistical Institute, Delhi Centre, New Delhi, 1976

[12] Coleman J.S.: Control of collectivities and the power of a collectivity to act. In: Lieberman B (ed.) Social choice. Gordon and Breach London, 269-300, 1971

[13] de Frutos M.A.: Coalitional manipulations in a bankruptcy problem. Review of Economic Design 4: 255-272, 1999

[14] Dragan I.: The potential basis and the weighted Shapley value. Libertas Mathematica 11: 139-150, 1991

[15] Dragan I.: New mathematical properties of the Banzhaf value. European Journal of Operational Research 95: 451-463, 1996

[16] Driessen T.S.H. and Funaki Y.: Coincidence of and collinearity between game theoretic solutions. Operations Research Spektrum 13: 15-30, 1991

[17] Dubey P: On the uniqueness of the Shapley value. International Journal of Game Theory 4: 131-139, 1975

[18] Dubey P. and Einy E. and Haimanko O.: Compound voting and the Banzhaf index. Games and Economic Behavior 51: 20-30, 2005

[19] Einy E.: Semivalues of simple games. Mathematics of Operations Research 12: 185-192, 1987

[20] Einy E. and Haimanko O.: Characterization of the Shapley-Shubik power index without the efficiency axiom. Games and Economic Behavior 73: 615-621,2011 
[21] Enelow J.M. and Hinich M.J.: The spatial theory of voting: An introduction. Cambridge University Press, Cambridge, 1984

[22] Enelow J.M. and Hinich M.J.: The spatial theory of voting: An introduction. Cambridge University Press, Cambridge, 1984

[23] Felsenthal D.S. and Machover M.: The measurement of voting power. Edward Elgar, Cheltenham, 1998

[24] Felsenthal D.S. and Machover M.: Voting power measurement: a story of misreinvention. Social choice and welfare 25: 485-506, 2005

[25] Gale D. and Shapley L.S.: College admissions and the stability of marriage. The American Mathematical Monthly, 69: 9-15, 1962

[26] Gambarelli G. and Owen G.: Indirect control of corporations. International Journal of Game Theory 23: 287-302, 1994

[27] Gillies D.B.: Some theorems on $n$-person games. Ph.D. Thesis, Princeton University, Department of Mathematics. Princeton University Press, Princeton, New Jersey, 1953

[28] González-Díaz J. and Sánchez-Rodríguez E.: A natural selection from the core of a TU game: the core-center. International Journal of Game Theory, 36: 27-46, 2007

[29] Grofman B. and Owen G. and Noviello N. and Glazer A.: Stability and centrality of legislative choice in the spatial context. The American Political Science Review 81: 539-553, 1987

[30] Hamiache G.: Associated consistency and Shapley value. International Journal of Game Theory 30: 279-289, 2001

[31] Hamiache G.: A matrix approach to the associated consistency with an application to the Shapley value. International Game Theory Review 12: 175-187, 2010

[32] Hamiache G.: A Matrix Approach to TU Games with Coalition and Communication Structures. Social Choice and Welfare 38: 85-100, 2012 
[33] Hoede C. and Bakker R.R.: A theory of decisional power. Journal of Mathematical Sociology 8: 309-322, 1982

[34] Holler M.J.: Forming coalitions and measuring voting power. Political studies 30: 262-271, 1982

[35] Hwang Y.A. and Julia R. and Ismail R.: Union negotiations: Complement-associated games. Operations Research Letters 45: 126132, 2017

[36] Kalai E. and Samet D.: On weighted Shapley values. International Journal of Game Theory 16: 205-222, 1987

[37] Karos D. and Peters H.: Indirect control and power in mutual control structures. Games and Economic Behavior 92: 150-165, 2015

[38] Karos D. and Peters H.: Effectivity and power. Games and Economic Behavior 108: 363-378, 2018

[39] Klumpp T. and Konrad K.A.: Sequential majoritarian Blotto games. Working Paper, 2018

[40] Holler M.J.: Forming coalitions and measuring voting power. Political studies 30: 262-271, 1982

[41] Laruelle A. and Valenciano F: Voting and collective decision-making. Cambridge University Press, 2011

[42] Martin M. and Nganmeni Z. and Tchantcho B.: The Owen and Shapley spatial power indices: a comparison and a generalization. Mathematical Social Sciences 89: 10-19, 2017

[43] Mercik J. and Stach I.: On Measurement of Control in Corporate Structures. Transactions on Computational Collective Intelligence XXXI 6479, 2018

[44] Maschler M. and Peleg B. and Shapley L.S.: Geometric properties of the kernel, nucleolus, and related solution concepts. Mathematics of Operations Research 4: 303-338, 1979 
[45] Napel S. and Widgrén M.: The possibility of a preference-based power index 17:377-387, 2005

[46] Nguyen T.D.: The fairest core in cooperative games with transferable utilities. Operations Research Letters 43: 34-39, 2015

[47] Nowak A.S.: On an axiomatization of the Banzhaf value without the additivity axiom. International Journal of Game Theory 26: 137-141, 1997

[48] O'Neill B.: A problem of rights arbitration from the Talmud. Mathematical Social Sciences 2:345-371, 1982

[49] Owen G.: Political games. Naval research logistics quarterly 18: 345355,1971

[50] Owen G. and Shapley L.S.: Optimal location of candidates in ideological space. International journal of game theory 18: 339-356, 1989

[51] Pálvölgyi D. and Peters H. and Vermeulen D.: A strategic approach to multiple estate division problems. Games and Economic Behavior 88:135-152, 2014

[52] Peters H. and Schröder M. and Vermeulen D.: Claim Games for estate division problems. Games and Economic Behavior 116:105-115, 2019

[53] Peters H. and Timmer J. and van den Brink R.: Power on digraphs. Operations research and decisions 26: 107-125, 2016

[54] Peters H. and Zarzuelo J.M.: An axiomatic characterization of the Owen-Shapley spatial power index. International Journal of Game Theory 46: $525-545,2017$

[55] Roth A.E. and Sotomayor M.: Two-sided matching. In: Handbook of game theory with economic applications 1: 485-541, 1992

[56] Rusinowska A. and de Swart H.: Generalizing and modifying the HoedeBakker index. In: Theory and Applications of Relational Structures as Knowledge Instruments II 60-88, 2006 
[57] Schmeidler D.: The nucleolus of a characteristic function game. SIAM Journal on applied mathematics 17: 1163-1170, 1969

[58] Schmidtchen D. and Steunenberg B.: On the possibility of a preferencebased power index: The strategic power index revisited. In: Fara R, Leech, Salles M (eds.) Voting Power and Procedures, Essays in Honour of Dan Felsenthal and Mosh Machover, 2014

[59] Sengupta A. and Sengupta K.: A property of the core. Games and Economic Behavior 12: 266-273, 1996

[60] Shapley L.S.: A value for n-person games. Contributions to the Theory of Games II, Princeton University Press, Princeton 2: 307-317, 1953

[61] Shapley L.S. and Shubik M.: A method for evaluating the distribution of power in a committee system. The American Political Science Review 48: 787-792, 1954

[62] Shapley L.S. and Shubik M.: Quasi-cores in a monetary economy with nonconvex preferences. Econometrica: Journal of the Econometric Society 34: 805-827, 1966

[63] Shapley L.S. and Shubik M.: Ownership and the production function. The Quarterly Journal of Economics 81: 88-111, 1967

[64] Shenoy P.P: The Banzhaf power index for political games. Mathematical Social Sciences 2: 299-315, 1982

[65] Straffin Jr P.D.: Power and stability in politics. Chapter 32 in: Handbook of game theory with economic applications, eds 2: 1127-1151, 1994

[66] Thomson W.: Axiomatic and game-theoretic analysis of bankruptcy and taxation problems: an update. Mathematical Social Sciences 74:41-49, 2015

[67] van den Brink R.: The apex power measure for directed networks. Social Choice and Welfare 19: 845-867, 2002 
[68] von Neumann J. and Mogenstern O.: Theory of Games and Economic Behavior. Princeton University Press, Princeton, New Jersey, 1944

[69] Xu G. and Driessen T.S.H. and Sun H.: Matrix analysis for associated consistency in cooperative game theory. Linear Algebra and its Applications 428: 1571-1586, 2008

[70] Xu G. and Driessen T.S.H and Sun H.: Matrix approach to dual similar associated consistency for the Shapley value. Linear Algebra and its Applications 430: 2896-2897, 2009

[71] Xu G. and van den Brink R. and van der Laan G. and Sun H.: Associated consistency characterization of two linear values for TU games by matrix approach. Linear Algebra and its Applications 471: 224-240, 2015

[72] Yokote K. and Funaki Y. and Kamijo Y.: A new basis and the Shapley value. Mathematical Social Sciences 80: 21-24, 2016 



\section{Summary}

Both cooperative and noncooperative games are studied in this thesis. We investigate the power of players in cooperative games depending on the issues at stake, and the positions of players in marriage problems in Chapters 2 and 3 , respectively. Chapter 4 considers resolving the Nash equilibria and subgame perfect equilibria in sequential claim games. Core selection is studied via a sequence of associated games in Chapter 5 .

Closely inspired by the work of Owen and Shapley [50] on spatial games, Chapter 2 studies issue games. Power indices are then defined by weight vectors on the set of issues. Chapter 3 deals with the power indices of matching problems based on two kinds of 'effectivity functions'. The main parts of Chapters 2 and 3 are devoted to axiomatic characterizations of classes of power indices.

In Chapter 4 sequential claim games are considered, in which players sequentially put claims on an estate in a given order. Each part of the estate is then divided proportionally with respect to the number of claims on it. Our main results are, first, that under an additional restriction on strategies, myopic play is a Nash equilibrium; and, second, by including punishments for deviators, myopic play can also be turned into a subgame perfect equilibrium.

Chapter 5 defines a sequence of associated games to optimize the core of a transferable utility game. As the cores of the associated games are increasingly stable, the last one of the nonempty cores in this sequence is the final optimized set. 



\section{Impact of the thesis}

A power index is a tool to measure and compare the power of an individual player in political and economic situations. In a democratic Parliament, one way to assess this is to model Parliament as a simple game. Economic situations as for instance financial and corporate governance structures might be also modelled by simple games. To such a simple game one may then apply a power index, such as Shapley-Shubik index. Another way to assess this is to consider the position of a political party or the issues at stake. Typical examples occur in the area of corporate governance in Karos and Peters [37] and the US legislative process in Karos and Peters [38].

The estate division problem in Chapter 4 is a historic and classic problem - how to distribute the estate among the players based on their entitlements. One of the famous applications is the bankruptcy problem, which can be traced back to a 2000 year old document in Babylonian Talmud. A recent well-known case is the bankruptcy of Lehman Brothers because of the subprime crisis of 2007.

The idea underlying core allocations is to provide incentives for cooperation excluding those unstable outcomes in which some coalitions are able to reach better results for all their members. However, this core concept does not always provide a unique solution for general cooperative games. Therefore, Chapter 5 focuses on core selection by invoking extra reasonable criteria. Applications arise in managerial and public decision making when a bundle of goods has to be allocated among a set of individuals, taking into account the worth or values of the coalitions under different scenarios. 



\section{About the Author}

Qianqian Kong was born on November 6, 1991 in Lankao Country, Kaifeng City, P.R. of China. From 1998 to 2011, Qianqian completed her elementary and senior and junior high school at her hometown.

In 2011, she got the admission offer from Henan Normal University and majored in Applied Mathematics. After four years study as a bachelor, she was selected by Northwestern Polytechnical University for the master study. Two years later, she joined the Master-PhD project and became a PhD student still under the supervision of Professor Hao Sun.

In September 2019, she started her study at the Quantitative Economics department of Maastricht University as a joint PhD student, supervised by Professor Hans Peters. During that time, she was sponsored by the China Scholarship Council. 